University of Louisville

ThinkIR: The University of Louisville's Institutional Repository

Electronic Theses and Dissertations

$12-2011$

\title{
Histone deacetylase inhibition-mediated cytotoxicity of oligodendrocytes.
}

Toros Ali Dincman 1982-

University of Louisville

Follow this and additional works at: https://ir.library.louisville.edu/etd

\section{Recommended Citation}

Dincman, Toros Ali 1982-, "Histone deacetylase inhibition-mediated cytotoxicity of oligodendrocytes."

(2011). Electronic Theses and Dissertations. Paper 351.

https://doi.org/10.18297/etd/351

This Doctoral Dissertation is brought to you for free and open access by ThinkIR: The University of Louisville's Institutional Repository. It has been accepted for inclusion in Electronic Theses and Dissertations by an authorized administrator of ThinkIR: The University of Louisville's Institutional Repository. This title appears here courtesy of the author, who has retained all other copyrights. For more information, please contact thinkir@louisville.edu. 


\title{
HISTONE DEACETYLASE INHIBITION-MEDIATED CYTOTOXICITY OF OLIGODENDROCYTES
}

\author{
By \\ Toros Ali Dincman \\ A.B., University of Chicago, 2004 \\ M.S., University of Louisville, 2009 \\ A Dissertation \\ Submitted to the Faculty of the \\ School of Medicine of the University of Louisville \\ In Partial Fulfillment of the Requirements \\ For the Degree of \\ Doctor of Philosophy \\ Department of Anatomical Sciences and Neurobiology \\ University of Louisville \\ Louisville, Kentucky
}

December 2011 


\title{
HISTONE DEACETYLASE INHIBITION-MEDIATED CYTOTOXICITY OF OLIGODENDROCYTES
}

\author{
By
}

Toros Ali Dincman

A.B., University of Chicago, 2004

M.S., University of Louisville, 2009

\begin{abstract}
A Dissertation Approved on
\end{abstract}
August 23, 2011

by the following Dissertation Committee:

Scott Whittemore, Dissertation Director

Michal Hetman

Richard Benton

Charles Hubscher 


\section{DEDICATION}

This dissertation is dedicated to:

the countless years of sacrifice, inspiration, devotion, and unrelenting support my mother, Lale Toker-Dincman, gave me so my dreams may become reality, the most compassionate, talented physician-to-be and love of my life, Savanna, my loving and patient father, Taskin Dincman, the most loyal man I know and I am proud to call my brother, Tolga Dincman my successful sister-in-law who I am so happy to call family, Holly Dincman my handsome and incredibly gifted nephew, Blake, who will achieve great things, the advisors and educators who have guided me along my chosen path, and all my friends and colleagues that I have made along the way. 


\section{ACKNOWLEDGEMENTS}

I want to first extend my greatest gratitude to one of the hardest working members of the Whittemore lab of whom I will always be indebted to, one of my best friends and colleagues, Jason Beare. I also want to thank Sujata Saraswat and Aruna Vashista whose invaluable advice, companionship, and guidance have made this work a joy to complete. To all current and past members of the Whittemore and Benton labs, I express my sincere appreciation: Nicholas Kuypers, Amanda Pocratsky, Kariena Andres, Russell Howard, Alyssa Roby, Scott Myers, Qilin Cao, Melissa Maddie, Mark Gruenthal, Janelle Fassbender, and William DeVries. I want to thank KSCIRC Core staff members, Christine Nunn, Kim Fentress, Darlene Burke, and Johnny Morehouse for their tireless work. To the following members of the Hetman, Hagg, and Magnuson labs: Scott Smith, Maciej Pietrak, Jenny Zheng, Shiela Arnold, and Eddie Brown, I thank you. To Victoria King and Brian Wattenberg thank you for your patience and support throughout the MD/PhD program. I want to share my extensive appreciation to Michal Hetman and Charles Hubscher for honest guidance and advice for the successful completion of my dissertation. I also want to thank Richard Benton for not only providing great insight into my experimental work but also being a wonderful friend. Finally, thank you to Scott Whittemore whose mentorship has provided me extensive learning, maturation, and instilled a sense of the importance of advancing knowledge of human biological function. 


\section{TABLE OF CONTENTS}

Page

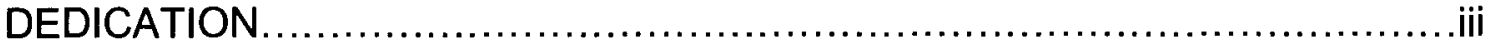

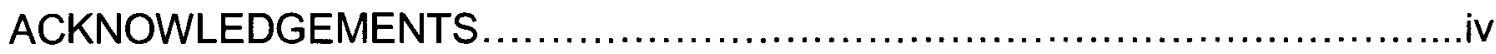

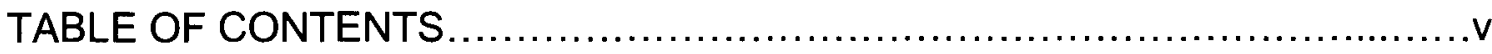

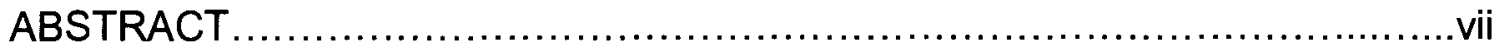

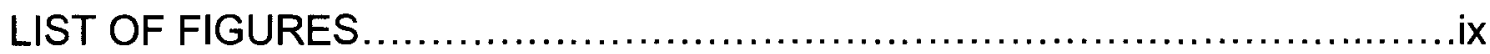

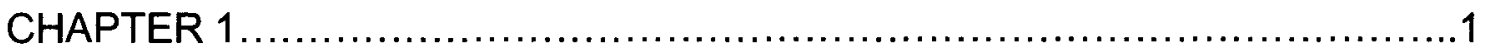

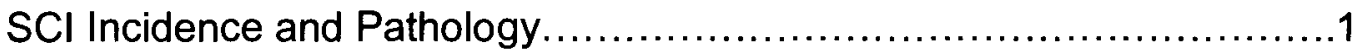

Oligodendrocytes: Relevance and Development.......................

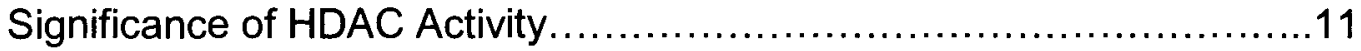

Role of HDACs in DNA Repair..................................... 19

$\mathrm{CICl}$ : Significance and Pathophysiology.............................25

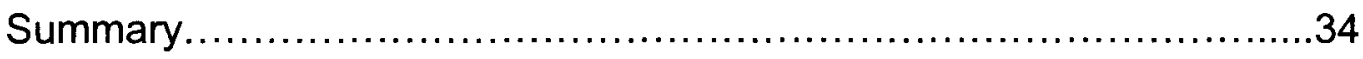

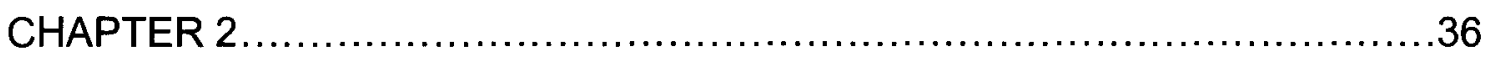

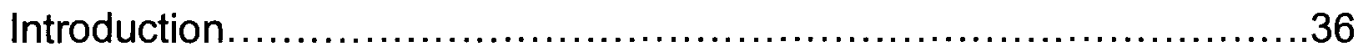

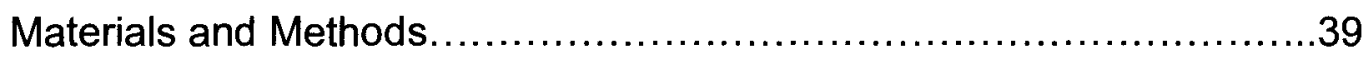

Results............................................................ 46

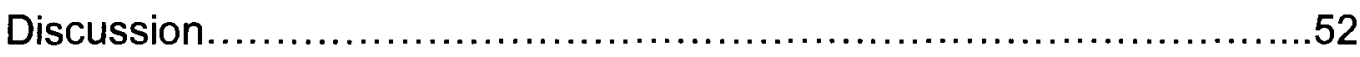

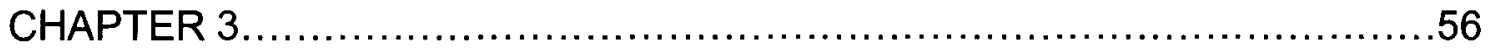

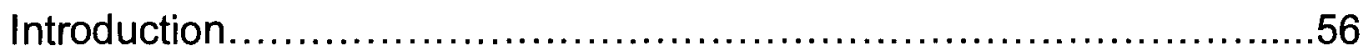




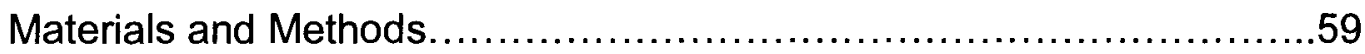

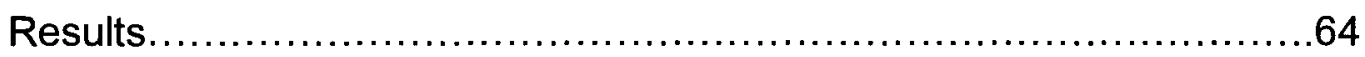

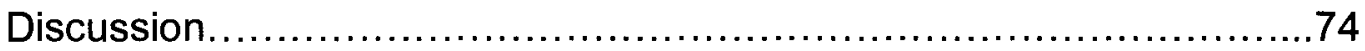

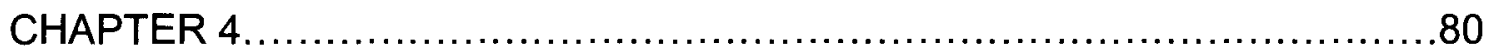

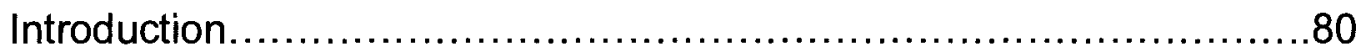

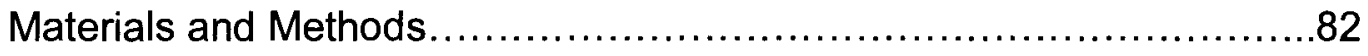

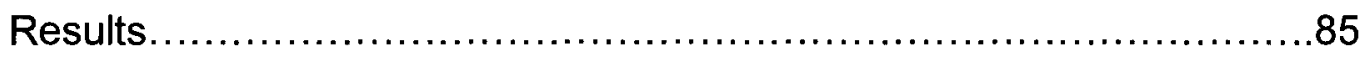

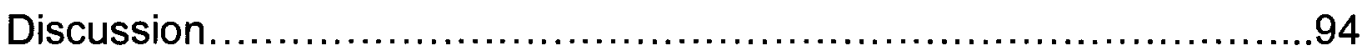

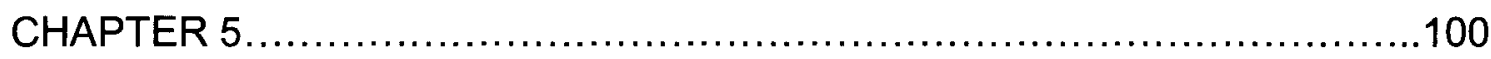

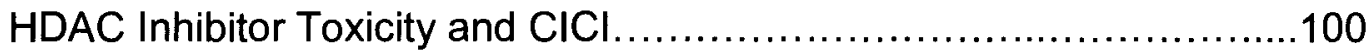

Dualism of HDAC Inhibition............................................109

Future HDAC Inhibitor Development and Therapy....................115

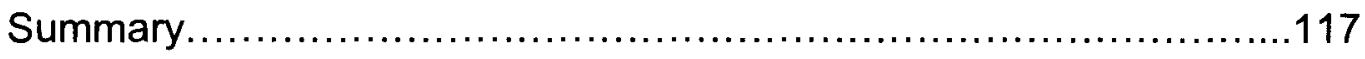

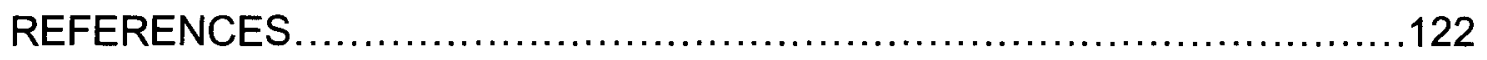

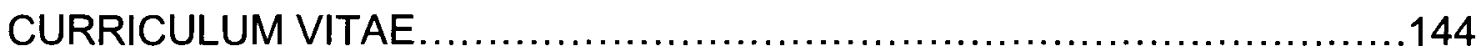




\section{ABSTRACT \\ HISTONE DEACETYLASE INHIBITION-MEDIATED \\ CYTOTOXICITY OF OLIGODENDROCYTES}

Toros A. Dincman

August 23, 2011

Recent studies indicate post-translational deacetylation by members of the superfamily of histone deacetylase complexes (HDACs) is necessary for oligodendrocyte precursor cell (OPC) differentiation into mature oligodendrocytes (OLs). However, it remains unknown if transient HDAC inhibition may promote OPC survival as has been shown in other cell types and neurological disease models. Chapter 2 demonstrates the development of a novel primary culture of OPCs from mouse pup cortices enriching the $\mathrm{O}^{+} \mathrm{OPC}$ population using Magnetic Activated Cell Sorting (MACS) technology. Once plated, OPCs were glial fibrillary acidic protein $\left(\mathrm{GFAP}^{-}\right), \mathrm{A}^{-} \mathrm{B5}^{+}, \mathrm{NG}^{+}$, and $\mathrm{O}^{+}$. When induced to differentiate, mouse OPCs became either more complex $\mathrm{O}^{+}$and $\mathrm{O} 1^{+}$ oligodendrocytes (OLs) or GFAP ${ }^{+}$astrocytes. Bi-potentiality is lost in co-culture, however, with rat embryonically derived dorsal root ganglia (DRG) as mature OLs aligned with DRG neurites.

In Chapter 3, following OPC treatment with the FDA approved pan-HDAC inhibitor suberoylanilide hydroxamic acid (SAHA), approximately $65 \%$ reduction in cell survival relative to vehicle-treated OPCs was observed. SAHA-mediated death of OPCs induces apoptosis partly by caspase activation with improvement 
of OPC survival when SAHA is accompanied by treatment with the general caspase inhibitor q-vd-oph. SAHA treatment of CNP-EGFP transgenic mouse pups increased the number of caspase activated apoptotic cortical OPCs compared to vehicle treated pups $(p<0.05)$.

In Chapter 4, we extended this study to spinal cord-derived rat primary OPCs to determine putative mechanism(s). Rat OPCs were similarly susceptible to SAHA-mediated HDACi. SAHA treatment of rat OPCs increased phosphorylated histone $\mathrm{H} 2 \mathrm{~A} . \mathrm{X}^{+}(\mathrm{YH} 2 \mathrm{AX})$ indicating the presence of HDACimediated DNA double-strand breaks. SAHA treatment also increased phosphorylated p53 and cleaved caspase 3 levels suggesting HDACi P53 mediated, caspase-dependant rat OPC apoptosis.

These results provide strong evidence of HDACi-mediated apoptosis of normal mouse pup and adult rat OPCs. SAHA is currently involved in more than 180 clinical trials, some of which include a pediatric patient population, and is in consideration for use in the treatment of psychiatric and neurodegenerative conditions. These results strongly suggest that such clinical use of SAHA may negatively impact OPC survival and potentially be detrimental to the myelinating brain and spinal cord. 


\section{LIST OF FIGURES}

FIGURE

PAGE

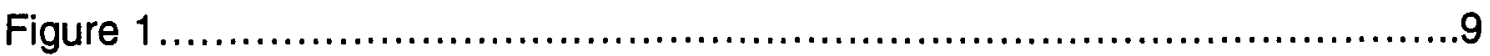

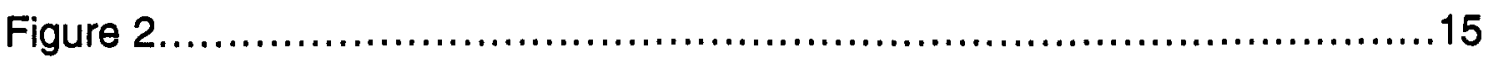

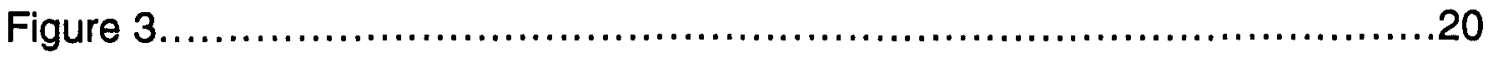

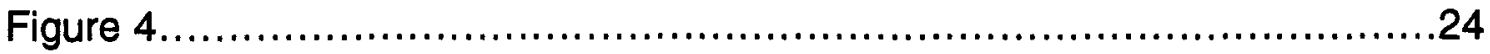

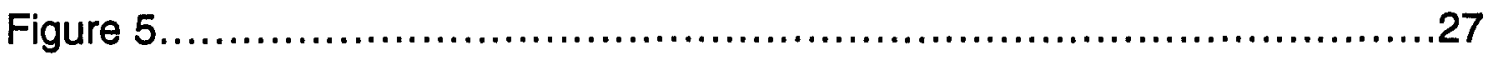

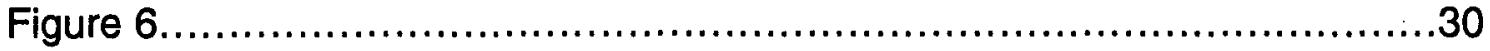

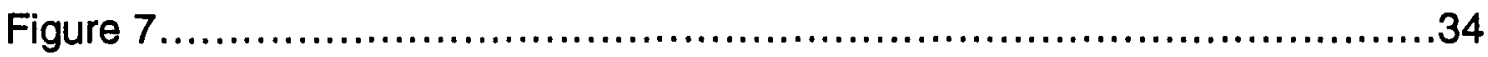

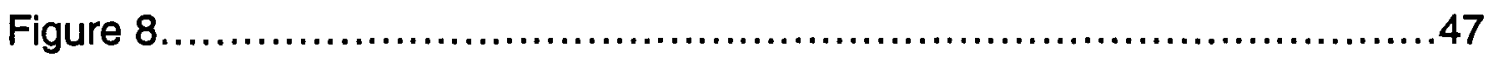

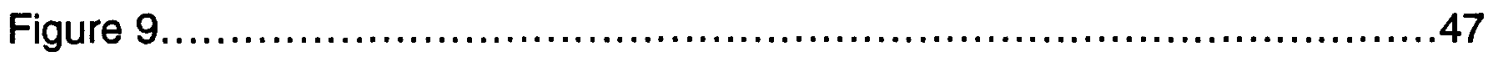

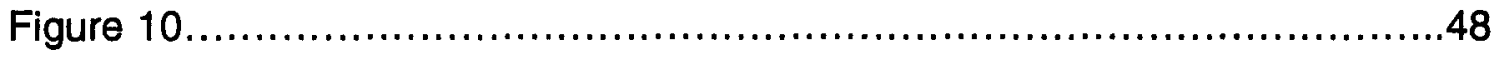

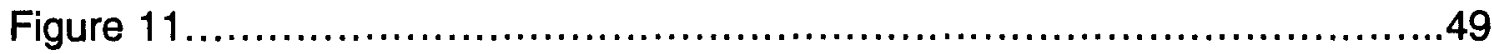

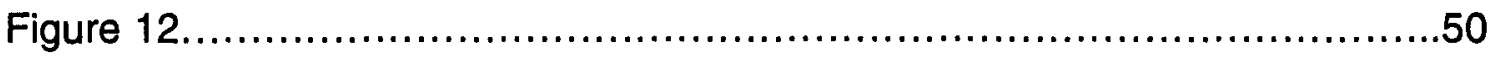

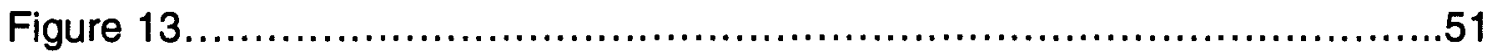

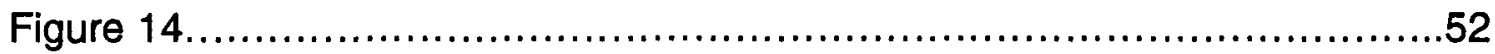

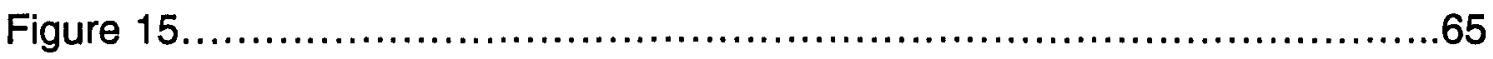

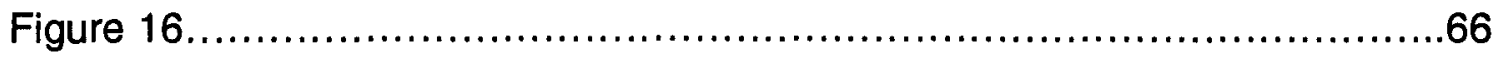

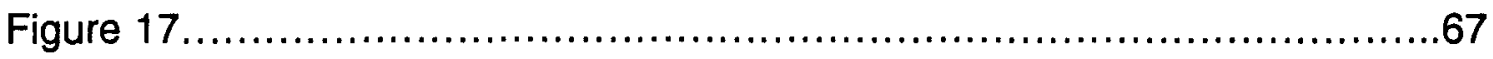

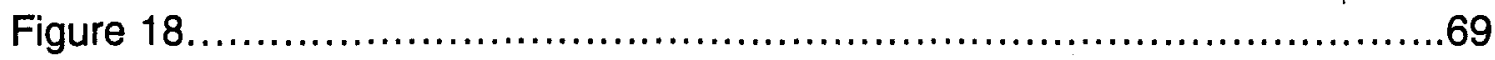

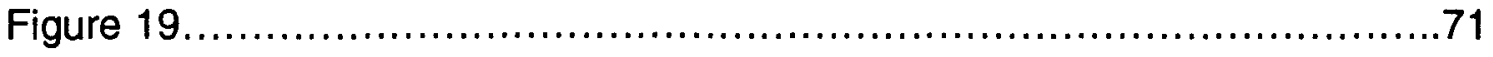




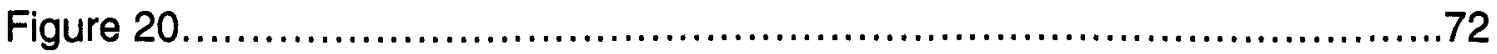

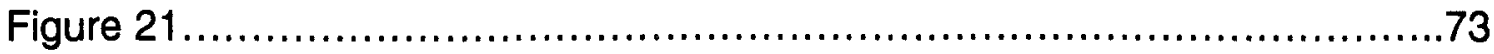

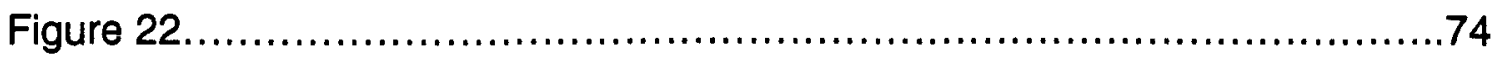

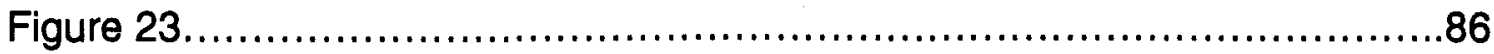

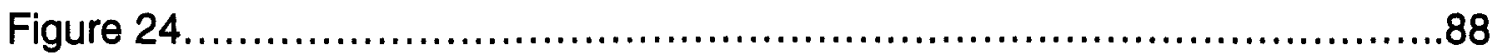

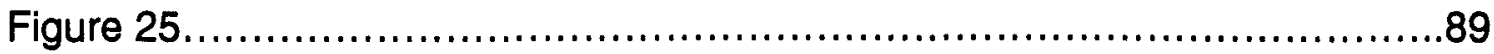

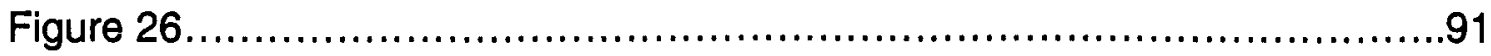

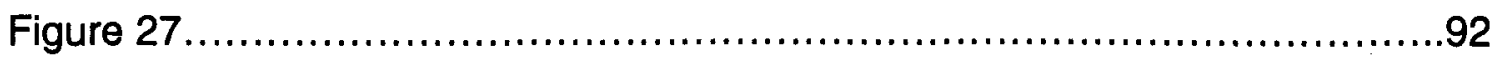

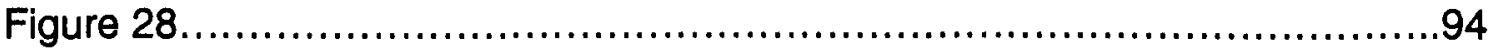

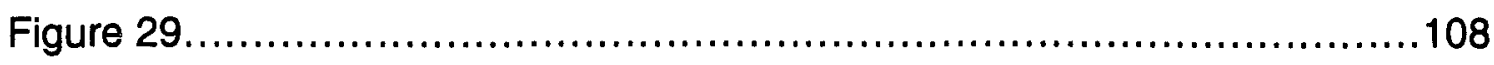

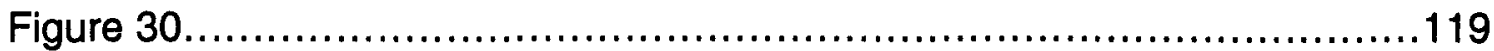




\section{CHAPTER 1}

\section{INTRODUCTION}

\section{SCI INCIDENCE AND PATHOLOGY}

$\mathrm{SCl}$ is the second leading cause of paralysis in the United States with over 1.2 million people currently suffering from symptomology secondary to longstanding $\mathrm{SCl}$. SCl occurs most often in males $(61 \%)$ with the average age of those who reported paralysis secondary to $\mathrm{SCl}$ at 48 years old having, on average, being 14 years removed from their injury (www.cdrf.org). Estimated lifetime costs for a young male with a high cervical $\mathrm{SCl}$ are over $\$ 3$ million with over $\$ 770,000$ in costs during the first year of the injury (www.spinalcord.uab.edu). With both a pressing need to improve the quality of life for these patients and to help reduce individual and national healthcare costs, continued $\mathrm{SCl}$ research into new promising therapeutic modalities is absolutely necessary.

Spinal cord contusion results in immediate cell death due to extensive hemorrhage and sheer force of the impact causing significant cellular membrane disruption. These primary processes result in widespread demyelination, oligodendrocyte (OL) and neuronal cell death, microvascular dysfunction, and a massive inflammatory response. Secondary processes characterized by 
continuing oligodendroglial apoptosis and subsequent demyelination, chronic presence of activated inflammatory cells, Wallerian degeneration, and ultimately the formation of a glial scar surrounding a cyst-like cavitation result in the gradual development of the full destructive pathology of the injured spinal cord (Hall and Springer, 2004; Totoiu and Keirstead, 2005; Thuret et al., 2006; Ankeny and Popovich, 2009).

$\mathrm{SCl}$ pathological hallmarks also consist of often spared tissue that may play an important role in functional recovery. Near the injury epicenter, a rim of intact, myelinated axonal tracts is often present (Sahni and Kessler, 2010). However, as this is observed in otherwise neurologically complete lesions following human $\mathrm{SCl}$, its relevance, particularly in spontaneous recovery following $\mathrm{SCl}$, remains unclear. Furthermore, endogenous reparative processes

are activated immediately following SCl (Thuret et al., 2006). These largely come in two forms to fulfill two different purposes: (1) to limit the extent of tissue damage and isolate the site of injury and (2) to replenish lost cellular populations. Though it is often debated how beneficial the former is, it is well accepted that latter may be futile unless therapeutically enhanced.

\section{OLIGODENDROCYTES: RELEVANCE AND DEVELOPMENT Oligodendrocytes in disease}

Of all endogenous responses that involve cell proliferation and migration into the $\mathrm{SCl}$ epicenter, oligodendrocyte precursor cells (OPCs) are among the most relevant and best described (Jones et al., 2002; Thuret et al., 2006; 
McTigue and Tripathi, 2008; Sahni and Kessler, 2010). However, there are number of CNS diseases whose pathogenesis includes OL death and subsequent failure of endogenous OPCs to lead a complete remyelinative response. Aside from $\mathrm{SCl}, \mathrm{OL}$ loss mediated by acute inflammation followed by atrophy of demyelinated axons is classically defined in the progression of multiple sclerosis (MS). As current immunomodulatory molecular therapies target early MS lesions, these do not provide improvement of chronic/progressive phase of disease nor directly facilitate OPC migration, renewal, and/or maturation at lesions despite limited remyelination mediated by an endogenous OPC and stem cell response (Fancy et al., 2010; Kremer et al., 2011).

Additionally, Periventricular leukomalacia (PVL), which can lead to cerebral palsy in affected premature infants, display abnormal myelination patterns relative to normal brains. However, this appears to be more specific to myelin formation rather than $\mathrm{OL}$ death as there was no significant difference in Olig2 cell density in PVL affected brains (Billiards et al., 2008; Fancy et al., 2010). Extensive reduction in myelination and OL death is also seen following ischemic brain injury and stroke (Zhang and Chopp, 2009). White matter damage is also evident in Alzheimer's disease (AD) pathogenesis. Amyloid- $\beta$ (an extracellular peptide that serves as a pathological hallmark of $A D$ ) mediated toxicity of OLs is present in AD brains (Xu et al., 2001; Roth et al., 2005). Furthermore, increasing evidence implicates primary oligodendrogliopathy with histopathological features showing OL loss, dysmyelination and subsequent axonal loss in another neurodegenerative disease, multiple system atrophy 
(MSA), (Wenning et al., 2008). The neurodegenerative genetic disorder Huntington's disease (HD) also displays pathological hallmarks of myelin disruption with loss of cerebral white matter accompanying cortical gray matter loss (Fennema-Notestine et al., 2004).

Psychiatric disorders such as schizophrenia have considerable OL involvement. Increasing evidence suggests OL dysfunction and myelin disruption may in fact be the primary alterations in schizophrenia (Takahashi et al., 2011). Additionally, mood disorders such as bipolar disorder demonstrate considerable evidence of white matter deficits. These seem to be linked to dysregulation of myelin-associated gene expression, particularly neuregulin-1 (NRG1) of which genetic polymorphisms have been identified to be associated with the disease (McIntosh et al., 2009). Overall, there is a large variety of CNS diseases that involve $\mathrm{OL}$ death and/or dysfunction either as a primary or secondary cause of disease typically resulting in axonal myelination abnormalities ultimately followed by neurological deficits.

\section{Oligodendrocytes: Maturation markers}

OPCs are present in both the developing and the adult mammal (Wolswijk and Noble, 1989; Noble et al., 1992; Nishiyama, 1998; Emery, 2010; Trotter et al., 2010). Although the adult CNS maintains a proliferative population of adults OPCs that respond to CNS injury and disease, it's reparative/replacement response is often insufficient to achieve full remyelination and complete functional recovery, as is the case following SCl (Cao et al., 2005; Totoiu and Keirstead, 2005; McTigue and Tripathi, 2008). Therefore, understanding OPC 
maturation under developmental conditions to improve upon endogenous responses will facilitate improvement of adult OPC proliferation and maturation following $\mathrm{SCl}$ and other CNS injuries and diseases.

OPC maturation into myelinating OLs is a highly dynamic process with each step involving reliable expression of cell surface markers indicative of the maturation state (Fig. 1). The sulfatide A2B5 and the chondroitin sulfate proteoglycan NG2 (nerve/glial antigen 2) are found to be co-expressed early in OPC development and are useful markers indicative of bi-potential OPCs, although much debate surrounds $\mathrm{NG}_{2}{ }^{+}$cells and there lineage commitment or its putative classification as fourth major glial cell type (Nicolay et al., 2007; McTigue and Tripathi, 2008; Nishiyama et al., 2009; Trotter et al., 2010; Mangin and Gallo, 2011). This is accompanied by platelet-derived growth factor a-receptor (PDGFaR) expression as well as early expression of oligodendroglial lineage specific transcription factors Olig1 and Olig2 (Nicolay et al., 2007; Nishiyama et al., 2009).

As OPC maturation continues, other cell surface sulfatides such as $\mathrm{O} 4$ and $\mathrm{O} 1$ are concomitantly synthesized with increased process formation of OPCs. It is important to note that $\mathrm{O} 4$ serves as a reliable marker for the late proliferative OPC whereas 01 is indicative of the non-proliferative, premyelinating, maturing/mature OL (Nicolay et al., 2007; Nishiyama et al., 2009; Cao et al., 2010). Finally, the most reliable indicator for the formation of a mature, myelinating $\mathrm{OL}$ is the synthesis of myelin basic protein (MBP) often observed in 
membranous sheaths outstretched from a highly arborized OL (Nicolay et al., 2007; Trotter et al., 2010).

\section{Oligodendrocyte maturation: Permissive factors}

In vivo studies of vertebrate models and in vitro investigations using either oligodendroglial cell lines and/or primary OPC cultures have provided great insight into the tightly regulated and complex interplay of transcription factors and extracellular signals involved in OPC differentiation into a mature, myelinating $\mathrm{OL}$ (Fig. 1). Early in OPC maturation, expression of basic helix-loop-helix (bHLH) transcription factors OLIG1 and OLIG2 is observed and maintained throughout OPC differentiation into OLs (Nicolay et al., 2007). Their function is necessary for proper oligodendroglial lineage specification and OL differentiation (Zhou and Anderson, 2002; Ligon et al., 2006; Nishiyama et al., 2009). Acting synergistically with Olig2, a member of the SRY-related HMG-box family of transcription factors, Sox10 has been demonstrated to play a pivotal role in OL maturation (Nicolay et al., 2007). In vitro siRNA knockdown of Sox10 resulted in decreased differentiation of OPCs into $\mathrm{MBP}^{+}$OLs (Dugas et al., 2006). Furthermore, overexpression of Sox10 in embryonic chick spinal cords caused an increase in expression of mature $\mathrm{OL}$ markers such as MBP and proteolipid protein (PLP) as well as Olig2 and Nkx2.2, transcription factors permissive to OL maturation (Liu et al., 2007b).

Though their mechanisms of action remain uncertain, the homeobox proteins Nkx2.2 and Nkx6.2 also play a pivotal role in the formation of mature, myelinating OLs (Talbott et al., 2005; Nicolay et al., 2007; Cai et al., 2010). 
Nkx2.2 knockout mice results in decreased MBP expression in the embryonic medulla and reduced PLP expression in the P7 forebrain (Qi et al., 2001). Additionally, Ngn3 knockout mice, which have reduced Nkx2.2 expression in the embryonic spinal cord, also have decreased expression of mature OL markers PLP and MBP postnatally (Lee et al., 2003). Nkx6.2 expression is concomitant with expression of mature OL markers PLP and MBP (Awatramani et al., 1997). Furthermore, Nkx6.2 is required for normal myelin formation as determined by ultrastructural analysis in Nkx6.2 null mice (Southwood et al., 2004). Nkx6.2 and $\mathrm{Nkx} 2.2$ expression is tightly coordinated in Olig2 ${ }^{+}$OLs as evidenced by early Nkx2.2 expression in OPCs followed by its down-regulation and increased Nkx6.2 expression in mature OLs in the mouse postnatal spinal cord (Cai et al., 2010). Lastly, the extracellular ligand neuronal growth factor Neuregulin-1 (NRG1) has also been demonstrated to promote OL differentiation in vitro and results in hypermyelination following CNS NRG1 overexpression (Calaora et al., 2001; Brinkmann et al., 2008). However, NRG1 appears to be not necessary for CNS myelination as other promyelinating factors are likely sufficient (Emery, 2010).

Another important mediator of OPC differentiation into mature OLs is the thyroid hormone $(\mathrm{TH})$ triiodothyronine $\left(\mathrm{T}_{3}\right)$ which increases OL-mediated myelination of neurites in spinal cord explants (Park et al., 2001). Reduced MBP and PLP expression is seen in the corpus callosum of hypothyroid animals (Ferreira et al., 2007). Additionally, $\mathrm{T}_{3}$ treatment of rats that underwent curpizoneinduced demyelination of the corpus callosum showed improved histological 
outcome relative to saline-treated animals (Franco et al., 2008). Another investigation showed restoration of corpus callosum myelination by diffusion tensor magnetic resonance imaging (DT-MRI) in $\mathrm{T}_{3}$ infused mice following cuprizone-induced demyelination relative to controls (Harsan et al., 2008). This promotion of OPC maturation by $\mathrm{TH}$ appears to be mediated by $\mathrm{TH}$ receptor alpha 1 (TRa1) as TH- treated optic nerve cultures results in decreased percentage of $\mathrm{O}^{+} \mathrm{OLs}$ in the absence of TRa1 (Billon et al., 2002).

Though mechanisms are still undefined, neuronal activity also appears to play a role in OL differentiation (Emery, 2010). Whether by modulation of expression of axonal surface ligands or increasing promyelinating factor release by astrocytes, both direct and indirect mechanisms have been postulated connecting neuronal electrical activity to the promotion of OL maturation (Itoh et al., 1995; Ishibashi et al., 2006). Additionally, direct stimulation by the neurotransmitter glutamate to glutamate responsive OPCs result in small excitatory postsynaptic potentials that regulate OPC behavior as well (Barres et al., 1990; Ziskin et al., 2007; Karadottir et al., 2008; Emery, 2010). However, OPC neurotransmitter release onto OPC may also be important in maintaining the OPC pool as in vitro treatment of OPCs with glutamate resulted in a block in rectifier potassium channels and inhibited subsequent $\mathrm{OL}$ differentiation (Gallo et al., 1996; Emery, 2010). Though it remains unclear what role neuronal activity may have on differing OPCs populations, neuronal electrical activity nonetheless plays a significant role in regulating OPC development and maturation. 


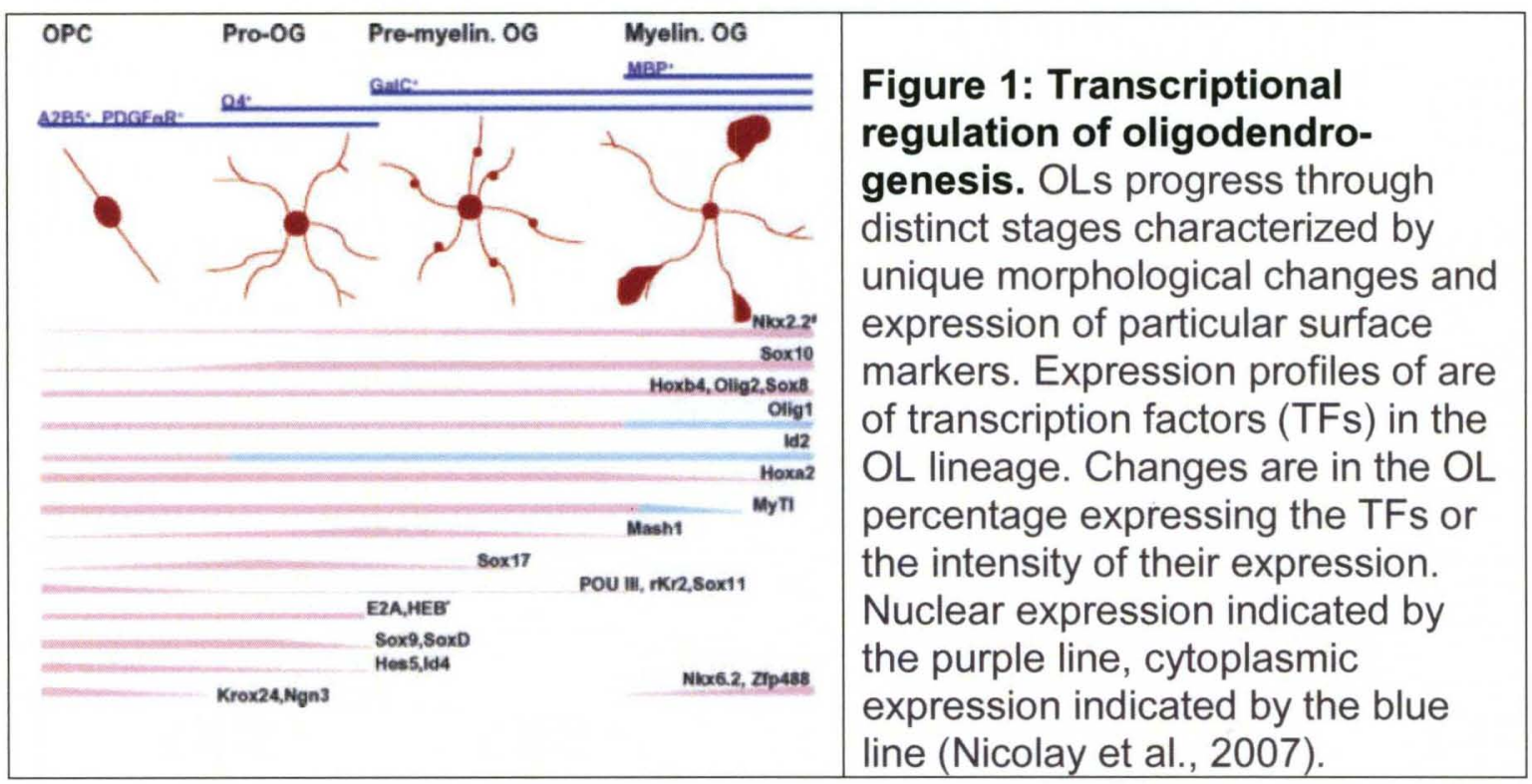

\section{Oligodendrocyte maturation: Inhibitory factors}

In addition to molecular mediators permissive to $\mathrm{OL}$ maturation, there are also a number of developmentally identified factors that inhibit OPC differentiation into mature, myelinating OLs (Fig. 1). Bone morphogenetic protein (BMP) signaling has been well characterized in its role in inhibiting OPC maturation and oligodendroglial lineage commitment (Nicolay et al., 2007). Treatment of E16 mixed rat cortical cultures with BMP2 reduced the number of $\mathrm{O}^{+}$OPCs. Treatment with the BMP receptor antagonist Noggin, resulted in the opposite effect (Mabie et al., 1999; Nicolay et al., 2007). Transgenic mice overexpressing BMP4 lead to increased astrocyte lineage commitment rather than OL across the mouse brain (Gomes et al., 2003). Recently, reactive astrocytes from the injured rat spinal cord have been shown to have increased BMP expression and this expression counteracted OPC maturation to OLs. 
Following Noggin treatment, the effect was reversed resulting in increased OPC maturation into OLs and reduced astrocyte formation (Wang et al., 2011).

Furthermore, active BMP signaling leads to increased expression of helixloop-helix $(\mathrm{HLH})$ transcription factors Id 2 and Id 4 that negatively regulate OPC differentiation into mature OLs (Samanta and Kessler, 2004; Nicolay et al., 2007). In cultured OPCs, Id2 and Id4 expression results in reduced OPC maturation into GalC ${ }^{+}$OLs (Kondo and Raff, 2000; Wang et al., 2001). As reduced levels of the pro-OL transcription factors Olig1 and Olig2 are concomitant with increased Id 2 and Id4, they may play a role in directly reducing Olig1 and Olig2 expression thereby reducing OL maturation (Fu et al., 2002). Furthermore, Id 2 and Id4 appear to exert their inhibitory effect on OL formation in $\mathrm{NG}^{+} / \mathrm{O} 4^{-} \mathrm{OPCs}$, which is relatively early in the oligodendroglial lineage (Gokhan et al., 2005). Therefore, whether direct or indirect via induced inhibitory transcription factor expression, the BMP signaling pathway is a critical component in preventing OPC maturation into fully mature OLs.

In addition to BMP involvement, the single-pass transmembrane protein Notch has also been implicated in preventing OL formation (Nicolay et al., 2007; Jessen and Mirsky, 2008). Notch pathway activation with its ligand Jagged prevented reduced in vitro differentiation into $\mathrm{GaIC}^{+} \mathrm{OLs}$ in rat optic nerve isolated OPC cultures (Wang et al., 1998). In the developing mouse, Cremediated removal of the Notch1 gene resulted in increased number of OPCs and their early appearance in the spinal cord. However, the effect is so profound that OL differentiation was premature and Notch1 ablated mice had increased OL 
apoptosis at birth as well as a loss of spatio-temporal regulation of $\mathrm{OL}$ differentiation throughout the CNS (Genoud et al., 2002). Additionally, Notch1 null heterozygous mice displayed increased MBP and PLP expression throughout the brain up to 2 weeks postnatally (Givogri et al., 2002).

Downstream expression of the transcription factor Hes5 following Notch activation is also inhibitory towards OPC maturation (Nicolay et al., 2007; Li and Richardson, 2009; Emery, 2010). Putative Hes5 interaction with pro-OL maturation transcription factor Sox10 indirectly reduces MBP expression and subsequent OPC maturation in an oligodendroglial cell line (Liu et al., 2006). However, the involvement of the Hes family of transcription factors may be more complex. Though Hes5 reduces MBP expression, it also reduces overall glialrestricted precursor (GRP) cell differentiation regardless of cell fate, whereas Hes1 preferentially promotes astrocyte differentiation over OLs (Wu et al., 2003). Overall, activation of the Notch signaling pathway likely via reduced expression of Hes family members reduces OPC maturation. Nonetheless, as Notch remains important in preventing premature OL formation, further understanding of the timing of its inhibitory effects is not clearly defined.

\section{SIGNIFICANCE OF HDAC ACTIVTY}

\section{Histone acetylation and deacetylation}

An important DNA transcriptional regulatory mechanism in eukaryotic cells is modulation of chromatin structure. One form of this consists of reversible posttranslational covalent modification of "histone tails", largely on histone $3(\mathrm{H} 3)$ and histone 4 (H4) nucleosome subunits (Grunstein, 1997; Cheung et al., 2000; 
Haberland et al., 2009). The resulting alteration of nucleosomal structure upstream, downstream or within a specific gene(s), subsequently changes transcriptional expression patterns of that gene(s). Of known covalent modifications, acetylation and deacetylation of $\varepsilon$ lysine amino groups in histone tail regions of $\mathrm{H} 3$ and $\mathrm{H} 4$ subunits tightly regulates transcription factor accessibility to DNA binding sites that regulate expression of target gene(s) in a modulated region (Shahbazian and Grunstein, 2007). Certain DNA binding sites become accessible as the addition of an acetyl group to these lysine residues results in the neutralization of their positive charge thereby preventing interaction between the histone tail and the negatively charged DNA backbone. In contrast, deacetylation results in DNA inaccessibility and loss of gene expression (Haberland et al., 2009).

As histone acetylation depends on the activity of both histone acetyltransferases (HATs) and histone deacetylase complexes (HDACs), it is the superfamily of HDACs that has received considerable attention due to the number of isoforms, the redundancy of their function, and the specificity of their targets. Classically, 11 HDAC isoforms have been characterized (excluding the 7 $\mathrm{NAD}^{+}$dependent class III HDACs called Sirtuins). Classes I (HDAC1, 2, 3, and 8), Ila (HDAC4, 5, 7, and 9), Ilb (HDAC6, and 10), and IV (HDAC11) HDACs are zinc-dependent metalloproteins largely involved in deacetylation of acetyl groups located on lysine residues of histone tails (Fig. 2). HDAC1 and HDAC2 have relatively redundant roles as illustrated in their association with transcriptionally repressive multi-subunit complexes (Yang and Seto, 2003). However, HDAC 
gene and cellular specificity is demonstrated by HDAC3 in its association with an entirely different transcriptional complex compared to HDAC1 and HDAC2, by HDAC4 and HDAC9 in their tissue expression specificity in the brain, and by HDAC6 in its role as the primary mammalian cytoplasmic deacetylase (Zhang et al., 2002; Yang and Seto, 2003; Vega et al., 2004; Zhang et al., 2008).

\section{HDAC inhibitors and their biological effects}

HDAC inhibitors are a varied class of small molecule compounds with a high therapy potential that were initially developed for use in anti-cancer therapies, though their spectrum of therapeutic potential beyond anti-cancer treatment is broadening quickly (Fig. 2) (Abel and Zukin, 2008; Hahnen et al., 2008; Bertrand, 2010; Colussi et al., 2010; Marks, 2010). As mentioned above, this modification typically results in heterochromatin modification and transcriptional silencing, however, it may also result in increased transcription of other genes such as the cell cycle protein p21/WAF1/CIP1 (Richon et al., 2000; Sandor et al., 2000; Lane and Chabner, 2009; Marks, 2010). As a whole, inhibiting HDAC activity results in increased levels of acetylated histone and global transcriptional changes that favor expression of genes promoting apoptosis and cell cycle arrest (Fig. 3) (Bertrand, 2010; Marks, 2010). Additionally, they also play a significant role in post-translational deacetylation of other proteins both nuclear and cytosolic as evidenced by stabilization of p53 and a-tubulin following HDAC inhibition (HDACi) (Gu and Roeder, 1997; Zhang et al., 2003; Lane and Chabner, 2009). Lastly, HDACs have also been shown to be important in the regulation of the extrinsic apoptotic pathway as HDACi increased 
expression of Fas, TNF- $\alpha$, and TRAIL death receptor proteins (Insinga et al., 2005; Nebbioso et al., 2005; Lane and Chabner, 2009).

Irregular recruitment HDACs to aberrant fusion proteins were identified in acute myelocytic leukemia (AML) and acute lymphoblastic leukemia (ALL) (Bolden et al., 2006; Marks, 2010). Increased expression of largely class I HDAC isoforms relative to normal tissues have been reported in many human cancers that include gastrointestinal, prostate, non-small cell lung, and breast cancers (Bolden et al., 2006; Marks, 2010). Furthermore, siRNA mediated knockdown of individual HDAC isoforms resulted in decreased tumor cell growth and survival in a number of human tumor cell lines (Bolden et al., 2006). These observations continued the further development of HDAC inhibitors and spawned the extensive work for their use as a treatment against a range of malignancies. Primarily five classes of HDACi's have been extensively studied thus far and are delineated based on their chemical structure. The short-chain fatty acids (aliphatic acids) that include phenylbutyrate and valproic acid (VPA), hydroxamates which include trichostatin A (TSA) and the FDA approved suberoylanilide hydroxamic acid (SAHA), benzamides such as MS-275, cyclic peptides such as depsipeptide (romidepsin), and eletrophilic ketone derivatives (Bolden et al., 2006; Bertrand, 2010; Marks, 2010). Within and between classes, there is variability on HDAC isoform inhibitory specificity. VPA is largely a class I HDAC inhibitor, TSA and SAHA are pan-HDAC inhibitors, whereas MS-275 is inhibitory towards HDAC1 at low concentrations and the remaining class I HDACs at higher concentrations (Bolden et al., 2006; Bertrand, 2010). 


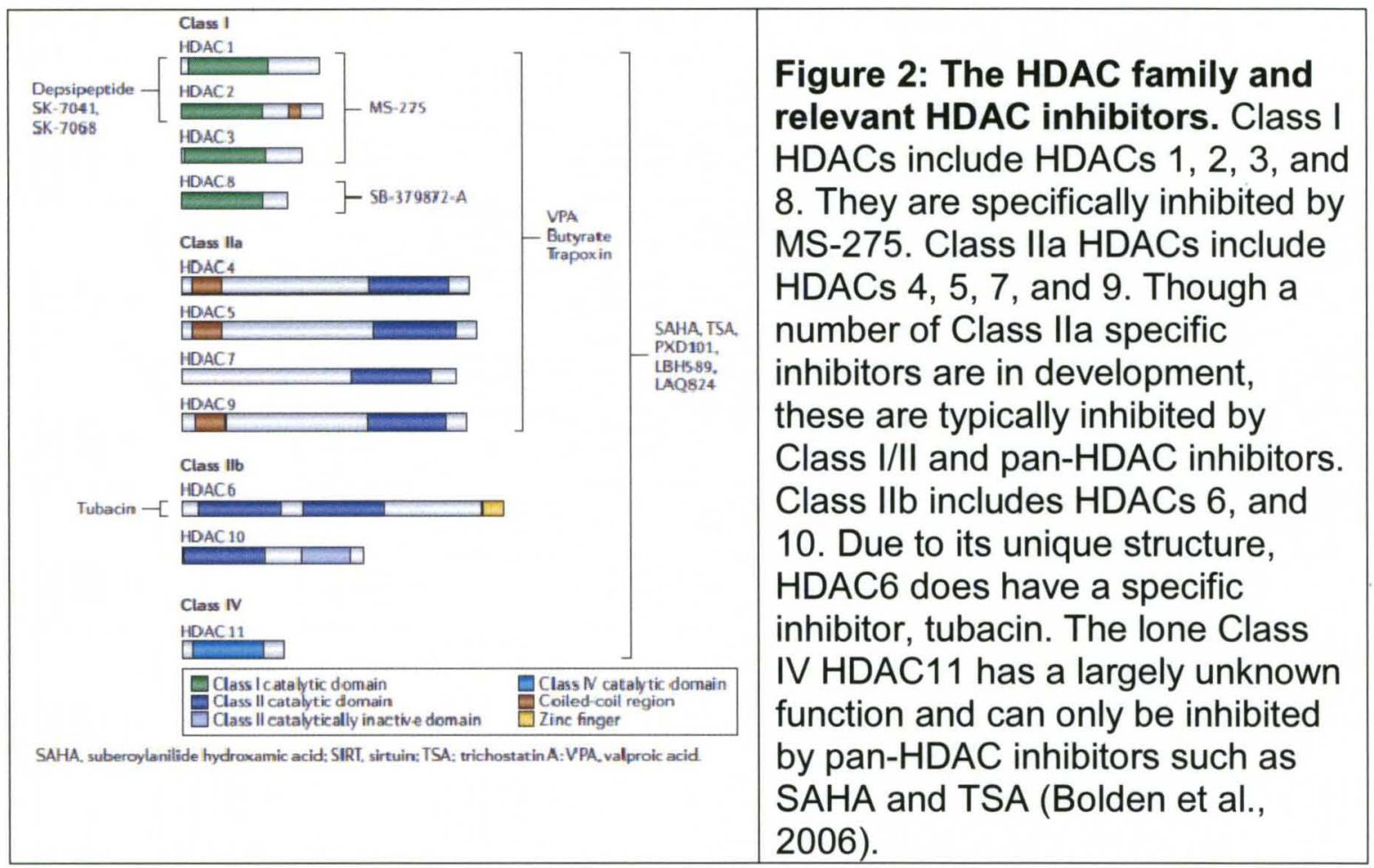

\section{Role of HDACs in OPC maturation}

HDAC mediated chromatin remodeling resulting in transcriptional changes is a key regulator of OPC maturation into OLs (Yu et al., 2010). In vitro and in vivo studies indicate that HDAC-mediated histone deacetylation is necessary for OL differentiation (Marin-Husstege et al., 2002; Shen and Casaccia-Bonnefil, 2008). In fact, HDACi-induced OPC developmental plasticity restricts them from a mature $\mathrm{OL}$ fate and increases their differential potential to include a neuronal lineage (Lyssiotis et al., 2007). In rat brains, the status of HDAC activity determines OPC differentiation into a glial or neuronal fate (Liu et al., 2007a). Furthermore, VPA-mediated HDACi in neonatal rats resulted in hypomyelination of the corpus callosum due to reduction of OPC differentiation into mOLs (Shen et al., 2005). In the rat OL-1 oligodendroglia cell line, HDACi by TSA resulted in 
reduced gene expression of myelin basic protein (MBP) and proteolipid protein (PLP), both of which are expressed in mOLs. Moreover, siRNA inhibition of HDAC11 mediated deacetylation of $\mathrm{H} 3$ and $\mathrm{H} 4$ corresponding to the MBP and PLP genes prevented OPC differentiation to mature OLs (Liu et al., 2009).

Recently, it was demonstrated that VPA-mediated HDACi in cuprizonetreated adult mice prevented remyelination of the corpus callosum due to reduced OPC differentiation (Shen et al., 2008). Furthermore, HDAC1/2mediated disruption of the Wnt signaling pathway are necessary for $\mathrm{OL}$ maturation (Li and Richardson, 2009; Ye et al., 2009). These data implicate HDACs in a global repressive role in OPC differentiation into mature OLs (Shen and Casaccia-Bonnefil, 2008) and suggest that increasing rather than inhibiting HDAC activity in OPCs will lead to increased maturation into mOLs and be therapeutically applicable in demyelinating disease(s). Recently, increased histone acetylation was observed in frontal lobe white matter of chronic MS patients relative to non-neurological controls with concomitant increase in expression of OL maturation inhibitors suggesting reduced HDAC activity efficiency as during MS progression (Pedre et al., 2011). However, it was also recently demonstrated that suberoylanilide hydroxamic acid (SAHA) mediated pan-HDACi preserved white matter in ex vivo explants of optic nerve under oxygen and glucose deprivation suggesting a putative role HDACi promotion of OL survival under ischemic conditions (Baltan et al., 2011). Overall, these studies suggest HDAC activity plays an important role in promoting OPC 
maturation, however, may have a putative role in improving OL survival under certain conditions.

\section{Role of HDACs in CNS Disease}

Despite concerns over the role of HDACi on OPC maturation, altered HDAC activity has been implicated in a number of neurodevelopmental and neurodegenerative diseases where HDACi show to be beneficial (Kazantsev and Thompson, 2008). Among these, the role of HDACs in the polyglutamine-repeat disorders such as Huntington's disease (HD) has been most extensively studied. Though a number of cellular perturbations occur in HD, one of the most relevant are changes in global histone acetylation patterns and chromatin structure (Butler and Bates, 2006). HDAC3 activity increases polyglutamine-mediated toxicity in a C. elegans $\mathrm{HD}$ model via dysregulation of gene expression when associated with co-repressors. HDAC3 targeted siRNA knockdown reduced such toxicity in the same animal model (Fischle et al., 2002; Bates et al., 2006). Additionally, HDAC5 nuclear localization is elevated in HD brains, leading to speculation of a role in association with HDAC3 (Hoshino et al., 2003; Butler and Bates, 2006).

Experimental models of neurodevelopmental diseases such as Fragile $X$ syndrome, Friedrich's ataxia, and SMA have also shown improvement with HDAC inhibitor treatment (Kazantsev and Thompson, 2008). In vitro models of Fragile $X$ syndrome had transcriptional reactivation of the FMR1 gene when treated with HDAC inhibitors, a gene silenced in this disease (Chiurazzi et al., 1999). HDAC inhibitor treatment of cell-based models for Friedrich's ataxia relieved hypoacetylated mediated gene silencing of the frataxin gene that is 
typically observed in this disease (Herman et al., 2006; Kazantsev and Thompson, 2008). Increased HDAC2 levels are found just upstream of the transcriptional start site of the survival motor neuron2 (SMN2) gene- a protein insufficiently expressed in SMA (Hirtz et al., 2005; Kernochan et al., 2005; Avila et al., 2007). HDACi in brain slices from SMA patients increased SMN2 expression, resulting in a new putative therapy for SMA currently in clinical trials (Hahnen et al., 2006).

Lastly, HDACs have been implicated in the development of depression and schizophrenia (Tsankova et al., 2007; Abel and Zukin, 2008). In preliminary animal studies, deacetylation mediated by HDAC5 may play a role in antidepressant activity (Tsankova et al., 2006). Likewise, sodium butyrate-mediated HDACi in mice resulted in anti- depressant like activity (Schroeder et al., 2007). Most importantly, recovery of learning and memory deficits via dendritic sprouting and increased neuronal synapses following HDAC inhibitor treatment were seen in mice that displayed a number of cognitive deficits (Fischer et al., 2007). Overall, these data strongly suggest that HDACi may play a role in disease process attenuation for a number of neurodevelopmental and psychiatric disorders. However, it is important to keep in mind that the development of pharmacological HDAC inhibitors was geared towards use as a chemotherapeutic agent in conjunction with DNA damaging agents. Therefore, in light of the toxicity they display towards transformed cells, an as of yet undefined toxicity towards normal cells of the CNS may be present. 
ROLE OF HDACS IN DNA REPAIR

HDACs in DNA damage and repair

Whether originating from exogenous sources such as environmental genotoxic agents, ionizing radiation, or chemotherapeutic compounds or from endogenous sources ranging from regular DNA replication and repair events, replication fork collapse, or cellular metabolic intermediates, DNA is repeatedly bombarded by chemicals that can damage it (Hill and Lee, 2010). Therefore, repair of DNA damage is one of the most critical physiological functions any cell can undertake. This damage may occur in the form of DNA pairing mismatches, bulky adducts, base loss, or the formation of single-strand breaks (SSBs) and double-strand breaks (DSBs) (Jackson and Bartek, 2009). Depending on the type of damage, a wide variety evolutionarily conserved DNA damage response (DDR) mechanisms such as nonhomologous end-joining (NHEJ), homologous recombination (HR), nucleotide excision repair (NER), and base excision repair (BER) exist to counteract potential DNA damage (Lombard et al., 2005; Jackson and Bartek, 2009). However, if these mechanisms fail resulting in improper or inefficient DNA repair, then a mutation(s) may occur which could lead to cellular transformation or overwhelming accumulating DNA damage will result promoting cell death (Lombard et al., 2005; Jackson and Bartek, 2009). 


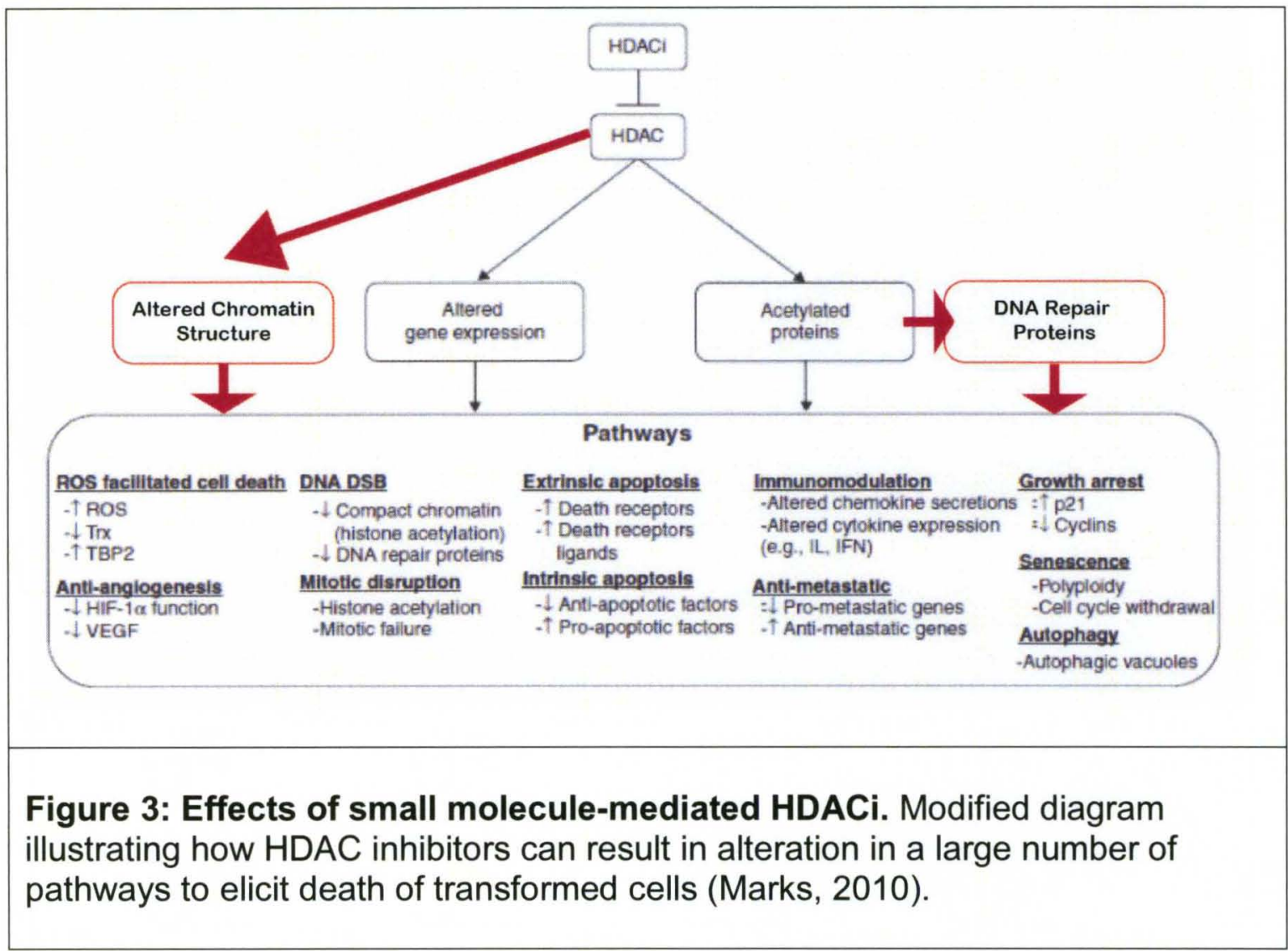

Of all of forms of DNA damage, DSBs are regarded as highly toxic and potentially most lethal, yet difficult for cells to repair (Abraham and Tibbetts, 2005; Jackson and Bartek, 2009; Hill and Lee, 2010). Failure to repair DSBs results in genomic instability such as chromosomal rearrangements or loss that can lead either to cancer or cell death (Jackson and Bartek, 2009; Hill and Lee, 2010). Cells primarily use either NHEJ of HR mechanisms to repair DSBs (Lieber, 2008; San Filippo et al., 2008; Jackson and Bartek, 2009). DDR in NHEJ repair require one, two, or more nuclear serine/threonine protein kinases: DNAdependant protein kinase (DNA-PK), Ataxia-telangiectasia mutated (ATM), and ATM and Rad3 related (ATR) (Abraham and Tibbetts, 2005; Cimprich and Cortez, 2008; Jackson and Bartek, 2009; Hill and Lee, 2010). Following DSB 
induction, DNA-PK catalytic subunit (DNA-PK $\mathrm{cs}_{\mathrm{cs}}$ ) is recruited to the site of damage and binds the Ku70/Ku80-damaged DNA complex after DSB recognition by Ku70/Ku80 (Smith and Jackson, 1999; Hill and Lee, 2010). DSB induction also results in Mre11, RAD50, and NBS1 (MRN Complex), which recognizes the site of damage and promotes activation of ATM via auto-phosphorylation (Abraham and Tibbetts, 2005). Ultimately, DSB activation of DNA-PK $\mathrm{C}_{\mathrm{cs}}$, ATM, and ATR promotes chromatin modification via phosphorylation of serine 139 of histone $\mathrm{H} 2 \mathrm{~A}$ variant $\mathrm{H} 2 \mathrm{AX}(\mathrm{\gamma H} 2 \mathrm{AX})$ on chromatin flanking DSB sites. This results in recruitment of other DDR proteins that both promote and amplify DSB repair and signaling (Jackson and Bartek, 2009). Ultimately, DNA-PK $\mathrm{cs}_{\mathrm{cs}}$ ATM, and ATR phosphorylate downstream effectors such as $\mathrm{p} 53$, which then subsequently promote cell cycle arrest and/or apoptosis (Abraham and Tibbetts, 2005; Lombard et al., 2005; Cimprich and Cortez, 2008; Jackson and Bartek, 2009; Hill and Lee, 2010).

Post-translational modification such as acetylation, phosphorylation, methylation, and ubiquitylation, which alter chromatin structure, play an important role in DNA repair (Fig. 4) (Misteli and Soutoglou, 2009). More specifically, acetylation status in proteins involved in DSB repair and histones as regulated by HATs and HDACs are relevant for efficient DSB repair (Misteli and Soutoglou, 2009; Miller et al., 2010). HDAC4 in human cell lines was previously shown to colocalize with p53 binding protein 1 (53bp1) following DSB induction by irradiation or etoposide with subsequent knockdown resulting in reduced DNA repair (Kao et al., 2003). Additionally, HDAC mediated deacetylation of Ku70, a component 
of DNA-PK, is necessary for its DNA binding capability and promotion of DSB repair following exogenous DSB induction in prostate cancer cells (Chen et al., 2007a). Considerable evidence also demonstrates sensitivity of transformed cell lines to combinatorial treatments consisting of both HDAC inhibitors and DNA (DSB) damage inducing agents (Karagiannis et al., 2007; Harikrishnan et al., 2008).

Acetylation of Lys9 and Lys56 of histone $\mathrm{H} 3$ (H3K9Ac and H3K56Ac) are DNA damage responsive modifications in human cells (Tjeertes et al., 2009). The role of HDAC activity in DSB repair was further explained by demonstrating HDAC1 and HDAC2 mediated hypoacetylation of H3K56 in response to DSB induction by phleomycin treatment of human cells. Subsequent HDAC1 and HDAC2 depletion maintained DNA damage signaling, which may be reflective of inefficient DSB repair (Miller et al., 2010). Furthermore, in Saccharomyces cerevisiae, HDAC inhibition/ablation counteracts activation of Mec1 (orthologue of human ATR) resulting in reduced DSB repair. This is mediated by acetylation of the recombination protein Sae2 (orthologue of Human CtBP interacting protein, CtIP), following $\mathrm{HDACi}$, which results in Sae2 degradation by promoting autophagy (Robert et al., 2011). However, much remains to be defined, particularly in normal CNS cells, in terms of the role HDACs have in maintaining genomic stability during typical cell processes and in the presence of endogenous and exogenous DNA damaging agents. 


\section{DNA damage and repair in OLs}

A majority of the studies examining DNA damage in OPCs and OLs demonstrate a seemingly enhanced susceptibility to oxidative stress than other cells (McTigue and Tripathi, 2008). This is largely attributed to its synthesis of large, membranous sheets of myelin which presents the highest metabolic demands on any cell of the CNS (Connor and Menzies, 1996; McTigue and Tripathi, 2008). Due to OLs increased synthesis of lipids for myelin formation, they maintain a relatively large number of peroxisomes which further contribute to increased oxidative stress and produce hydrogen peroxide as a byproduct (Dewar et al., 2003; McTigue and Tripathi, 2008). As myelin synthesis is ATP dependant, toxic byproducts of ATP synthesis, which also include hydrogen peroxide and other reactive oxygen species (ROS) from cellular metabolism, can cause OL DNA damage, lead to p53 mediated OL apoptosis, and may even have a putative role in MS pathogenesis (Ladiwala et al., 1999; Uberti et al., 1999; Mouzannar et al., 2001; Wosik et al., 2003; McTigue and Tripathi, 2008). Cells have mechanisms in place to ensure removal of ROS to prevent cellular damage. However, OLs have relatively low basal expression of glutathione, an important antioxidative enzyme that reduces ROS to prevent cellular damage, thereby making them further susceptible to ROS induced DNA damage (Thorburne and Juurlink, 1996; McTigue and Tripathi, 2008). 


Figure 4: HDAC1/2 involvement in
the DDR. This model illustrates how
HDAC1/2 activity is necessary for
efficient DNA repair. HDAC-mediated
deacetylaiton is required for NHEJ
mediated DNA repair. Compounds that
block HDAC1/2 hyper-acetylation
causing defective NHEJ repair. As
NHEJ is one of the primary
mechanisms for DSB repair, inhibition
may permit DSBs to persist (Miller et
al., 2010).

Though unique sensitivities of OPCs and OLs to oxidative stress has been and continues to be studied extensively, one critical component of the cellular responses to DNA damage is induction and successful completion of DNA repair. These repair mechanisms have been investigated extensively in cell lines, invertebrates, and low order vertebrates. However, investigations examining the DNA repair capacities of OPCs and OLs are limited. Of particular interest is whether cells of the oligodendroglial lineage have a reduced capacity to repair DNA, as they may have for ROS scavenging. The first study to demonstrate differences in DNA repair capacity between glial cell types was in response to alkylating DNA damage. These authors illustrated a four-fold increase in DNA repair efficiency following acute exposure to the alkylating agent O6-alkylguanine by astrocytes relative to OLs in rat primary astrocyte and OL cultures (LeDoux et al., 1996). The same group further demonstrated differences in mitochondrial DNA (mtDNA) repair following exposure to teratogen and alkylating agent methylnitrosurea (MNU) was reduced nearly two-fold in OPCs and OLs relative to astrocytes resulting in increased OPC and OL apoptosis (Ledoux et al., 1998). 
Taken together, these studies suggest a putative reduced capacity for OPCs and OLs to respond to increased ROS generation and repair DNA- at least in response to DNA alkylating agents- suggesting greater susceptibility to oxidative stress and DNA damage relative to other cell types. However, studies investigating whether these differences in oligodendroglial lineage cells in response to forms of exogenous DNA damaging agents that elicit other types of DNA damage (i.e. single-strand and double-strand DNA breaks), and whether this extends to differences in specific DNA repair mechanisms (i.e. base-excision repair, non-homologous end joining and/or homologous recombination) remain to be defined.

\section{CICI: SIGNIFICANCE AND PATHOPHYSIOLOGY}

\section{Chemotherapy-induced cognitive impairment $(\mathrm{CICl})$}

As a phenomenon, $\mathrm{ClCl}$ is only recently beginning to be appreciated clinically and the mechanisms of which remain largely undefined. Various studies have demonstrated that the clinical consequences of $\mathrm{ClCl}$ are typically subtle but have a broad range of detrimental effects on cognition, including but not limited to: attention, concentration, executive function, working and remote memory, and processing speed (Fig. 5) (Ahles and Saykin, 2002; Anderson-Hanley et al., 2003; Ferguson and Ahles, 2003; Tannock et al., 2004; Ahles and Saykin, 2007). Further complicating understanding of $\mathrm{ClCl}$ pathogenesis, it appears only a subgroup of chemotherapy patients present with long-term cognitive changes (Ahles and Saykin, 2007). However, according to a recently published literature 
review, $\mathrm{ClCl}$ incidence varies greatly, with the extensive variability attributed to differences in methodology and a paucity of non-conflicting longitudinal studies (Argyriou et al., 2010). Additionally, factors such as emotional distress, anxiety, depression, estrogen and testosterone levels, hormonal therapy, and fatigue may contribute to reduced cognitive function, however, in recent studies demonstrating $\mathrm{ClCl}$ these psychological factors are taken into consideration (Ahles and Saykin, 2007; Argyriou et al., 2010).

Objective visualization to uncover pathology secondary to chemotherapy that may contribute to $\mathrm{ClCl}$ onset involves magnetic resonance imaging (MRI) and positron emission tomography (PET) studies (Ahles and Saykin, 2007). Decreased volume in brain structures relevant for various cognitive functions as well as reduced integrity of white matter tracts as determined by MRI following chemotherapy have been associated with cognitive decline (Stemmer et al., 1994; Saykin et al., 2003; Ahles and Saykin, 2007). Longitudinal studies in breast cancer patients demonstrated decreased cognitive function in $61 \%$ of study participants who received adjuvant chemotherapy and altered neuropsychological testing in $33 \%$ of women who received doxorubicin/ cyclophosphamide treatment (Jansen et al., 2008; Argyriou et al., 2010). Additional studies assessing cognitive function in long-term breast cancer survivors have found reduced gray and white matter volumes across multiple brain regions including the prefrontal cortex, hippocampus, parahippocampal gyrus and precuneus, and related cognitive decline to reduction of normal appearing white matter in the genu of the corpus callosum (Inagaki et al., 2007; 
Abraham et al., 2008; Argyriou et al., 2010). Furthermore, attenuated cellular metabolism in the frontal cortex, basal ganglia, and cerebellum following chemotherapy as determined by PET scan were observed in another study of chemotherapy treated breast cancer survivors. The authors further demonstrated a correlation of altered short-term memory test performance with glucose metabolism in the inferior frontal gyrus (Argyriou et al., 2010).

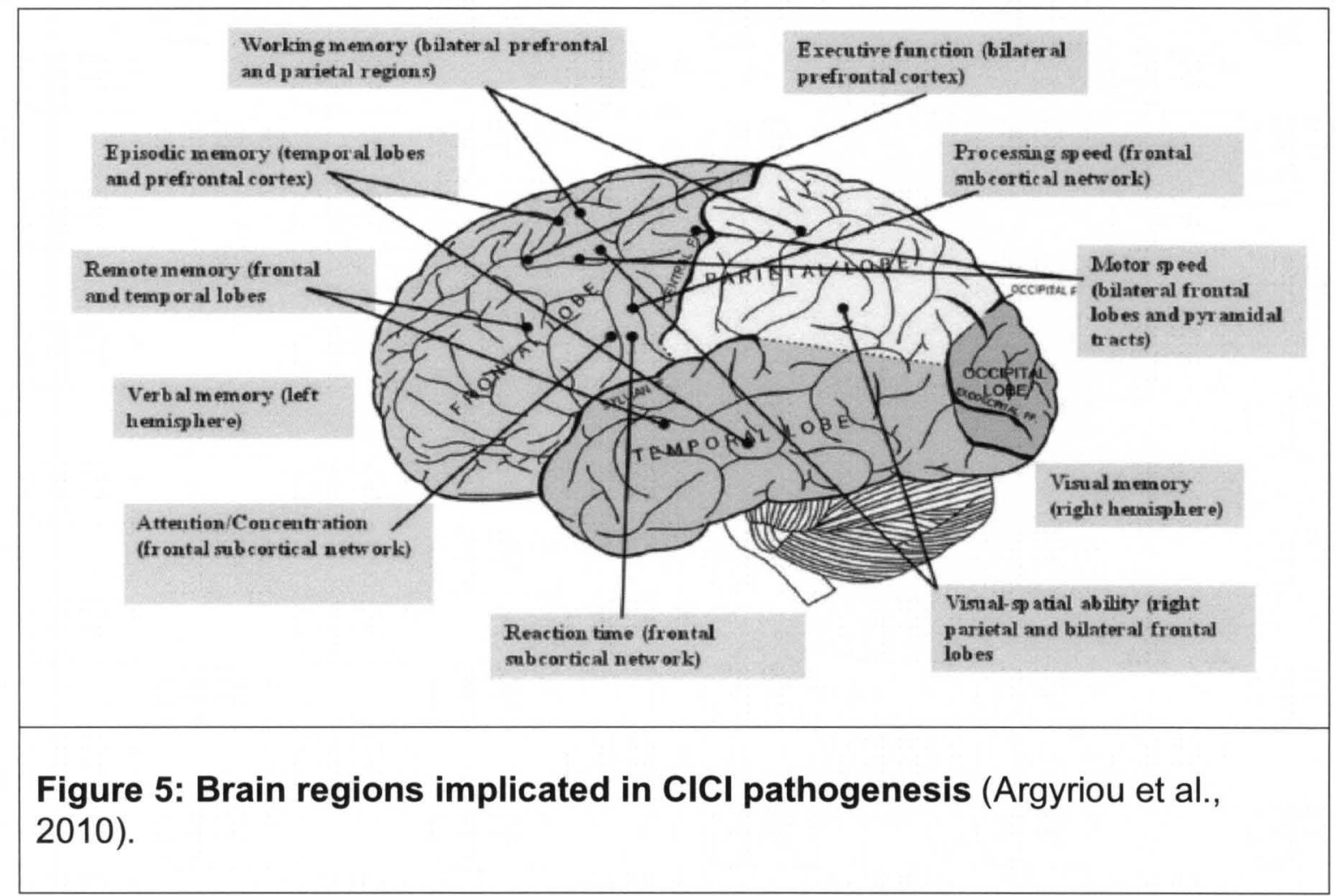

Chemotherapeutic agents such as carmustine and methotrxate have previously been demonstrated to cause leukoencephalopathy, which have characteristics of periventricular white matter lesions and cortical atrophy (Dietrich et al., 2008). In fact, several month delayed onset of white matter abnormalities are present in $70 \%$ of patients treated with high-dose chemotherapy (Stemmer et al., 1994; Brown et al., 1998; Dietrich et al., 2008). 
Lastly, delayed leukoencephalopathy syndrome as detected by MRI indicated significant white matter damage in patients largely receiving methotraxate, 5flurouracil, carmofur, and capecitabine chemotherapy treatment (Dietrich et al., 2008). Therefore, extensive imaging studies accompanied by testing of cognitive function of patients receiving a variety of chemotherapeutic treatment not only uncover putative pathological findings in $\mathrm{CICl}$ development, but also demonstrates the need to further our understanding of the mechanisms underlying $\mathrm{ClCl}$ pathogenesis as the number of chemotherapeutic agents, and the number of cancer survivors, continue to increase.

\section{Chemotherapeutic Agents: Crossing the Blood-Brain Barrier}

In order for the systemically administered chemotherapeutic agents to be toxic to the CNS, they, or there byproducts, must be blood-brain barrier (BBB) penetrable (Ahles and Saykin, 2007). Although a majority of chemotherapeutic drugs do not pass through the BBB at levels that may be effective against CNS tumors, levels as low as those having little efficacy against a peripheral tumor itself may still be found in the CNS and be sufficiently toxic towards nontransformed CNS cells (Fig. 6). A recent study demonstrated cisplatin, cytosine arabinoside, and carmustine resulted in increased cell death in multiple regions of mouse brains, including the hippocampus and the corpus callosum, at drug levels lower than needed for efficacious tumor cell death (Ahles and Saykin, 2007; Dietrich et al., 2008). Furthermore, genetic polymorphisms in genes encoding BBB drug transporters may result in increased concentration of chemotherapeutic drugs in the CNS (Ahles and Saykin, 2007). Individuals who 
are homozygous for the $C$ allele in the multidrug resistance 1 (MDR1) gene (C3435T in exon 26) which encodes P-glycoprotein (P-gp), a common substrate for many chemotherapeutic drugs and has a protective role in transporting toxic substances out of cells, have shown reduced plasma concentrations of various drugs relative to people homozygous for the T allele (Ahles and Saykin, 2007).

Additionally, alterations in blood flow dynamics may promote increased passage of chemotherapeutic agents into CNS parenchyma. Chemotherapetuic agents have been shown to reduce cerebral blood flow and may cause vascular toxicity themselves (Seigers and Fardell, 2011). Though intended to improve drug delivery to the brain, cerebral hypoperfusion was recently shown to be effective in increasing intraarterial deposition of the already lipid soluble drug carmustine into brain tissue (Joshi et al., 2008). However, such change in blood flow dynamics during chemotherapeutic treatment, whether they are an intentional manipulation of CNS blood flow or not, may be sufficient to expose healthy parts of the brain and spinal cord to increased levels of chemotherapeutic agents. Furthermore, vascular injury perhaps following ischemic or hemorrhagic stroke occurring as adverse effects of chemotherapeutic treatment (as has previously described with cisplatin) or due to co-morbidities in patients receiving chemotherapy could result in an unexpected increase in drug exposure to normal cells of the CNS as the BBB would likely be compromised (Meattini et al., 2010). Therefore, when determining the CNS toxicity potential of any chemotherapeutic agent, it is important to not only assess the BBB penetrability properties of the 
drug itself but to also consider changes in vascular flow, injury, and the integrity of the BBB in the drug's environment.

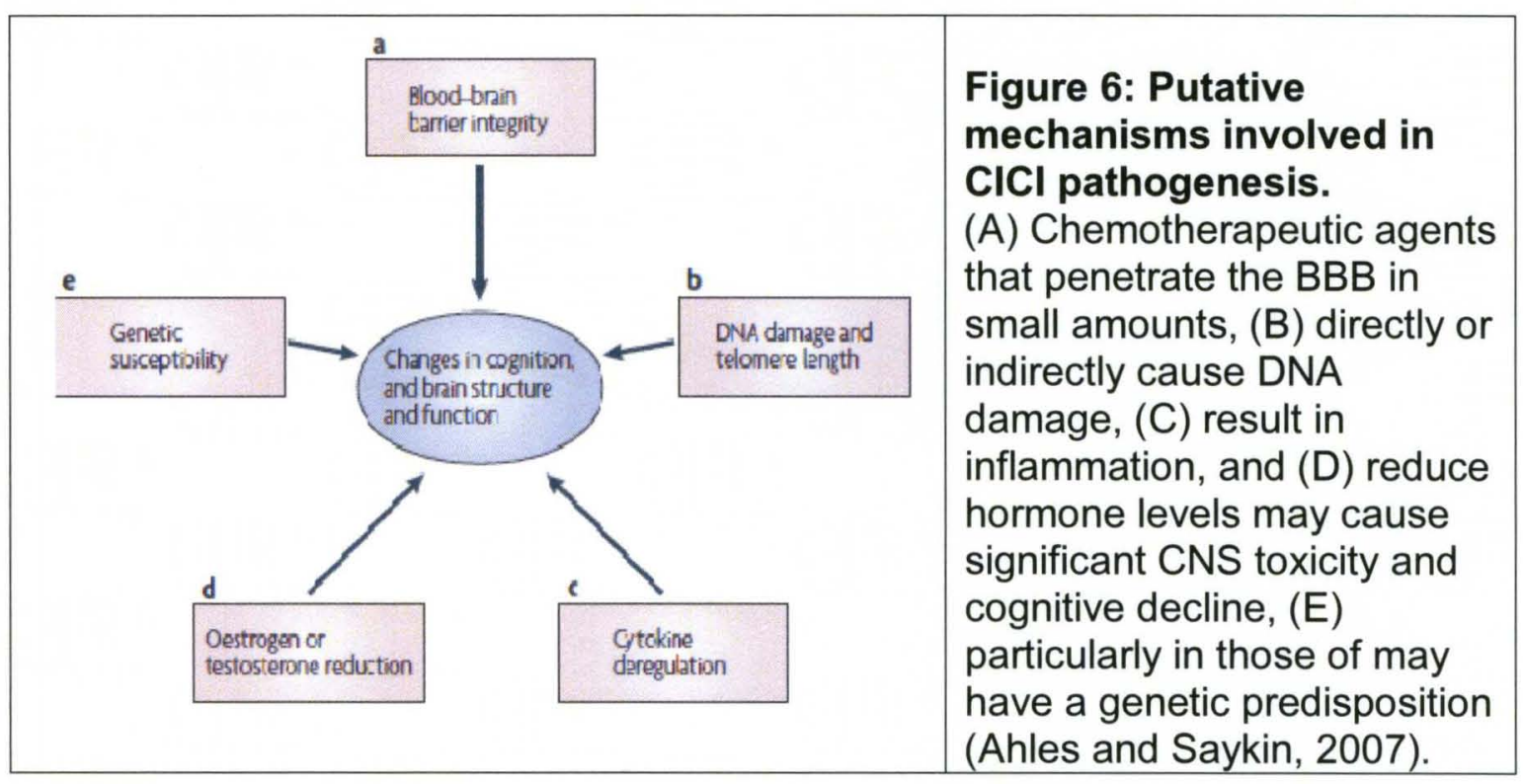

\section{$\mathrm{CICl}$ pathogenesis secondary to DNA damage}

Chemotherapy-mediated DNA damage of transformed cells is one of many mechanisms involved in transformed cell death but may be the most relevant one relative to $\mathrm{CICl}$ pathogenesis (Fig. 6). Anti-metabolites are a class of chemotherapeutic agents that disturb DNA and RNA replication and formation, respectively, by inhibiting the synthesis of new nucleic acids (Seigers and Fardell, 2011). In relation to $\mathrm{CICl}$ pathogenesis and subsequent clinical presentation, 5-fluorouracil (5-FU) and methotrexate (MTX) are the most extensively studied within this class of compounds (Janelsins et al., 2011; Seigers and Fardell, 2011). Rats displayed reduced Morris water maze (MWM) learning, novel object recognition (NOR), and operant response learning following MTX treatment, all indicators of increased cognitive impairment 
(Seigers et al., 2008). 5-FU treatment showed similar results of impaired object recognition placement and retrieval of a learned operant response, all of which is again suggestive of increased cognitive impairment (Seigers and Fardell, 2011). MTX has also demonstrated to have direct neurobiological affects that may account for the observed cognitive deficits. Increased oxidative damage, reduced neurogenesis, and increased neurotoxic apoptosis occur following MTX treatment in animals (Seigers et al., 2009; Janelsins et al., 2011). Lastly, both MTX and 5-FU systemic administration alone induced CNS toxicity is not limited to neuronal effects as significant white matter damage is also observed (Han et al., 2008; Janelsins et al., 2011; Seigers and Fardell, 2011).

Another class of chemotherapeutic drugs, the DNA alkylating agents, which damage DNA by directly adding an alkyl group to guanine, have also demonstrated to induce an appreciable amount of cognitive deficits. Cyclophosphamide (CYP) is one of the most extensively studied and displays cognitive impairment in a number of animal studies. In rats, whether alone or in combination with doxorubicin, both passive avoidance task learning and contextual fear conditioning were impaired following treatment (Seigers and Fardell, 2011). Following CYP treatment in mice, memory retention, NOR, and passive avoidance task learning were all effected suggesting development of cognitive deficits (Seigers and Fardell, 2011). Impariment of these tasks might be explained by increased oxidative stress following CYP and doxorubicin administration as antioxidant treatment with $\mathrm{N}$-acetyl cysteine improved cognitive function (Konat et al., 2008; Seigers and Fardell, 2011). Furthermore, CYP 
treatment reduced hippocampal neurogenesis in mice that displayed reduced memory retention (Yang et al., 2010). Lastly, thioTEPA, another chemotherapeutic agent in this class, displayed a long term dose-dependent reduction in hippocampal cell proliferation with accompanying memory deficits further implicating alkylating agent mediated neurotoxicity in $\mathrm{ClCl}$ pathogenesis.

Cisplatin, a platinum containing chemotherapeutic agent that acts as an alkylating agent (without alkyl group addition), resulting in DNA crosslinking, and promoting apoptosis, has also been demonstrated to cause cognitive impairment and neurotoxicity (Seigers and Fardell, 2011). Oxaliplatin, an analogue of cisplatin used in the treatment of advanced colorectal cancer, display reduced NOR and spatial reference memory. When given in combination with 5-FU, performance on these tasks worsens (Seigers and Fardell, 2011). Additionally, cisplatin resulted in in vitro cytotoxicity of cerebellar granule neurons, though astrocytes appear to be not as susceptible (Wick et al., 2004). Cisplatin treatment in the developing rat brain resulted in lesion formation in multiple brain regions including the cortex and the hippocampus. Morphologically analysis suggested neurons were undergoing excitotoxicity with subsequent active cell death (Rzeski et al., 2004). Additionally, cisplatin treatment in rats showed greater toxicity in CNS proliferating cells and mature OLs demonstrating increased cell death in the corpus callosum in addition to relevant cancer cell lines (Dietrich et al., 2006). Overall, studies involving these agents suggest direct a connection between reduced neurogenesis, and $\mathrm{OL}$ and neuronal death in a range of brain regions 
with behavioral manifestations suggestive of cognitive decline, such as memory deficits, following treatment.

Finally, inhibitors of DNA topoisomerase I and II causing the formation of single-strand breaks (SSB) and double strand breaks (DSB) in the DNA structure resulting in cycle arrest and/or apoptosis, has been shown to display neurotoxicity as well. DNA topoisomerases are involved in reducing torsional stress following unwinding of the DNA double helix during DNA replication and transcription (Seigers and Fardell, 2011). As the relaxation of torsional stress involves making DNA breaks with subsequent re-ligation, small molecule mediated topoisomerase stabilization prevents DNA reannealing resulting in DNA SSBs and DSBs. Primary cerebellar granule neurons demonstrated a 10-fold higher susceptibility to topotecan, a derivative of the topoisomerase I inhibitor camptothecin, relative to glioma cells (Wick et al., 2004). In a phase II clinical trial using a combinatorial treatment of gemcitabine with etoposide, a topoisomerase II inhibitor, caused a significant increase in cognitive impairment relative to another treatment group not receiving etoposide (Mok et al., 2005). Lastly, in addition to DNA DSB formation with subsequent neuronal apoptosis, etoposide mediated nucleolar stress resulted in non-apoptotic neurotoxicity of primary rat cortical neurons potentially providing a critical mechanistic clue to the clinical manifestations of $\mathrm{ClCl}$ as mediated by topoisomerase II inhibitors (Pietrzak et al., 2011). 


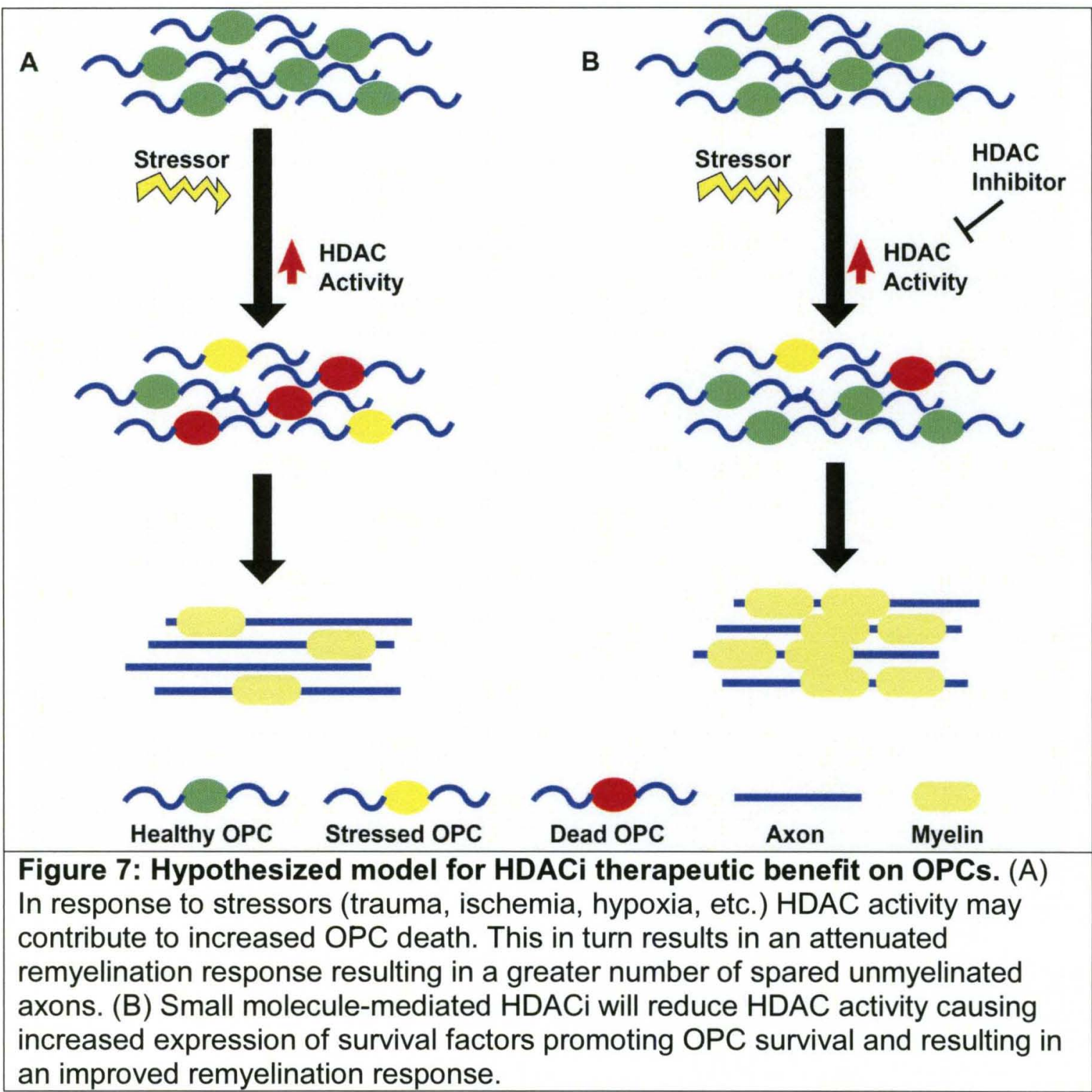

\section{SUMMARY}

\section{Putative Model}

Despite concerns regarding inhibition of OPC maturation following $\mathrm{HDACi}$, we wanted to determine whether transient treatment of OPCs with a small molecule HDAC inhibitor may be a viable therapy, as has been suggested in other CNS disease models and cell types, to promote OPC survival in acutely stressed conditions (Fig. 7). To investigate this, we developed and characterized 
a purified postnatal mouse cortical OPC primary culture as discussed in chapter two. As presented in chapter three however, rather than cell survival, extensive cell death was observed acutely following treatment in vitro, as well as in parallel in vivo studies. Lastly in chapter four it is demonstrated that putative mechanisms involved in this OPC susceptibility to HDACi-mediated cytotoxicity, such as DNA damage, are similar to those already described in transformed cell lines.

Overall, these results suggest our original hypothesis of a therapeutic effect on OPC survival following small molecule mediated HDACi in the presence of other stressors was incorrect. Rather, HDACi is detrimental to OPC survival demonstrating that great caution must be taken in the future clinical use and development of HDAC inhibitors due to potential effects of OPC toxicity, including but not limited to DNA damage induction. Subsequent dysmyelination may occur that may lead to cognitive deficits similar to those described in $\mathrm{CICl}$ pathogenesis following treatment with other well investigated chemotherapeutic agents. 


\section{CHAPTER 2}

\section{ISOLATION OF CORTICALLY DERIVED MOUSE OLIGODENDROCYTE PRECURSOR CELLS}

\section{INTRODUCTION}

The developing mammal maintains an extensive population of CNS progenitor cells maturing along the oligodendroglial lineage to yield fully differentiated OLs that myelinate all axons within the CNS (Wolswijk and Noble, 1989; Noble et al., 1992). Of these progenitor populations, OPCs provide the largest source of maturing OLs, though a significant OPC subpopulation avoids differentiation maintaining their proliferative state (Nishiyama et al., 2009; Trotter et al., 2010). Isolation and subsequent in vitro study of OPCs both in the developing and adult mammal offers critical insights into putative disease and injury mechanisms involved in demyelinating disease states such as multiple sclerosis and CNS white matter infarctions as observed in ischemic stroke (Nishiyama, 1998). Furthermore, OPC engraftment into the injured CNS to replenish lost OLs continues to offer promise as a cell therapeutic method for the treatment of spinal cord injury (Cao et al., 2010). 
A variety of cell surface markers indicate the state of OPC maturation in the oligodendroglial lineage. A2B5, a cell surface glycoside, and NG2, a chondroitin sulfate proteoglycan, are indicative of the immature OPC population (Nishiyama et al., 2009). However, $\mathrm{NG}^{+}$cells may not be specific to cell progenitors committed to an OL fate. In addition to having recently described synaptic associations to neurons, they also maintain the capacity to terminally differentiate into astrocytes in addition to OLs (McTigue and Tripathi, 2008; Emery, 2010). O4, a later stage OPC-specific galactocereboside found on the cell surface, contrasts A2B5 and NG2 in serving as a more reliable marker for OPCs committed to an oligodendroglial fate (Nishiyama, 1998; Nicolay et al., 2007; Nishiyama et al., 2009; Trotter et al., 2010). 01, another oligodendroglial lineage specific galactocereboside expressed on the cell surface of premyelinating OLs is indicative of an OPC that no longer maintains its proliferative potential but is transitioning to the terminally differentiated OL. As the premyelinating $\mathrm{OL}$ matures into the myelinating $\mathrm{OL}$, the presence of the $\mathrm{O} 1$ cell surface antigen is accompanied by myelin basic protein (MBP) expression, one of the primary protein constituents of myelin and a marker for the mature myelinating OL (Nicolay et al., 2007; Trotter et al., 2010).

Numerous oligodendroglial cell lines have been developed for the study of OL function (Lin et al., 2006; Mock et al., 2006; Pringproa et al., 2008). However, there is considerable variability between these cell lines largely depending on the source (i.e. chemically induced rodent tumors or spontaneous human tumors), isolation method, and growth conditions. Often times, these lines maintain 
considerable proliferative potential, even once engrafted (a hallmark of their potential tumorigenicity), and may prove difficult for induction of differentiation (Lin et al., 2006; Pringproa et al., 2008). OPC cultures derived from the formation of neurospheres or oligospheres yield similar variability in genetic and phenotypic makeup resulting in considerable departure from their in vivo counterparts (Chen et al., 2007b; Zhao et al., 2010). Furthermore, with passage, subtle cellular changes occur resulting in a molecularly different cell type than what was originally isolated (Lin et al., 2006). Lastly, nearly all existing primary OPC cultures from rodent models rely on more premature markers A2B5 and NG2 which may not have as extensive oligodendroglial lineage commitment (Larsen and Yong, 2004; Hamanoue et al., 2009). Primary OPC cultures will be better served using a later stage marker than is more limited to the oligodendroglial lineage but still maintain their proliferative capacity.

To address these concerns in existing OPC isolation methods, we developed and characterized a reliable and reproducible primary culture of cortically-derived OPCs from mouse pups. We limited the scope of this characterization to no more than a single passage following each OPC isolation to limit the potential of genetic and phenotypic drift often observed with additional passages. Furthermore, we used the OPC cell surface antigen $\mathrm{O} 4$, meeting the criteria of isolating OPCs that are proliferative and have greater commitment to OL differentiation than the oligopotent A2B5 and NG2 progenitor cells. Though differentiation of these isolated OPCs in vitro still yields both OLs and astrocytes, isolated mouse OPC/ DRG co-cultures results in extensive OPC maturation with 
nearly complete absence of astrocytes. Overall this method will facilitate the continued study of OPC differentiation and OL function and provide in vitro findings that will be better recapitulated in vivo.

\section{MATERIALS AND METHODS}

\section{Animals}

E12-E15 time pregnant C57BI/6 mice and E15 Sprague-Dawley rats were purchased from Charles River Laboratories (Wilmington, MA). All animal procedures were performed according to the guidelines of University of Louisville Institutional Animal Care and Use Committee protocols and the National Institutes of Health.

\section{Preparation for mouse OPC isolation}

Prior to OPC isolation, $13.5 \mu \mathrm{l}$ of $50 \mathrm{mM} \beta$-mercaptoethanol was combined (Sigma, St. Louis, MO) to $10 \mathrm{ml}$ of Solution 2 from the Neural Tissue Dissociation Kit (Miltenyi Biotec, Bergisch Gladbach, Germany). MACS Buffer was prepared by adding $5 \%$ bovine serum albumin (BSA), $0.5 \mathrm{M}$ EDTA, and $5 \mu \mathrm{g} / \mathrm{ml}$ insulin in Dulbecco's phosphate buffered saline supplemented with $1 \mathrm{~g} / \mathrm{L}$ glucose (all from Sigma). OPC-A media was prepared by adding $2.1 \mathrm{~g} / \mathrm{L} \mathrm{NaHCO}{ }_{3}($ Sigma) to dissolved DMEM-F12 without HEPES powder (Invitrogen, Carlsbad, CA). N2 supplement (1\%), B27 supplement (2\%), Penicillin/Streptomycin (1\%, all from Invitrogen), BSA (0.01\%, Sigma), $40 \mathrm{ng} / \mathrm{ml}$ fibroblast growth factor 2 (FGF2, Millipore, Billerica, MA), and $20 \mathrm{ng} / \mathrm{ml}$ platelet-derived growth factor-aa (PDGFaa, Sigma) was added to the previously prepared DMEM-F12. Differentiation media 
components included N2 supplement (1\%), B27 supplement (2\%),

Penicillin/Streptomycin (1\%), $50 \mu \mathrm{g} / \mathrm{ml}$ insulin, and $40 \mathrm{ng} / \mathrm{ml}$ triiodo-thyronine (T3, Sigma) in DMEM/F12. Differentiation media was supplemented with $1 \mathrm{ng} / \mathrm{ml}$ ciliary neurotrophic factor (CNTF, Sigma) where appropriate. Where described, "pre-equilibration" refers to cell media incubated for a minimum of 30 minutes in $37^{\circ} \mathrm{C}, 5 \% \mathrm{CO}_{2}$ prior to use.

\section{Dissection of mouse cortices}

Whole mouse brains were harvested from ice-anesthetized postnatal day 5-7 mouse pups by decapitation following loss of reflexive movement (approximately 5-8 minutes in ice). The skin and skull were cut along the midline and reflected to expose the brain. The brain was then removed and placed in a pre-chilled Petri-dish with sufficient volume of $\mathrm{HBSS}$ without $\mathrm{Ca}^{+}$and $\mathrm{Mg}^{+}$ (Lonza, Walkersville, MD) to submerge the brain. Afterwards, the cerebellum, brain stem, olfactory bulbs, and olfactory tracts were removed, and followed by careful dissection of the meninges from the remaining cortex. This was repeated for as many brains as desired for a particular isolation. Finally, dissected cortices were transferred into a pre-weighed dish with pre-chilled $\mathrm{HBSS}$ without $\mathrm{Ca}^{+}$and $\mathrm{Mg}^{+}$and all cortices weighed.

\section{Tissue dissociation of mouse cortices}

Using the Neural Tissue Dissociation Kit (Miltenyi Biotec), an appropriate volume of Enzyme Mix 1 corresponding to the cortical tissue mass was prepared. For every $400 \mathrm{mg}$ of tissue, $50 \mu \mathrm{l}$ of Solution 1 was added to $1.9 \mathrm{ml}$ of pre-made Solution 2 with BME. This was briefly vortexed and then preheated in 
a $37^{\circ} \mathrm{C}$ water bath for 10 minutes before use. As Enzyme Mix 1 was heating, 3 Pasteur pipettes were fire polished with decreasing tip diameters and set aside for later use. Brains were transferred to a $0.5 \mathrm{ml}$ drop of HBSS in the middle of a fresh $10 \mathrm{~cm}$ dish. Cortices were diced thoroughly $\left(1 \mathrm{~mm}^{3}\right.$ pieces) with a razor blade, then 5-6 ml of HBSS was added then tissue pippetted into a $15 \mathrm{ml}$ conical tube. The dish was rinsed well with fresh HBSS to collect any remaining tissue and added into the $15 \mathrm{ml}$ conical tube. Tissue was centrifuged at $300 \times \mathrm{g}$ for 2 minutes at room temperature (RT). Supernatant was then carefully aspirated, followed by addition of the pre-heated Enzyme Mix 1 to the pellet, and then gently mixed taking care to avoid air bubbles. Tissue was then incubated for 15 minutes in the $37^{\circ} \mathrm{C}$ water bath, inverting the tube several times every 5 minutes to resuspend settled cells. During incubation, an appropriate volume of Enzyme Mix 2 was prepared. For every $400 \mathrm{mg}$ of tissue, $20 \mu \mathrm{l}$ of Solution 3 was added to $10 \mu$ of Solution 4 . Once the incubation was complete, Enzyme Mix 2 was added to the tube containing the tissue. This was inverted gently to mix and then slowly triturated approximately 15 times using the widest fire-polished pipette again taking care to avoid air bubbles. This was then incubated in a water bath for 10 minutes, inverting several times after 5 minutes of incubation. Tissue was slowly triturated approximately 15-20 times each using the remaining two firepolished pipettes in decreasing diameter. Then tissue was incubated again in a water bath for 10 minutes, inverting several times after 5 minutes of incubation. During the incubation, a $40 \mu \mathrm{m}$ cell strainer was pre-wetted with $5 \mathrm{ml}$ DPBS into a $50 \mathrm{ml}$ conical tube. DPBS was then aspirated from the tube and following the 
tissue incubation, the suspension was slowly decanted through the strainer. The $15 \mathrm{ml}$ tube with the tissue was rinsed with $10 \mathrm{ml}$ of $\mathrm{HBSS}$ with $\mathrm{Ca}^{+}$and $\mathrm{Mg}^{+}$ (Invitrogen) and added this to the strainer. The cell suspension was centrifuged at $300 \times g$ for 10 minutes at RT. The supernatant was gently aspirated, leaving approximately $2-5 \mathrm{ml}$ of supernatant in the tube, and cells were washed with 10 $\mathrm{ml}$ of HBSS with $\mathrm{Ca}^{+}$and $\mathrm{Mg}^{+}$and centrifuged $300 \times g$ for 10 minutes at RT. The supernatant was aspirated and the pellet gently resuspended in $5 \mathrm{ml}$ MACS buffer. A small aliquot was then removed for a cell count and the cell suspension transferred to a $15 \mathrm{ml}$ tube and centrifuged $300 \times \mathrm{g}$ for 5 minutes. During centrifugation, the total number of live cells were counted by Trypan Blue visualization. Depending on the number of brains dissected, counts ranged from $1.0-5.0 \times 10^{7}$ cells with a cell viability range of $85-95 \%$.

\section{Magnetic cell sorting (MACS) for OPC enrichment}

Following the total cell count, the supernatant was gently aspirated and rat anti-mouse IgM magnetic beads ( $10 \% \mathrm{v} / \mathrm{v}$ in MACS Buffer, Miltenyi Biotec) were added to the cells. The pellet was then gently resuspended and incubated for 15 minutes at $4^{\circ} \mathrm{C}$ with gentle shaking. As cells may settle, cells were mixed by tapping the tube every 5 minutes. Following incubation, cells were resuspended in $5 \mathrm{ml}$ MACS buffer and centrifuged at $300 \times g$ for 5 minutes at RT. During centrifugation, we set up a MACS MS column (Miltenyi Biotec) on a MACS magnetic stand (Miltenyi Biotec) and equilibrated the column with $0.5 \mathrm{ml}$ of MACS buffer. A $15 \mathrm{ml}$ conical tube was then positioned below the column to collect the flow-through. Following the spin, cells were resuspended in $0.5 \mathrm{ml}$ 
MACS buffer, transferred to the column, and the column was washed four times with $0.5 \mathrm{ml}$ MACS buffer. The initial flow through and all subsequent washes were collected in a single $15 \mathrm{ml}$ tube and centrifuged at $300 \times g$ for 5 minutes. Following centrifugation, the cell pellet was resuspended in $400 \mu$ l of diluted $\mathrm{O} 4$ antibody. This was incubated $5-10$ minutes at $4^{\circ} \mathrm{C}$ with gentle shaking and mixed once by tapping. The cell suspension was then washed two times by adding $5 \mathrm{ml}$ MACS buffer followed by centrifugation at $300 \times g$ for 5 minutes. During centrifugation, an additional MACS rat anti-mouse IgM beads mix was prepared based on the total cell count. For every $1 \times 10^{7}$ cells, $20 \mu$ MACS beads were mixed with $180 \mu \mathrm{l}$ MACS Buffer. Following centrifugation, cells were resuspended in the second MACS beads mix and incubated 15 minutes at $4^{\circ} \mathrm{C}$ with gentle shaking. If cells settled, the suspension was mixed by tapping every 5 minutes. Afterwards, cells were resuspended in $5 \mathrm{ml}$ MACS buffer and centrifuged $300 \times g$ for 5 minutes. During centrifugation, another MACS column was equilibrated with $0.5 \mathrm{ml}$ MACS buffer as described above. The pellet was resuspended in $0.5 \mathrm{ml}$ MACS buffer and added to the column, washed four times with $0.5 \mathrm{ml}$ MACS buffer, and bound cells eluted in $1 \mathrm{ml}$ MACS buffer into a fresh $15 \mathrm{ml}$ tube. Air bubbles were unavoidable during this step; however, we minimized their contact with the cell suspension at the bottom of the tube. A fresh column was quickly equilibrated, eluted cells added, the column washed four times with $0.5 \mathrm{ml}$ MACS buffer, and cells eluted into a fresh $15 \mathrm{ml}$ tube in $1 \mathrm{ml}$ pre-equilibrated OPC-A medium. $1 \mathrm{ml}$ of additional medium was added to this cell suspension and mixed thoroughly. Cells were counted, plated in a PDL/laminin-coated $10 \mathrm{~cm}$ tissue 
culture dish, and incubated in $37^{\circ} \mathrm{C}, 5 \% \mathrm{CO}_{2}$. Approximately $9,000-15,000$ cells $/ \mathrm{cm}^{2}$ were plated for a successful OPC isolation.

\section{OPC expansion, differentiation, and OPC/DRG co-culture}

The day following OPC isolation, all medium was removed from the plate and replaced with fresh, pre-equilibrated OPC-A media. For subsequent daily medium changes, $1 / 2$ medium was removed from the culture dish and replaced with pre-equilibrated OPC-A media. Cells were then ready to passage within 6-9 days, depending on plating density and proliferative rate. Passaging was performed using Accutase ${ }^{\circledR}$ (Sigma) according to the manufacturer's instructions. Briefly, media was removed and plated OPCs were rinsed once with DPBS. Immediately afterwards, Accutase $®$ was added (approximately $1 \mathrm{ml} / 10$ $\mathrm{cm}^{2}$ ) and incubated in $37^{\circ} \mathrm{C}$ for 10 minutes. Following incubation, about half of the Accutase $\AA$ containing cells was removed and the plate rinsed 2-3 times to remove remaining adherent cells. The cell suspension was transferred to a sterile tube, the plate washed with 3-4 ml of OPC-A media, and added to the cell suspension. Immediately cells were centrifuged $300 \times g$ for 5 minutes at RT. The supernatant was gently aspirated and cells resuspended in $2 \mathrm{ml}$ of preequilibrated OPC-A media. Ideal plating densities of P1 OPCs typically ranged between $7,500-10,000$ cells $/ \mathrm{cm}^{2}$. Following passage, OPCs were fed with $1 / 2$ media change with pre-equilibrated mOPC-A every other day until used for experimentation.

For OPC differentiation, P1 OPCs were allowed to expand in OPC-A media for 3-5 days then replaced with pre-equilibrated Differentiation media. 
Depending on desired state of maturation, OPCs were allowed to differentiate in Differentiation media for 3-10 days with $1 / 2$ media change with pre-equilibrated Differentiation media every other day. For OPC/DRG co-cultures, DRGs were isolated from E15 Sprague-Dawley rat embryos as previously described (Plant et al., 2002; Cheng et al., 2007; Cao et al., 2010). Following isolation, DRGs were plated 5,000 cells $/ \mathrm{cm}^{2}$ and maintained on collagen coated coverslips in NB1 neurobasal medium to permit neurite outgrowth for at least three weeks and no more than four weeks prior to seeding with P1 OPCs. Once seeded, OPC/DRG co-cultures were maintained 7-14 days in Differentiation media without Penicillin/Streptomycin to permit OPC maturation with $1 / 2$ media change every other day using pre-equilibrated media.

\section{Immunocytochemical analyses}

Immunocytochemical analysis of OPC cultures in OPC-A and Differentiation media and OPC/DRG co-cultures were done as previously described (Cao et al., 2010). Briefly, cells were washed in DPBS and then incubated with the respective hybridoma supernatants for 45 minutes at $4^{\circ} \mathrm{C}$. Afterwards, cells were fixed in $4 \%$ paraformaldehyde at room temperature for 10 minutes. Cells were blocked and incubated overnight with additional nonhybridoma primary antibodies in $4^{\circ} \mathrm{C}$ with $10 \%$ normal donkey serum (NDS, Jackson ImmunoResearch, West Grove, PA), 0.5\% BSA (Sigma), and 0.1\% Triton X-100 in DPBS. The following day, cells were incubated in secondary antibody at room temperature for one hour in $5 \%$ NDS, $0.5 \%$ BSA, and $0.1 \%$ Triton X-100 in DPBS. Antibodies used include mouse anti-A2B5 IgM, mouse 
anti-O4 IgM, and mouse anti-O1 IgM (hybridoma derived, $70 \% \mathrm{v} / \mathrm{v}$ in $20 \%$ DPBS, and $10 \%$ NDS), rabbit anti-NG2 (1:500, Millipore), rabbit anti- $\beta$ III-tubulin (1:2000, Covance, Princeton, NJ), rat anti-MBP (1:100, Millipore), rabbit anti-GFAP (1:500, Dako, Glostrup, Denmark), and rabbit anti-Map2 (1:100, Sigma). Cells were incubated with FITC, Texas Red, and Rhodamine Red conjugated F(ab') fragment antibodies (Jackson Immunoresearch). Negative controls for antibody staining included the appropriate species-specific non-immune IgG or IgM antibodies substituted for the primary antibodies. 10X and 20X photomicrographs were captured with a Nikon TiE 300 inverted microscope equipped with a DXM1200C coded digital camera and NIS Elements software (Nikon, Melville, NY). All quantitative data are presented as means +/- S.D.

\section{RESULTS}

\section{OPC enrichment}

Using postnatal day 5-7 mouse cortices, we achieve a yield of approximately $3.7 \times 10^{5}$ cortical OPCs/brain (Fig. 8). In contrast to the previous adult rat OPC spinal cord primary culture developed in our lab, expanding the $\mathrm{O}^{+} \mathrm{OPC}$ population proved difficult. From 3 days post plating, enriched OPCs are phase bright with typical bipolar morphology (Fig. 9A). A large portion of the isolated OPC population at $\mathrm{P0}$ is $\mathrm{O}^{+}$(Fig. 9A-C). Furthermore, at $\mathrm{P} 1$ our primary culture remained almost entirely $\mathrm{A}_{2} \mathrm{~B}^{+}$and $\mathrm{NG}^{+}$(Fig. 9D-F), demonstrating an expanded population of enriched OPCs positive for early OPC markers.

Furthermore, all cells at P0 and P1 were GFAP (Fig. 9B-C) and $\beta$-III tubulin (data 
not shown) negative indicating enrichment of a cell population devoid of astrocyte and neuronal contamination, respectively.

\begin{tabular}{|l|l|l|}
\hline \multicolumn{2}{|c|}{ OPC Isolation } & $\begin{array}{l}\text { Figure 8: Cortical OPC yield } \\
\text { from mouse P5-P7 pups. }\end{array}$ \\
\cline { 1 - 2 } Yield (OPCs/Brain) & $3.68 \times 10^{5}+/-9.17 \times 10^{4}$ & \\
\hline
\end{tabular}

Following passage, despite being $\mathrm{A}_{2} \mathrm{~B}^{+}$and $\mathrm{NG}^{+}$, the percentage of $\mathrm{O}_{4}^{+}$ cells declined from approximately $80 \%$ just prior to passage (data not shown) to just under $50 \%$ of the total cell population (Fig. 10). Nonetheless, over $90 \%$ of all cells remained both $\mathrm{A}_{2} \mathrm{~B}^{+}$and $\mathrm{NG}^{+}$with continued expansion (Fig. 10). Further quantification of revealed that the isolated OPCs remained immature as they were not $\mathrm{O}^{+} \mathrm{OLs}$ and did not have any GFAP ${ }^{+}$astrocytes (Fig. 10). These data suggest with continued expansion in vitro $\mathrm{O} 4$ surface expression declines but other markers indicative of OPC phenotypes remain positive indicating maintenance of the OPC phenotype.

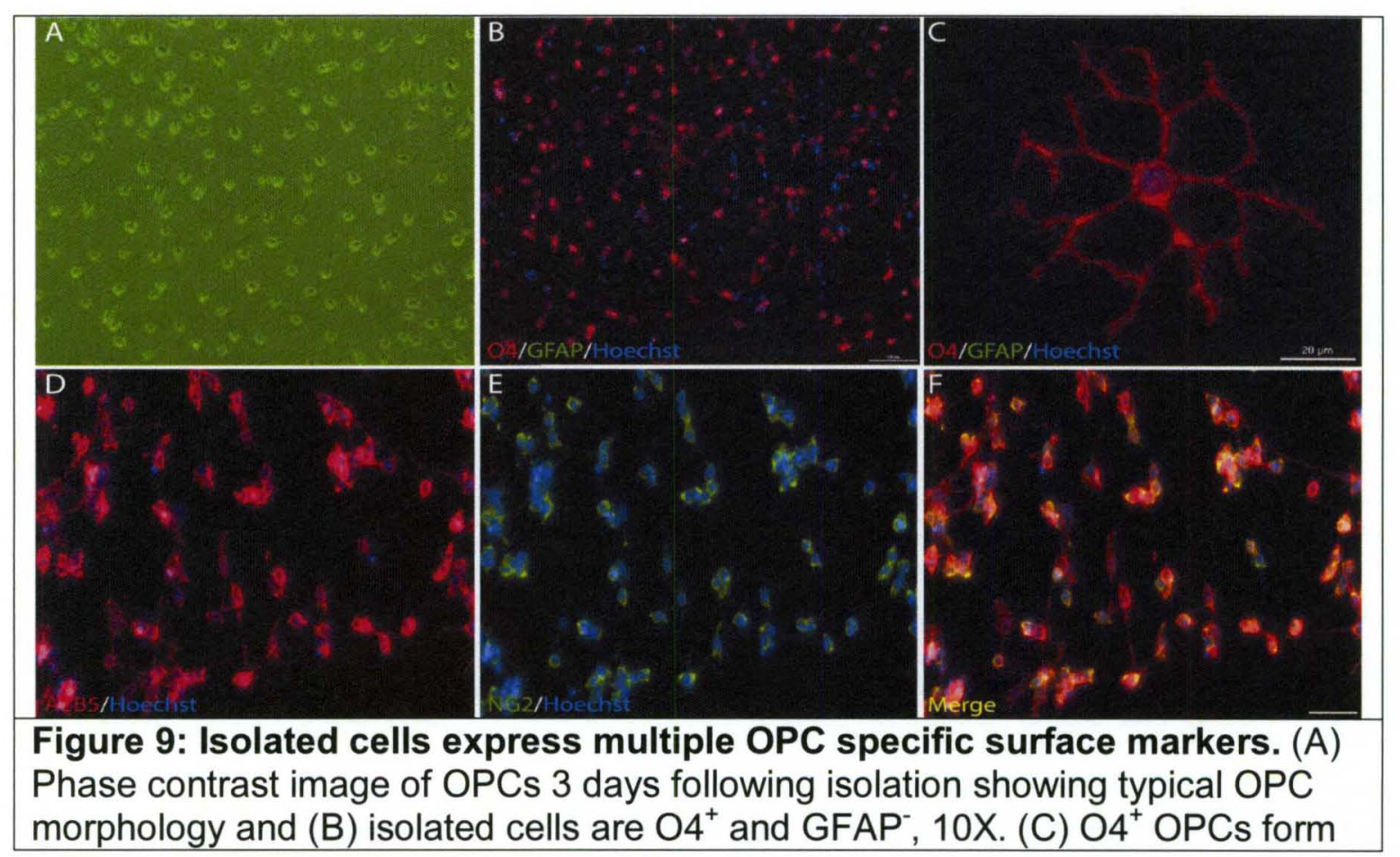


multiple processes following plating, scale bar $20 \mu \mathrm{m}$ (D-F) Following passage, P1 OPCs co-express early OPC surface markers A2B5 and NG2, 20X.

\section{OPC differentiation}

Mouse cortically derived OPC differentiation may commit to either $\mathrm{GFAP}^{+}$ astrocytes or OLs (Fig. 11). OPC differentiation from 4 to 6 days in the presence of serum and the absence of PDGFaa and FGF2 leads to astrocyte differentiation as evidenced by nearly $100 \%$ GFAP $^{+}$immunoreactivity with minimal $\mathrm{O} 4$ and $\mathrm{O} 1$ immunoreactivity (data not shown).

\begin{tabular}{|l|l|l|l|}
\hline \multicolumn{2}{|c|}{ Mouse Cortical OPC Isolation Cell Counts } & $\begin{array}{l}\text { Figure 10: P1 OPC cell counts } \\
\text { show an enriched population of } \\
\text { OPCs. Approximately } 50 \% \text { of } \mathrm{P} 1\end{array}$ \\
OPCs are $\mathrm{O}^{+}(49.05+/-6.08 \%)$ and \\
Over $90 \%$ of cells are both $\mathrm{NG} 2^{+}$ \\
$(90.14+/-6.05 \%)$ and $\mathrm{A} 2 \mathrm{~B}^{+}(94.55$ \\
\\
\hline
\end{tabular}

When OPC growth media is replaced by low serum media without PDGFaa and FGF2 but in the presence of thyroid hormone (T3) differentiation into a mixed glial population occurs. There is reduced number of cells that are phase bright following 4 days of differentiation (Fig. 11A). Nearly $40 \%$ of all OPCs form a dramatically more complex arborization pattern of $\mathrm{O}^{+} \mathrm{OLs}$ (Fig. 11B, 12). This coincides with the approximately one-third $\mathrm{O}^{+}$population of differentiated OPCs with a comparatively complex arborization (Fig. 11C, 12). However, this is accompanied by differentiation of nearly one-third of OPCs to $\mathrm{GFAP}^{+}$astrocytes (Fig. 11D, 12). OPCs differentiated into OLs also 
demonstrated myelin basic protein (MBP) co-immunostaining with $\mathrm{O}^{+} \mathrm{OLs}$ in the presence of our standard Differentiation media, though mature OLs were in the minority (Fig. 11E-G).
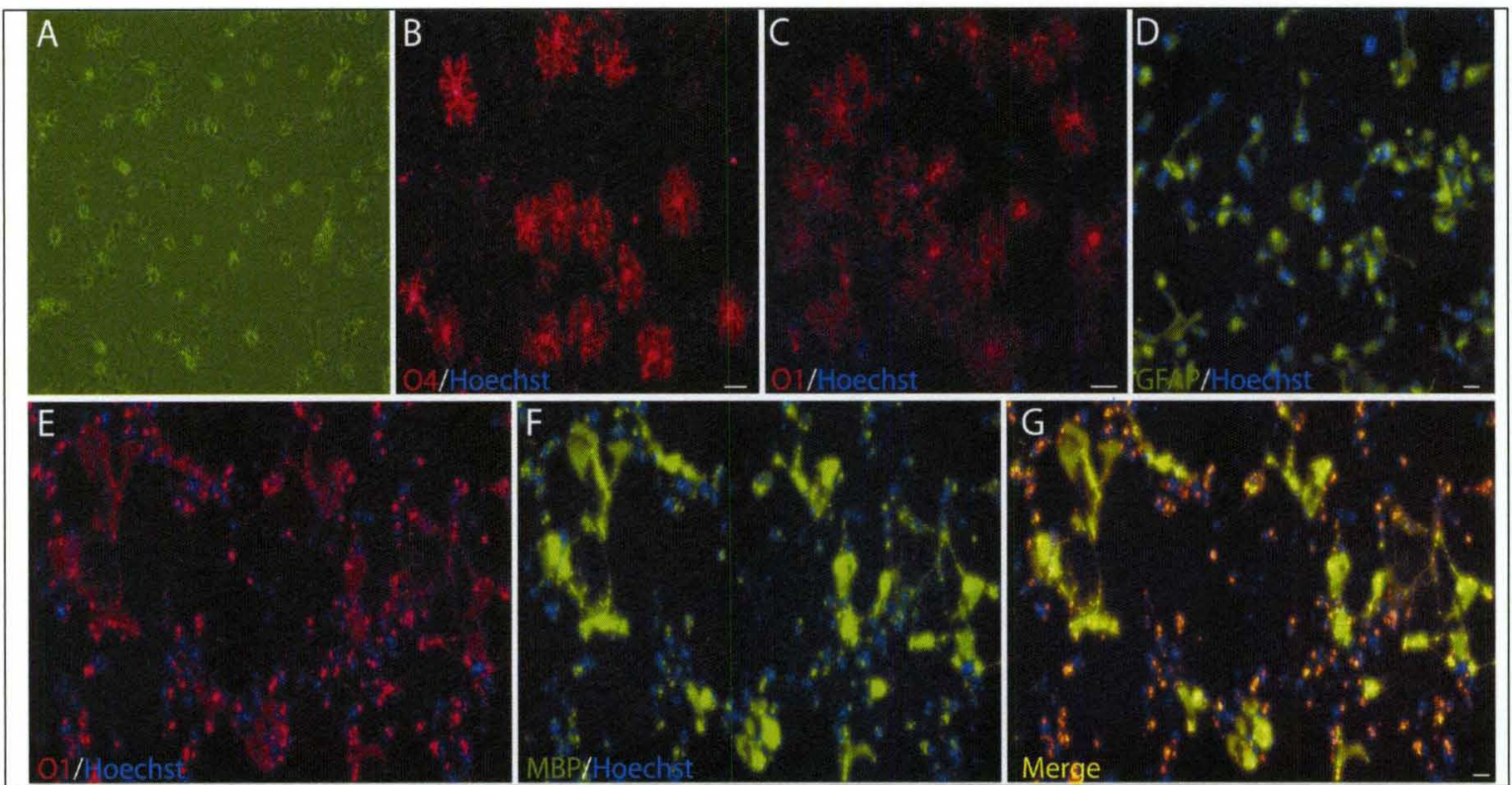

Figure 11: OPC differentiation into mature OLs. (A) 4 days following initiation of OPC differentiation, cells are less phase bright and form more processes, 10X. (B-D) Following 7 days of incubation in differentiation media, OLs are highly arborized and are $\mathrm{O}^{+}$and $\mathrm{O}^{+}$. However, there is astrocyte formation as well evidenced by $\mathrm{GFAP}^{+}$cells, 10X. (E-G) $\mathrm{O}^{+}$OLs are also $\mathrm{MBP}^{+}$indicating near full maturation and beginning of myelin synthesis, 10X.

\section{OPC/Dorsal root ganglia (DRG) co-culture}

When co-cultured with rat embryonic day 15 DRGs, cortically isolated OPCs consistently aligned with DRG neurites and differentiated into mature OLs (Fig. 13). Following 7 days of incubation, co-cultures were A2B5 and NG2 negative indicating maturation from their precursor phenotype (data not shown). Furthermore, OPCs differentiated into OLs as demonstrated by extensive $\mathrm{O}^{+}$ and $\mathrm{O}^{+}$immunostaining of complex and highly arborized OLs (Fig. 13A-F). DRG neurite-aligned OLs were also $\mathrm{MBP}^{+}$suggesting myelin synthesis along DRG 
axons (Fig. 13G-I). O4, O1, and MBP expression was maintained in co-cultures following 14 days of incubation, although there did not appear to be a significant difference in staining intensity with any of the three markers compared to cocultures following 7 days of incubation (data not shown).

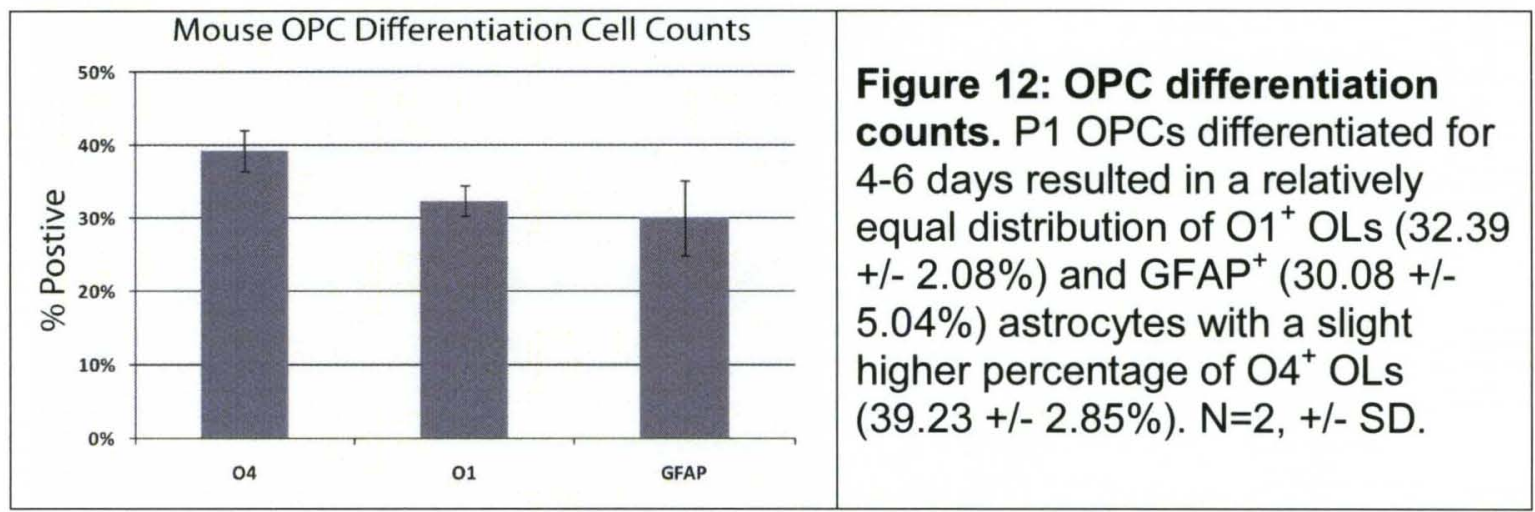

Due to reduced OPC- derived astrocyte differentiation in OPC/DRG cocultures, we next differentiated OPCs in the absence of DRGs with conditioned media (CM) collected from 7 day incubated co-cultures (Fig. 14). Following 7 days of incubation, OL maturation comparable to cultures without co-culture $\mathrm{CM}$ (Fig. 11) was observed as evident in the increased $\mathrm{O}^{+}$and $\mathrm{MBP}^{+} \mathrm{OLs}$ (Fig. 14BD). However, GFAP ${ }^{+}$astrocytes were still present as observed with standard Differentiation media (Fig. 14A).

In vitro OPC differentiation leads to a dramatic increase cell death (unpublished observations). We previously demonstrated ciliary neurotrophic factor (CNTF) improved adult rat spinal cord derived OPC survival both in vitro, upon transition to differentiation media, and in vivo following engraftment into the injured spinal cord (Cheng et al., 2007). Differentiation media supplementation with $1 \mathrm{ng} / \mathrm{ml}$ CNTF improved survival of mouse OPCs as well (data not shown). 


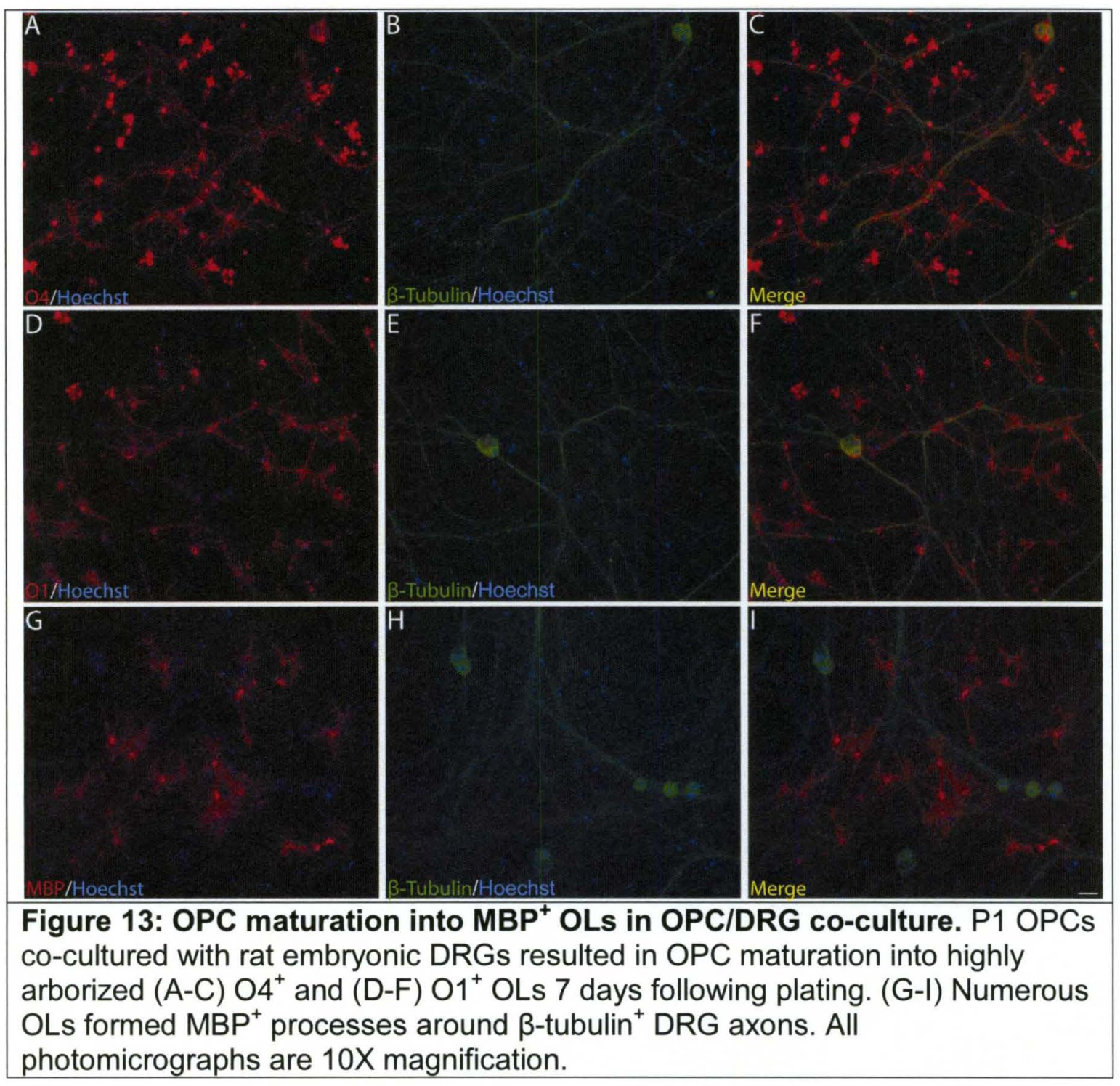

Therefore, $1 \mathrm{ng} / \mathrm{ml}$ CNTF was also added to OPCs differentiating coculture $\mathrm{CM}$ to determine if increased OPC survivability during the early phase of OPC maturation will preferentially increase the number of OPCs differentiating into OLs (Fig. 14). Following 7 days of incubation, highly complex and mature OLs that were $\mathrm{O}^{+}, \mathrm{O}^{+}$, and $\mathrm{MBP}^{+}$had formed (Fig. 14E-H). The degree with which mature OLs encompassed large areas of $\mathrm{MBP}^{+}$immunostaining strongly suggests myelin synthesis (Fig. 14G-H). This extent of OPC maturation was not 
previously seen and can only achieved with CNTF supplementation. However, OLs were also accompanied by OPC differentiation into $\mathrm{GFAP}^{+}$astrocytes revealing the continued bi-potentiality of cortically derived postnatal OPC differentiation in vitro, with or without CNTF supplementation (Fig. 14E).

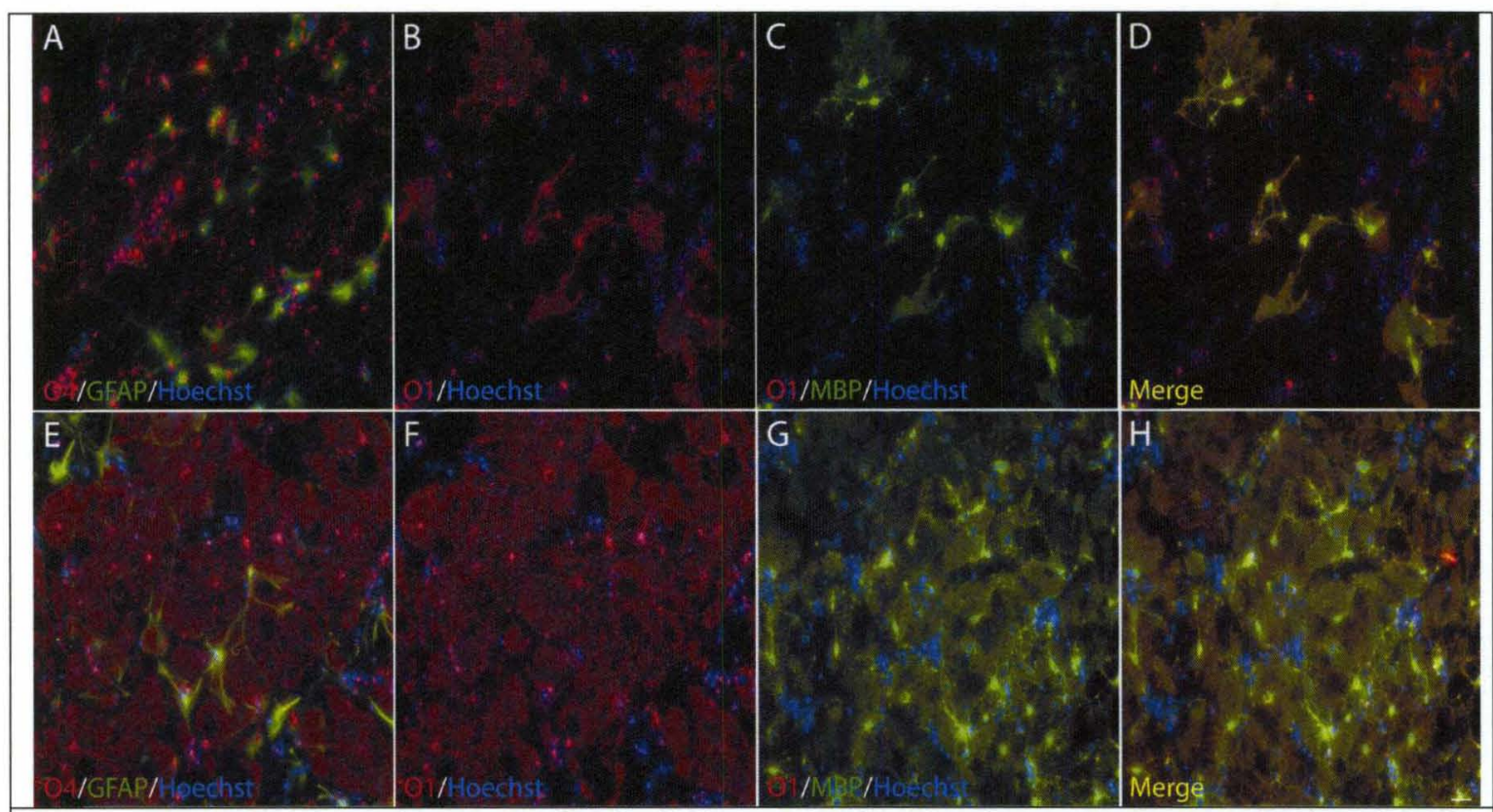

Figure 14: CNTF enhances OPC differentiation in the presence of OPC/DRG co-culture CM. (A-D) CM collected from OPC/DRG co-cultures were added to differentiating $\mathrm{P} 1$ OPCs for 7 days resulting in modest differentiation into $\mathrm{O}^{+}$and $\mathrm{MBP}^{+}$OLs. (E-H) However, when this was supplemented with CNTF, a dramatic improvement highly mature $\mathrm{O}^{+} / \mathrm{MBP}^{+}$OLs formed. This too was accompanied by $\mathrm{GFAP}^{+}$astrocyte differentiation as well. All photomicrographs are $10 \mathrm{X}$ magnification.

\section{DISCUSSION}

While numerous reports document the isolation of rat OPCs from embryonic, neonatal, and adult CNS, preparations of similarly-derived mouse OPCs has proved problematic (Lin et al., 2006; Chen et al., 2007b). Previously described mouse OPC culture techniques involve mouse embryonic neurosphere formation followed by oligosphere induction for the production of large OPC cell 
numbers (Chen et al., 2007b; Zhao et al., 2010). Immunopanning of postnatal mouse brains have previously been described. However, OPCs are positively selected on PDGFRaa or A2B5 both of which may yield reduced OPC enrichment and the latter may not select exclusively for cells that will differentiate within the oligodendroglial lineage (Larsen and Yong, 2004; Cahoy et al., 2008; Dugas et al., 2010). A2B5 ${ }^{+}$OPCs have been enriched from postnatal mouse brains by magnetic cell sorting (Larsen and Yong, 2004; Hamanoue et al., 2009). However, as the authors acknowledge, single-cell derived clones are selected for subsequent plating raising concerns that the cells are in fact immortalized and may be genomically unstable, a feature observed with cell lines. To our knowledge, this is the first $\mathrm{O}^{+} \mathrm{OPC}$ primary culture method derived from mouse postnatal cortex that generates large numbers of OPCs without relying on cell immortalization, neurospehere /oligosphere formation, or clonal expansion.

The genetic, epigenetic, and molecular identity of OPCs derived from in vitro "sphere" formation are likely to be considerably different as there induction is biologically more distant from there in vivo environment than observed with primary cultures (Gage et al., 1995; D'Amour and Gage, 2003). Furthermore, selection of cell clones derived from a single cell following isolation is likely not representative of the primary isolated OPC population (Gage et al., 1995; D'Amour and Gage, 2003). The generation of single-cell derived clones necessitates that a single OPC will undergo putative proliferative changes in order to generate subsequent clones (Lin et al., 2006). Again, these changes are 
likely not present in primary cultures, particularly when limited in passage number.

Although this method enriches OPCs based on O4 expression, once plated, they are both $\mathrm{A}_{2} \mathrm{~B}^{+}$and $\mathrm{NG}^{+}$, indicating overlapped expression of all three cell markers from cortical OPCs isolates. With P0-P1 expansion, the number of $\mathrm{O}^{+} \mathrm{OPCs}$ declines whereas $\mathrm{A} 2 \mathrm{~B} 5$ and NG2 expression is maintained. Despite reduced O4 expression, the OPCs maintain the capacity to differentiate into OLs, though the potential to become astrocytes is still present.

Consistent with reported data for both rat and mouse OPCs, these OPCs maintain the capacity to become both astrocytes and OLs (Noble et al., 1992; Nishiyama, 1998; Trotter et al., 2010). Although CNTF did reduce the extent of cell death upon differentiation (as seen with adult rat spinal cord OPCs), the number of $\mathrm{GFAP}^{+}$astrocytes increased (data not shown) (Cheng et al., 2007; Cao et al., 2010). Though not co-cultured in species matched proliferative precursors, OPC/DRG co-cultures resulted in OPC maturation into $\mathrm{O}^{+}$positive OLs with limited MBP immunoreactivity and minimal $\mathrm{GFAP}^{+}$astrocyte formation. This suggests that soluble factors in these co-cultures, such as the previously discussed NRG1, may play a relevant role in promoting preferential OPC differentiation into mature OLs rather than astrocytes (Chapter 1).

OPCs differentiated in the presence of co-culture conditioned media supplemented with CNTF resulted in extensive $\mathrm{O}^{+}$and $\mathrm{MBP}^{+} \mathrm{OL}$ formation with highly complex arborization not seen without the presence of CNTF. This suggests CNTF may be playing a synergistic role with soluble factors in the co- 
culture CM to dramatically enhance OPC differentiation. However, a considerable number of differentiated astrocytes are observed as well- a role CNTF has been previously described to be involved in (Whittemore et al., 1999).

Overall, there exists a number of limitations of coritical OPC isolation method. As the primary OPCs are passaged beyond P2, they began to morphologically resemble type 2 astrocytes. Additionally, one freeze/thaw cycle is sufficient to stress these OPCs to differentiate into astrocytes spontaneously. Therefore, fresh OPC primary cultures are necessary and it is not recommended to take them beyond P2. Despite these issues, this method permits isolation of mouse OPCs that obviate potential genomic instability and variability that occur with extended passage of other OPC culturing techniques. As a result, we contend that these OPCs are as similar as possible to their counterparts in vivo and serve as an improved in vitro model for the study of OPCs in development, disease, and trauma. 


\section{CHAPTER 3}

\section{HISTONE DEACETYLASE INHIBITION MEDIATED CYTOTOXICITY OF DEVELOPING OLIGODENDROCYTES}

\section{INTRODUCTION}

As previously discussed, post-translational protein deacetylation mediated by the 11 member mammalian superfamily of histone deacetylase complexes (HDACs) regulates chromatin remodeling, repression of gene transcription, cell cycle progression, microtubule dynamics, and cellular aging (Kazantsev and Thompson, 2008). Regulation of as histone acetylation status is tightly regulated by the activity of both histone acetyltransferases (HATs) and HDACs, of which HDACs have received attention due to their variety of function, post-translational targets, and the successful development of HDAC specific inhibitors. There are many reports of altered HDAC overexpression and activity in various human cancers relative to normal tissues (Chapter 1). This prompted the development of HDAC inhibitors. A member of the hydroxamic acid class of HDAC inhibitors, suberoylanilide hydroxamic acid (SAHA) is the first FDA approved HDAC inhibitor. It induces cell growth arrest, apoptosis, DNA damage, reactive oxygen 
species (ROS) generation, and is anti-angiogenic (Marks, 2007). In a large range of human transformed cell lines, HDACi has been implicated in causing cell death and SAHA is currently being studied in over 180 clinical trials for the treatment of a variety of malignancies, largely in combination with other chemotherapeutic agents (www.clinicaltrials.gov). This, in addition to the panHDAC selectivity of many HDAC inhibitors (including SAHA), has spurred the development of isoform selective HDACi as well, resulting in more than a dozen structurally different HDAC inhibitors currently in clinical trials (Thomas, 2009; Bertrand, 2010).

However, as toxic as HDAC inhibitors are towards transformed cells, there too is a growing body of evidence demonstrating its neuroprotective effects in a number of neurodegenerative and psychiatric disorders (Chapter 1). However, this is further complicated by multiple studies suggesting that HDAC-mediated histone deacetylation is necessary for OL differentiation (Chapter 1). These data implicate HDACs in a global repressive role in OPC differentiation into mature OLs (Shen and Casaccia-Bonnefil, 2008). Ultimately, despite putative neuroprotective effects, HDAC inhibition (HDACi) may prevent OPC maturation and lead to ineffective myelination in the developing brain.

Chemotherapy-induced cognitive impairment $(\mathrm{CICl})$ is an accepted and common adverse effect of both focal irradiation and systemic chemotherapeutic treatment in pediatric and adult patients suffering from hematological and solid malignancies (Ahles and Saykin, 2007; Janelsins et al., 2011). Clinical data show incidence of long-term $\mathrm{ClCl}$ ranging from $16 \%-75 \%$ of cancer survivors with 
either immediate to delayed onset, with the large variability attributed to cancer type, chemotherapeutic agents used, and $\mathrm{CICl}$ study methodology (Argyriou et al., 2010). Multiple mechanisms to describe $\mathrm{CICl}$ development have been suggested such as genetic predisposition, hormonal changes, DNA damage secondary to increased oxidative stress, and increased levels of inflammatory cytokines (Ahles and Saykin, 2007; Argyriou et al., 2010). Furthermore, loss of CNS progenitors and OLs, and myelinopathy secondary to clinically used chemotherapeutic agents in adult mice was demonstrated (Dietrich et al., 2006). However, despite this evidence, $\mathrm{ClCl}$ pathogenesis remains largely undefined. In this study, we investigated the role HDAC activity plays in mouse OPC survival and maturation using the blood-brain-barrier (BBB) penetrable $\mathrm{HDACi}$, SAHA. As evidenced with many transformed cell lines (Bolden et al., 2006; Lane and Chabner, 2009; Marks, 2010), treatment of normal, non-transformed primary OPCs resulted in extensive SAHA mediated toxicity. Furthermore, these in vitro results were recapitulated in vivo following SAHA treatment in postnatal day 5 mouse pups where cortical OPCs in mouse pup brains had greater induction of apoptosis than control treated animals. Though demonstrating toxicity towards transformed cell lines and even putative neuroprotection in certain models, these results demonstrate that SAHA mediated HDACi may be accompanied by the death of normal, non-transformed mouse cortical OPCs which may be catastrophic to the developing brain and spinal cord. 


\section{MATERIALS AND METHODS}

\section{Animals}

E12-E15 time pregnant C57BI/6 mice and E15 Sprague-Dawley rats were purchased from Charles River Laboratories (Wilmington, MA). CNP-EGFP mice backcrossed 6-7 generations to $\mathrm{C} 57 \mathrm{BI} / 6$ background were a generous gift from Dr. Vittorio Gallo and were bred at the University of Louisville for all in vivo experiments (Yuan et al., 2002). All animal procedures were performed according to the guidelines of University of Louisville Institutional Animal Care and Use Committee protocols and the National Institutes of Health.

\section{Mouse Cortical OPC culture}

OPCs derived from postnatal day 5-7 mouse cortices were isolated as described previously (Chapter 2 ). They were expanded to $\sim 80 \%$ confluence in 7 9 days with daily feedings of pre-warmed OPC-A media (one day following isolation, full media change; every subsequent day until passage, half media change). PO OPCs were passaged using Accutase ${ }^{\circledR}$ (Sigma, St. Louis, MO) according to the manufacturer's protocol and plated in poly-D-lysine (PDL)/laminin coated tissue coated dishes appropriate for subsequent experiments at a seeding density of $10,000-15,000 \mathrm{OPCs} / \mathrm{cm}^{2}$. Where appropriate, following five days of expansion in OPC-A media, P1 OPCs were permitted to differentiate into mature OLs for seven days with removal of PDGFAA and FGF-2 and the addition of T3 at $40 \mathrm{ng} / \mathrm{ml}$ or astrocytes in DMEM-F12 media (50\% v/v) containing $10 \%$ BSA.

RNA Extraction and reverse transcriptase PCR 
OPCs, enriched astrocytes, and OLs were lysed in TRIZOL ${ }^{\circledR}$ Reagent (Invitrogen, Carlsbad, CA) and extracted according to the manufacturer's protocol. RNA was further purified using the Qiagen MinElute Kit (Qiagen, Valencia, CA) and concentrations were confirmed by UV spectroscopy. Following total RNA isolation, $250 \mathrm{ng}$ of total RNA was used for first strand cDNA synthesis according to the manufacturer's protocol using the $\mathrm{RT}^{2} \mathrm{PCR}$ First Strand Synthesis Kit (SABiosciences Corp., Frederick, MD). As controls, samples containing all components except reverse transcriptase or no sample were treated similarly.

\section{qRT-PCR Analyses}

qRT-PCR was performed using ABI 7900HT Real-time PCR instrument (Applied Biosystems, Foster City, CA). Briefly, cDNAs were added to predesigned $\mathrm{RT}^{2} \mathrm{qPCR}$ Primer Assays (SABiosciences Corp.) for each HDAC isoform and mixed with SYBR Green/Rox Master Mix in separate reaction mixtures for all reactions and run in triplicate. The RNA levels were quantified using the $\Delta \Delta C T$ method. Expression values obtained from triplicate runs of each cDNA sample were normalized to triplicate value for GAPDH (reference gene) from the same cDNA preparation.

\section{SAHA Treatment and MTT Analysis}

P1 OPCs were plated into 96-well plates and allowed to expand for five days prior to treatment. OPC survival was assayed by measuring the conversion of the yellow, water-soluble tetrazolium, MTT (3-(4,5-dimethylthiazol-2-yl)-2,5diphenyltetrazolium bromide) to the blue, water-insoluble formazan. This 
conversion is catalyzed by cellular mitochondrial dehydrogenases. As the rate of this reaction is proportional to the number of surviving cells, the MTT assay is widely used to quantify viable cells (Ohri et al., 2011). Data are presented as the percentage of survival relative to vehicle-treated controls. SAHA (Cayman Chemical, Ann Arbor, Ml) stock was prepared in DMSO (75 mM) and diluted in appropriate media (OPC-A, astrocyte differentiation, or OL differentiation) to final treatment concentrations of $250 \mathrm{nM}, 500 \mathrm{nM}, 1 \mu \mathrm{M}$, and $2.5 \mu \mathrm{M}$. Vehicle-treated controls had DMSO concentration equivalent to the highest SAHA concentration used $(0.0033 \%)$.

\section{Total Protein and Histone Extraction for Western Analyses}

OPCs, OPC-derived enriched astrocytes, or OPC-derived OLs were washed once in DPBS and then lysed in SDS-PAGE sample buffer for cleaved caspase 3 and $\beta$-actin isolation. Histones were purified from cells or cortical tissue using the Epiquik® Total Histone Extraction Kit (Epigentek, Farmingdale, NY) according to the manufacturer's protocol. Following transfer onto nitrocellulose membranes, Western blots were blocked in 5\% milk in TBS with $0.1 \%$ Tween (TBST) for one hour at room temperature. Primary antibodies were incubated overnight in $4^{\circ} \mathrm{C}$ TBST with $5 \%$ BSA followed by secondary antibodies in TBST with $5 \%$ BSA for one hour at room temperature. Following film development, blots were digitized and quantified using the ImageQuant TL v2003.02 software. Primary antibodies used were rabbit-anti cleaved caspase 3 (1:1000 Cell Signaling, Beverly, MA), mouse-anti $\beta$-actin (1:2000), rabbit antiacetylated histone $\mathrm{H} 3$ (1:1000, Millipore, Billerica, MA), rabbit anti-acetylated 
histone $\mathrm{H} 4$ (1:1000, Abd Serotec, Raleigh, NC), mouse anti-total histone H3

(1:1000, Millipore), and mouse anti-total histone H4 (1:1000, Cell Signaling).

Secondary antibodies used were horseradish peroxidase (HRP) anti-rabbit and HRP anti-mouse $(1: 10,000)$.

\section{In Vivo SAHA Cytotoxicity and Immunohistochemistry}

Postnatal day 5 CNP-EGFP mouse pups weighing approximately $3 \mathrm{~g}$ were treated with SAHA (50 mg/kg s.c.) or vehicle ( $75 \% / 25 \% \mathrm{v} / \mathrm{v} \mathrm{DMSO/normal} \mathrm{saline,}$ s.c.) twice daily for 2.5 days. Following anesthetizing on ice, pups were transcardially perfused initially with PBS followed by $4 \%$ paraformaldehyde. Brains were then dissected and post-fixed for 48 hours followed by $30 \%$ sucrose immersion. Afterwards, brains were placed in TBS freezing media overnight and sectioned into $20 \mu \mathrm{m}$ slices using a cryostat and placed on slides.

For immunohistochemistry, sections were thawed and washed in PBS for 10 min. Then sections were blocked in 10\% normal donkey serum (NDS) and $1 \%$ BSA in PBS/0.3\% Triton (PBST) for 1 hour at room temperature. Sections were then washed in PBS for 10 min. and incubated overnight in 5\% NDS, 0.5\% BSA, and primary antibodies in PBST. The next day, sections were washed $4 \times 15 \mathrm{~min}$. in PBS and incubated in 5\% NDS, 0.5\% BSA, and secondary antibodies in PBST for 1 hour at room temperature. Sections were washed $4 \times 15 \mathrm{~min}$. in PBS mounted and cover-slipped using Flouromount G mounting media (Southern Biotech, Birgmingham, AL). Primary antibodies used included rabbit anti-cleaved caspase 3 (1:200, Cell Signaling) to visualize cells undergoing caspasedependent apoptosis and Hoechst to stain cellular nuclei. Secondary antibodies 
used were donkey anti- rabbit TRITC and donkey anti- mouse TRITC (1:200, Jackson ImmunoResearch, West Grove, PA). Sections were captured with a Nikon TiE 300 inverted microscope equipped with a DXM-1200C coded digital camera and NIS Elements software (Nikon, Melville, NY).

To assess in vivo SAHA cytotoxicity, absolute cell counts staining positive for cleaved caspase 3 and/or CNPase-EGFP were completed from 3 randomly selected sections for each animal encompassing a region of the brain that correspond to Intraaural $1.68 \mathrm{~mm}$ to $2.16 \mathrm{~mm}$ and Bregma $-2.12 \mathrm{~mm}$ to $-1.64 \mathrm{~mm}$ in the adult C57BL/ 6 brain. These were quantified by an individual blinded to animal treatments. Counts were only from cells lying within the cortex. Brain regions such as basal ganglia, brain stem, hippocampus, etc. were excluded from the analysis.

\section{Statistical Analysis}

All quantitative data are presented as means +/- S.D. qRT-PCR data analyses were completed as described above and according to the manufacturer's recommended statistical analysis methods. Statistical significance for MTT analyses as determined by conducting a One-Way ANOVA for each SAHA treatment group relative to the DMSO vehicle treated groups. Counts were averaged for each animal and an F-test to determine sample variance was completed to assess equal variance between the two treatment groups. Afterwards, statistical significance was determined using an independent t-test assuming equal variances. In vivo CNPase ${ }^{+}$and/or cleaved caspase $3^{+}$cell 
counts were analyzed by an F-test to determine sample variance after which an independent t-test assuming equal variances was conducted.

\section{RESULTS}

\section{HDAC expression characterization in mouse OPC cultures}

We characterized HDAC expression in mouse OPCs as well as OPCderived astrocytes and mature OLs and determined if any expression changes corresponded to change in proliferative state and differentiated cell type (Fig. 15). OPCs, as well as OPC-derived OLs and astrocytes expressed all HDAC isoforms (Fig. 15). More specifically, OLs showed a significant decrease in the class I HDAC2 isoform and II HDACs 4, 5, and 6 relative to proliferative OPCs maintained in growth media (Fig. 15A-C). Class I HDACs 1, 2, and 3 significantly decrease in expression during OPC differentiation into astrocytes relative to OPCs (Fig. 15A). Expression of Class lla HDAC isoforms in astrocytes relative to OPCs remain largely unchanged (Fig. 15B). Class Ilb HDAC isoform HDAC10 also significantly decreases in expression in OPC-derived astrocytes relative Undifferentiating OPCs (Fig 15C). Overall, including a variety of changes in HDAC expression that is largely decreased in OLs and astrocytes derived from OPCs relative to the undifferentiated OPCs in proliferative cell culture media, these data suggest that all HDAC isoforms have expression patterns that are dynamically regulated in each cell type. 


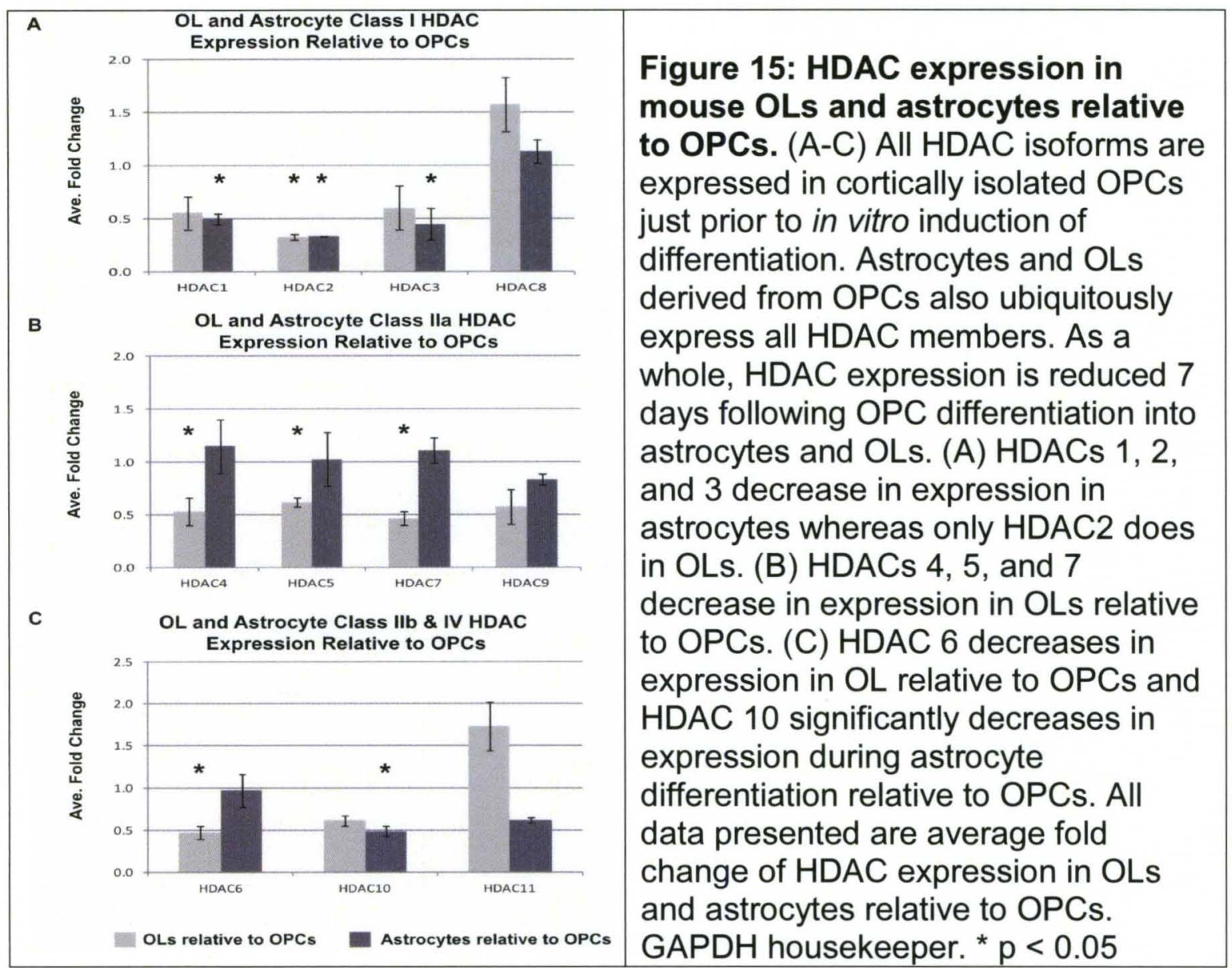

\section{SAHA mediated cytotoxicity in mouse OPCs}

To assess SAHA mediated pan-HDAC inhibition, we treated P1 OPCs in vitro in escalating doses of $250 \mathrm{nM}, 500 \mathrm{nM}, 1 \mu \mathrm{M}$, and $2.5 \mu \mathrm{M}$ for 24,48 , and 72 hours (Fig. 16). These SAHA concentrations were selected based on previous studies indicating both survivability in CNS cell cultures, but also toxicity in transformed cells lines- although SAHA concentrations below $1 \mu \mathrm{M}$ were rarely tested (Bolden et al., 2006; Bertrand, 2010; Marks, 2010). Three hours following SAHA treatment, OPCs at all concentrations showed increased levels of acetylated histone $\mathrm{H} 3$ and a dose-dependent increase in acetylated histone $\mathrm{H} 4$ relative to vehicle and growth media only treated OPCs (Fig. 16A). 


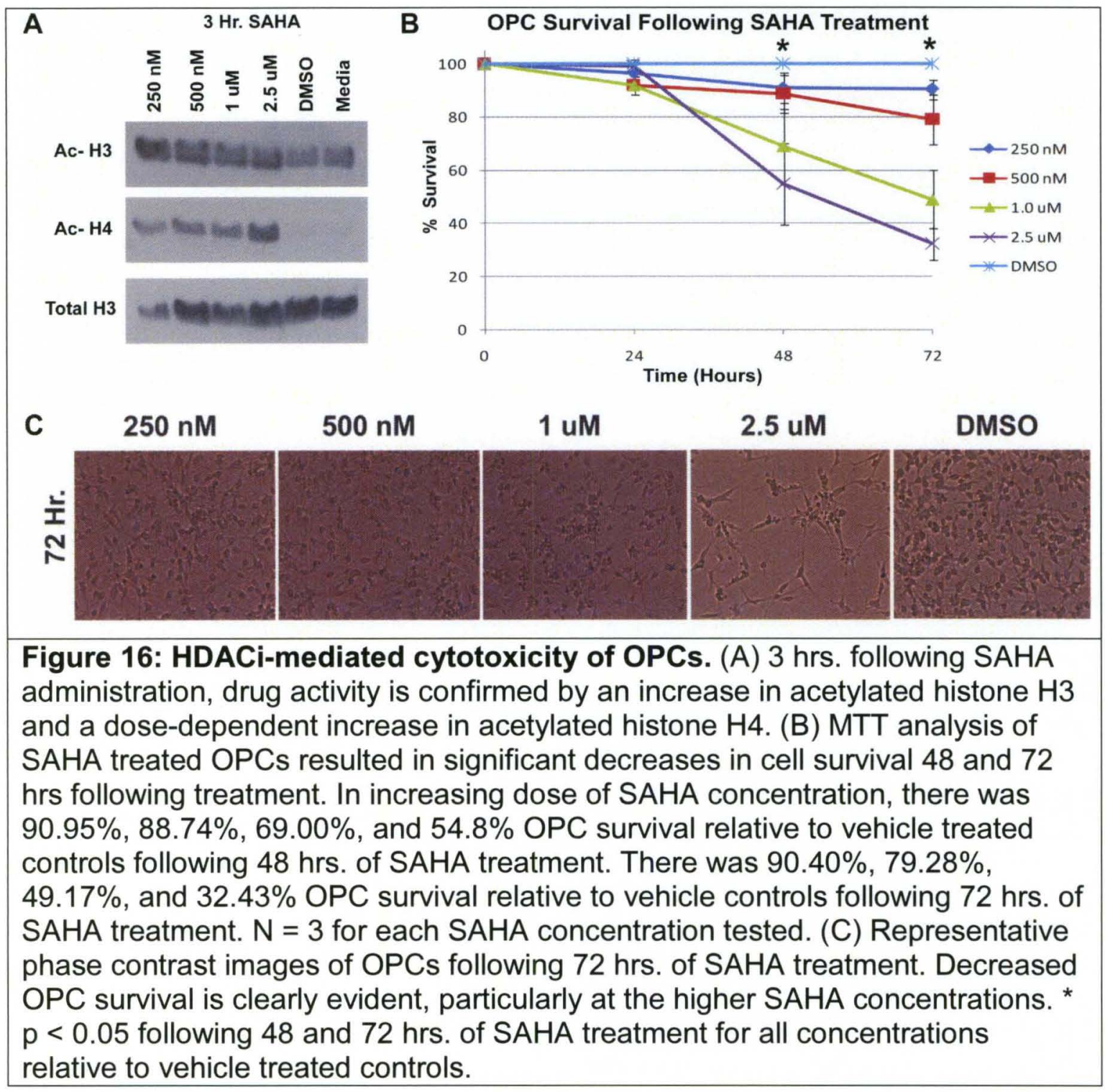

To determine the effect on OPC survival, MTT analysis revealed extensive cell death following SAHA treatment for 48-72 hours. The greatest cell death was observed at 48 and 72 hours of SAHA treatment where approximately $50 \%$ and $35 \%$ of $2.5 \mu \mathrm{M}$ treated OPCs survived relative to vehicle treated OPCs, respectively (Fig. 16B). Significant cell death was also seen at $1 \mu \mathrm{M}$ SAHA treatment where approximately half all OPCs survived relative to vehicle treated 
OPCs (Fig. 16B). Though significant, considerably less cell death was observed following 72 hours of $250 \mathrm{nM}$ and $500 \mathrm{nM}$ SAHA treatment of OPCs relative to vehicle controls (Fig. 16B). Representative images of OPCs following SAHA administration clearly illustrate the cytotoxic effect HDACi has on OPC survival at all SAHA concentrations relative to vehicle treated OPCs (Fig. 16C). The remaining cells were $\mathrm{A}_{2} \mathrm{~B}^{+}, \mathrm{O}^{+}, \mathrm{O}^{-}$, and $\mathrm{GFAP}^{-}$(unpublished observations).

To determine if differentiated OPCs have similar susceptibility to SAHA mediated cytotoxicity, OPCs were differentiated for 7 days either into astrocytes (removal of growth factors accompanied by $10 \%$ serum) or to an enriched population of complexly arborized $\mathrm{O}^{+}$and/or $\mathrm{O}^{+}$OLs. As with OPCs, the greatest cell death was seen at 72 hours of $2.5 \mu \mathrm{M}$ SAHA treatment (Fig. 17).

\begin{tabular}{|l|l|l|}
\hline & $\begin{array}{l}\text { Figure 17: OPC derived OL and } \\
\text { astrocyte survival following HDACi. } \\
\text { (A) MTT analysis revealed SAHA } \\
\text { treatment of OPC derived OLs only } \\
\text { significantly causes increased cell } \\
\text { death following } 72 \text { hrs. of treatment at } \\
1 \mu \mathrm{M} \text { and } 2.5 \mu \mathrm{M} \text { of SAHA with an } \\
\text { average of } 79.07 \% \text { and } 66.91 \% \text { cell } \\
\text { survival relative to vehicle treated } \\
\text { controls, respectively. (B) Significant } \\
\text { yet modest cell death was observed in } \\
\text { OPC derived astrocytes } 48 \text { and } 72\end{array}$ \\
& &
\end{tabular}


However, these were considerably less than observed in OPCs. OPCs following 7 days of differentiation in OL differentiation media and treated with $2.5 \mu \mathrm{M}$ SAHA for 72 hours experienced approximately a $25 \%$ loss of cell survival relative to vehicle treated controls (Fig. 17A). OPC-derived astrocytes saw a similar loss in cell survivability (Fig. 17B). Though cell death was statistically significant, it appears to be considerably more protracted and far less toxic in mature glial cells relative to their progenitors.

\section{Caspase activation following SAHA treatment in OPCs}

SAHA-mediated activation of apoptotic caspase-dependant and independent pathways in response to reactive oxygen species generation and subsequent DNA double strand breaks have been implicated in selective cell death of numerous cancer cell lines (Lee et al., 2010; Marks, 2010). As differentiated OPCs displayed a reduced susceptibility to SAHA mediated cell death relative to OPCs, we wanted to investigate whether this death was a caspase-dependant apoptosis. Cleaved caspase $3(\mathrm{CC} 3)$ is present as early as 24 hours of SAHA treatment across all four SAHA concentrations (Fig. 18A). This indicates that, at least in part, SAHA-mediated OPC death is a caspasedependent apoptotic process (Fig. 18A). We hypothesized that the greatest caspase activation likely occurs following $1 \mu \mathrm{M}$ and $2.5 \mu \mathrm{M}$ SAHA treatment. Therefore, OPCs were treated with SAHA and the potent general caspase inhibitor Q-VD-OPH following SAHA administration for 48 and 72 hours. Q-VD$\mathrm{OPH}$ administration before or at the time of SAHA administration did not improve cell survival and therefore it was determined Q-VD-OPH administration just prior 
to caspase activation (16-18 hrs. following SAHA administration) is necessary and would maximize the caspase inhibitory effect (data not shown). Phase contrast images of OPCs that received Q-VD-OPH following $2.5 \mu \mathrm{M}$ SAHA addition displayed increased cell survival (Fig. 18B). $10 \mu \mathrm{M}$ Q-VD-OPH treatment following $1 \mu \mathrm{M}$ and $2.5 \mu \mathrm{M}$ SAHA treatment resulted in nearly $50 \%$ increased cell survival relative to SAHA only treated OPCs (Fig. 18C). However, cell survival did not return to normal suggesting caspase-independent cell death pathways may be activated as well.

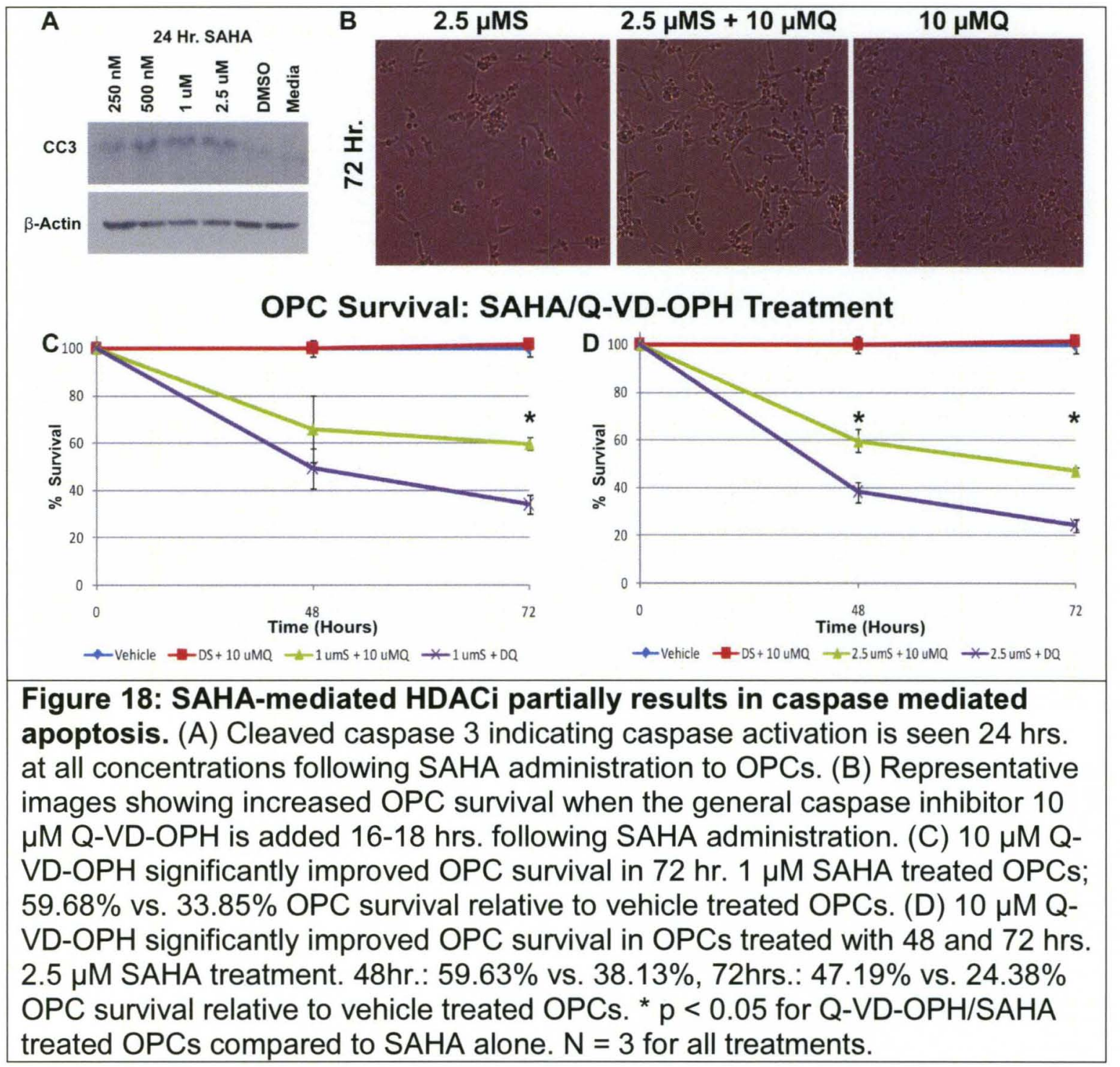




\section{SAHA is cytotoxic to cortical OPCs in vivo}

To determine whether this effect is evident in vivo as well, P5 CNPaseEGFP pups were administered either SAHA ( $50 \mathrm{mg} / \mathrm{kg}$, twice daily, s.c.) or vehicle for 2.5 days (total of 5 infusions). Though often used as a marker of oligodendroglial maturity, CNPase expression early in the oligodendroglial lineage (Yuan et al., 2002; Lytle et al., 2009). Furthermore, mouse pup brains and spinal cords begin actively myelintating between postnatal days 7-21 (Miller and Mi, 2007; Nishiyama et al., 2009). These pups were treated prior to onset of active myelination, and thus had a reduced number of mature myelinating OLs relative to adults as OPCs are only beginning to mature into OLs at this developmental time point (Wolswijk and Noble, 1989; Noble et al., 1992; Miller and Mi, 2007; Nishiyama et al., 2009). Therefore, CNPase-EGFP ${ }^{+}$cells in the brain at this age range serve as a reliable marker for OPCs in vivo.

Representative brain hemisections from mouse pup brains demonstrate the approximate regions examined for subsequent quantitative analysis (Fig. 19). Though not further investigated, brains from SAHA treated mice did appear to have reduced CNPase-EGFP expression relative to brains of vehicle treated pups (data not shown). Vehicle-treated mouse pups had multiple brain regions nearly without any cells that were $\mathrm{CC}^{+}$, particularly $\mathrm{CC}^{+} / \mathrm{CNPase}-\mathrm{EGFP}^{+}$OPCs (Fig. 20A-C). This was in stark contrast to SAHA-treated mouse pups as they had multiple brain regions, such as the lateral cortex and regions not used for 
quantification including many areas in the diencephalon, with $\mathrm{CC}^{+} / \mathrm{CNPase}^{-}$ EGFP ${ }^{+}$OPCs demonstrating induction of OPC apoptosis (Fig. 20D-I).

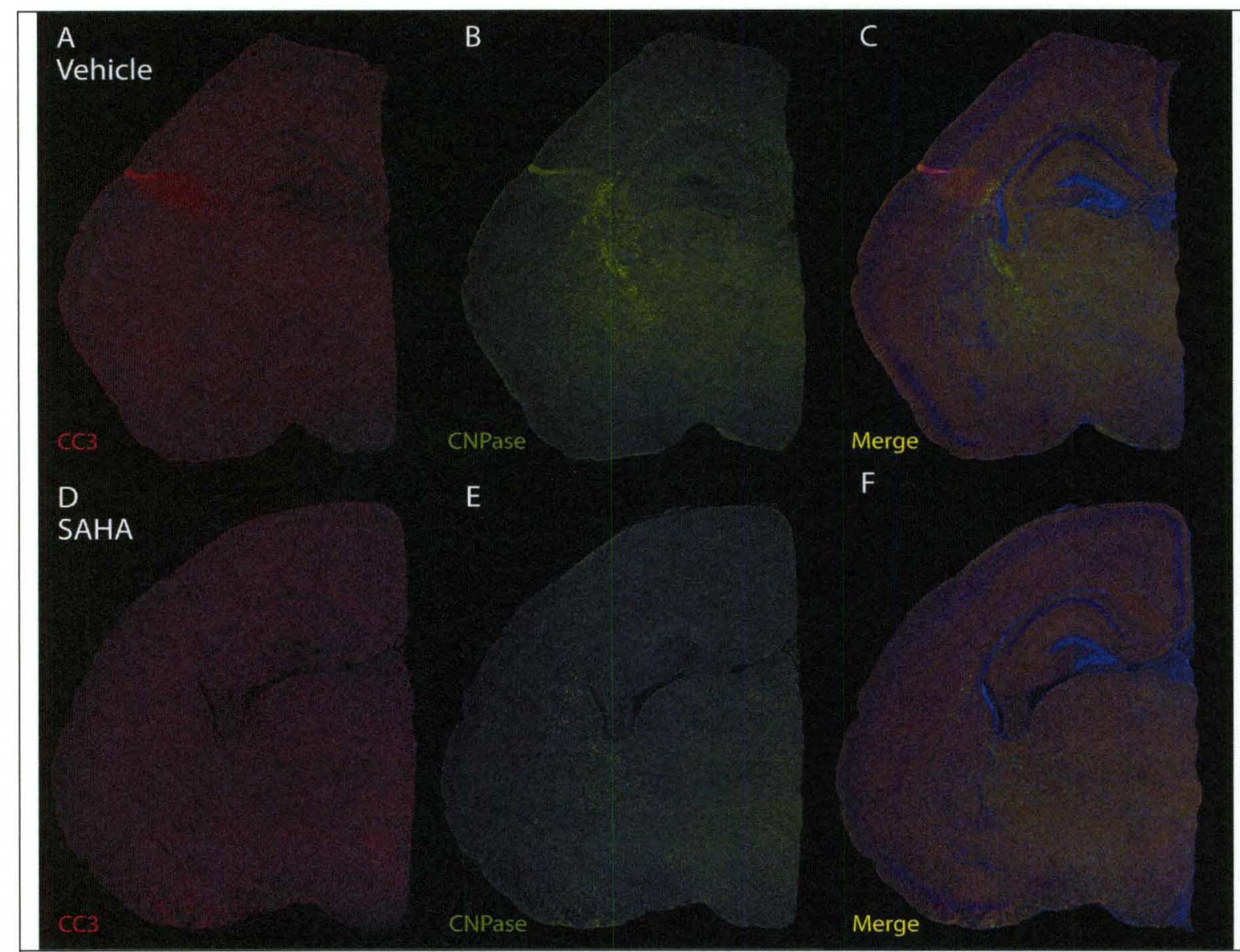

Figure 19: Vehicle vs. SAHA treated mouse brain sections. (A-F) Representative brain sections used for in vivo analysis of HDACi-mediated OPC cytotoxicity.

As our OPC cultures are derived originally from mouse cortices, we only quantified the number of $\mathrm{CC}^{+}$and $\mathrm{CC} 3 / \mathrm{CNPase}^{+}$cells from the cortex, excluding cell counts from the structures such as the brain stem, cerebellum, olfactory bulb, and hippocampus (Fig. 21A). SAHA treated animals had significantly greater $\mathrm{CC} 3$ positive cells in their cortices compared to vehicle treated mice (Fig. 21B). Additionally, SAHA treated mice had a significantly greater number of cortical $\mathrm{CC}^{+} / \mathrm{CNPase}^{+}$OPCs relative to vehicle treated animals indicating greater SAHA mediated cytotoxicity of cortical OPCs in vivo 


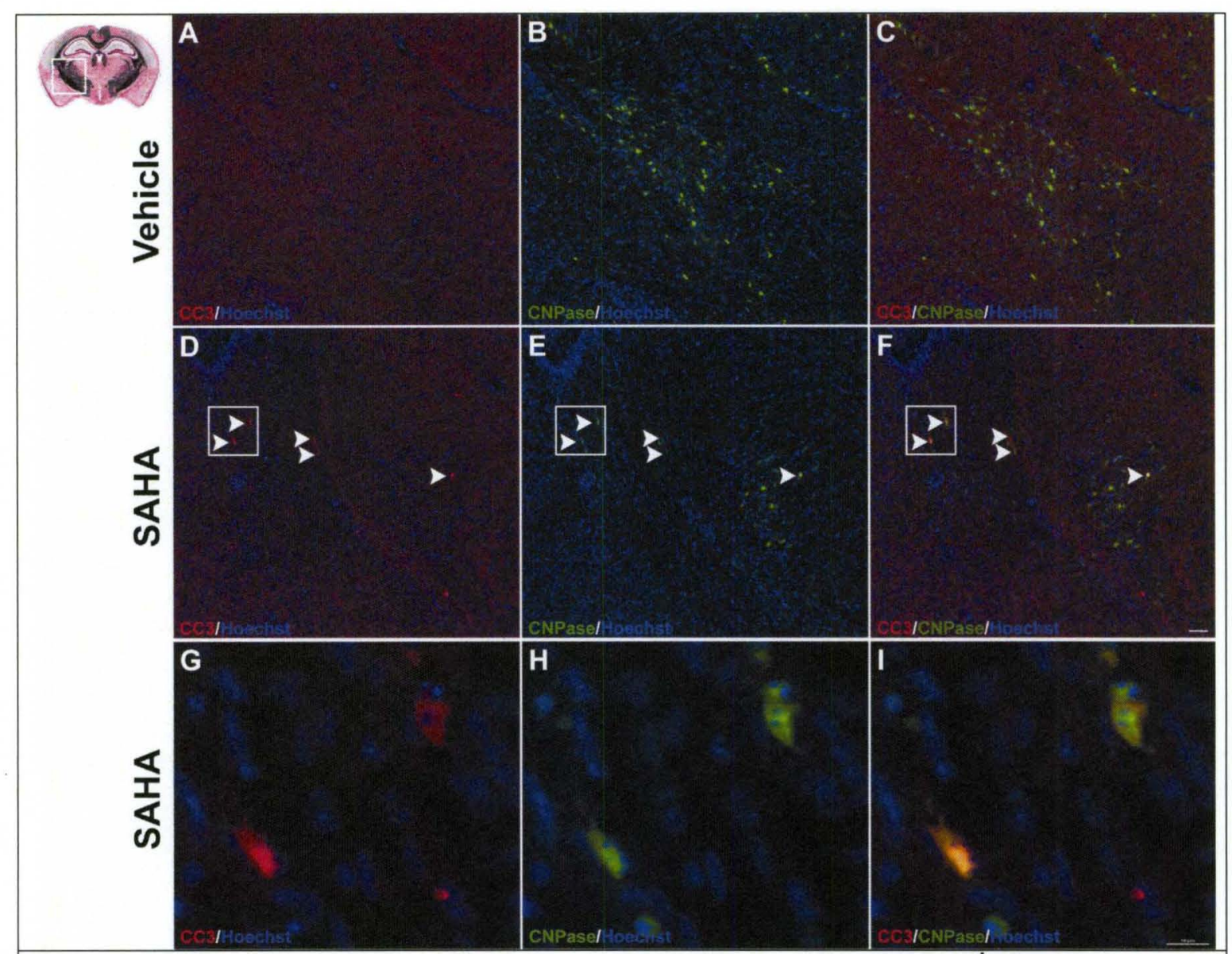

Figure 20: Co-localization of cleaved caspase 3 with CNPase ${ }^{+}$OPCs following SAHA treatment in vivo. Representative image from lateral cortex $(\mathrm{A}-\mathrm{C})$ Vehicle treated $\mathrm{P} 7$ mouse pup showing lack of CC3 immunostaining of CNPase-EGFP ${ }^{+}$OPCs and (D-F) SAHA treated P7 mouse pups showing multiple $\mathrm{CC}^{+}$immunostained cells that are also CNPase-EGFP ${ }^{+}$OPCs. (G-I) Inset of images D-F demonstrating clear CC3 immuno-staining of CNPase-EGFP ${ }^{+}$OPCs. (A-F) 10X photomicrographs, (G-I) 40X photomicrographs.

(Fig. 21C). Of interesting note, a majority of $\mathrm{CC}^{+}$and $\mathrm{CC} 3 / \mathrm{CNPase}{ }^{+}$cells are located in the lateral cortices of the developing mouse pup brain (data not shown). This may either be a byproduct of increased blood flow to those cortical regions resulting in increased SAHA exposure to effected cells or subpopulations of cells that are preferentially susceptible to HDACi-mediated cytotoxicity. 


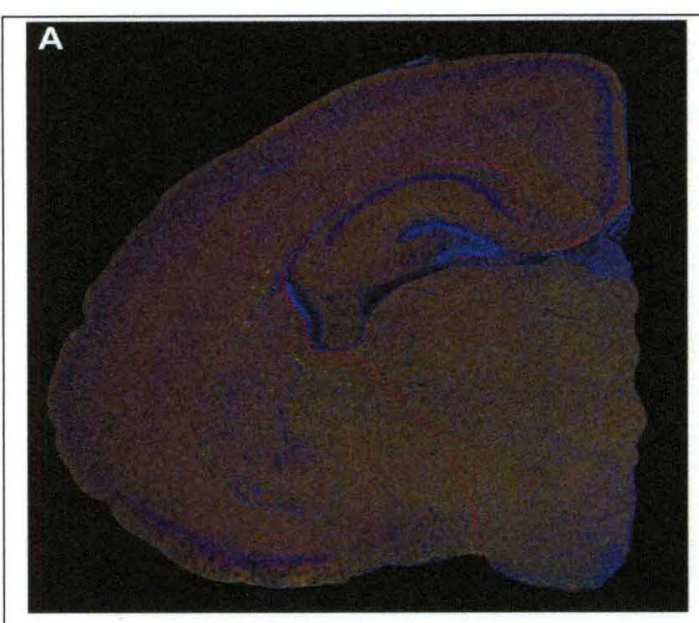

Figure 21: HDACi results in increased cortical OPC cytotoxicity in vivo. (A) Brain hemisections illustrating region excluded from cell counts. Cortical region surrounding the red line was the only area included in counts.

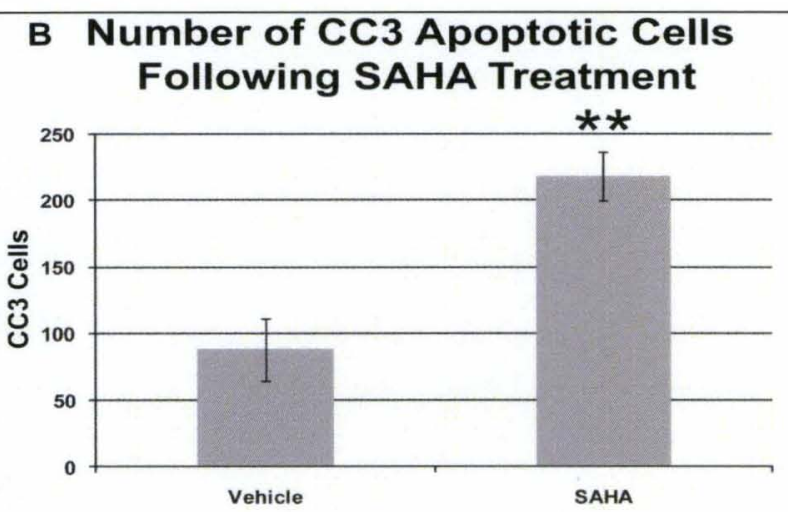

c Number of CC3/CNPase Apoptotic Cells Following SAHA Treatment

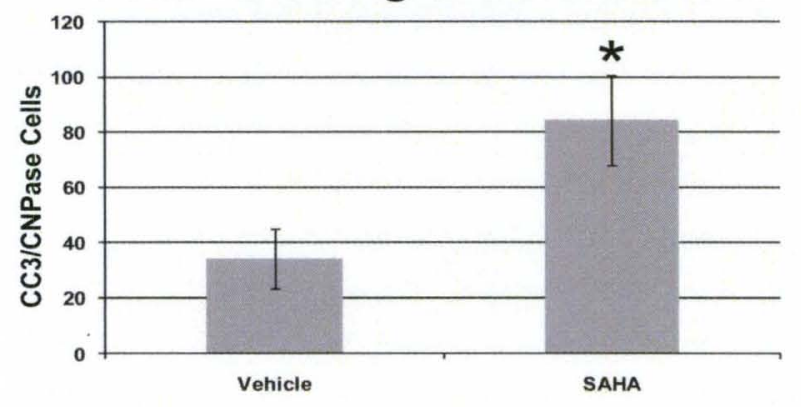

(B) There is a significant increase in the number of $\mathrm{CC}^{+}$cells/section analyzed relative to vehicle treated controls. A mean of $218.00+/-18.46$ cells were $\mathrm{CC}^{+}$ in SAHA treated brains compared to a mean of $88.11+/-22.94$ in vehicle treated animals. (C) There also is a significant increase in the number of $\mathrm{CC}^{+} / \mathrm{CNPase}-$ $\mathrm{EGFP}^{+}$cells/section analyzed relative to vehicle treated controls. A mean of $84.33+/-16.50$ cells were $\mathrm{CC}^{+}$in SAHA treated brains compared to a mean of $34.00+/-10.90$ in vehicle treated animals. ${ }^{* *} p<0.01,{ }^{*} p<0.05 . N=3$ for each group.

\section{SAHA may be cytotoxic to neurons of the developing mouse brain}

Though not quantified, there is clear evidence of specific neuronal toxicity in the superficial cortex of the mouse developing cortex in SAHA treated animals compared to vehicle treated pups (Fig. 22). SAHA treated animals have a population of $\mathrm{CC}^{+} / \mathrm{NeuN}^{+}$neurons located in superficial cortical gray matter that is absent in vehicle treated mice (Fig. 22A-D). This cell death may also account for the increased number of $\mathrm{CC}^{+} / \mathrm{CNPase}^{-}$cells that morphologically resemble neurons in SAHA treated pups suggesting the additional possibility of neuronal 
toxicity in the developing mouse brain as well as its effects on developing OLs

(Fig. 21-22).

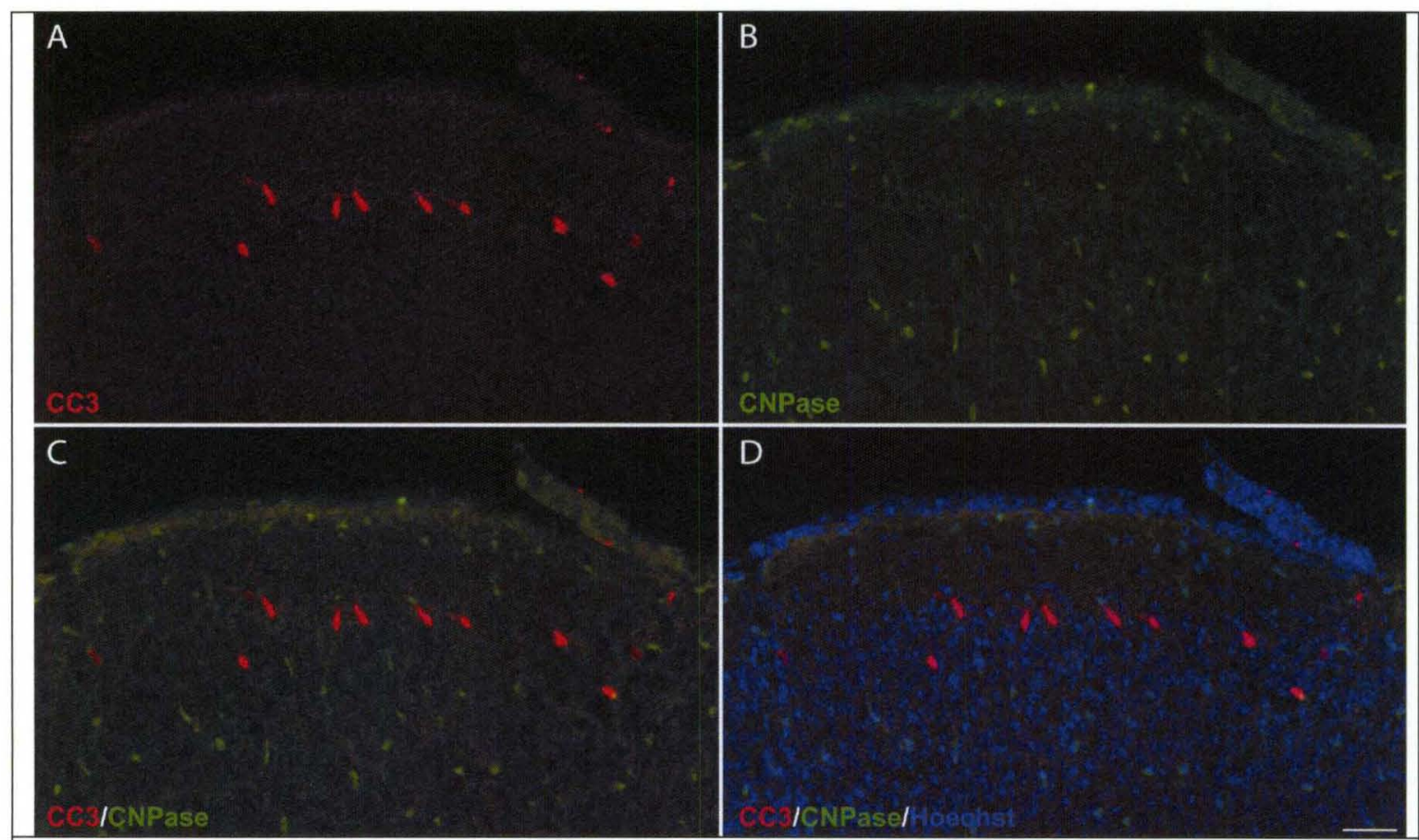

Figure 22: Putative neuronal toxicity in the superficial cortex following in vivo SAHA administration. (A) Observation of $\mathrm{CC}^{+}$cells located in the medial superficial cortex only in SAHA treated P7 mouse pups that are not (B-C) CNPase-EGFP ${ }^{+}$OPCs. Rather they morphologically resemble neurons and are and are located in a (D) cell dense region typical of cortical gray matter. Scale bar $=50 \mu \mathrm{m}$.

\section{DISCUSSION}

HDAC inhibition mediated by valproic acid and trichostatin A (TSA) promotes cell cycle arrest and increases expression of OPC maturation inhibitors, thereby preventing mature oligodendrocyte formation (Shen and Casaccia-Bonnefil, 2008; Shen et al., 2008; Pedre et al., 2011). Additionally, previous reports have indicated cytotoxicity of renal tubular cells and immature osteoblasts but studies on the developing central nervous system were lacking (Dong et al., 2008; McGee-Lawrence et al., 2011). There has been increased 
development of new HDAC inhibitors by the scientific community, putative successes of HDAC inhibitors as chemotherapeutic agents and multiple studies suggesting HDACi-mediated neuroprotective effects in vitro and in vivo (Abel and Zukin, 2008). We wanted to determine what effect transient inhibition of HDAC activity would have on OPC survival. Based on these previous data we hypothesized that SAHA-mediated HDACi may result in improved OPC cell survival in the presence of an exogenous stressor, however, we demonstrate transient SAHA-mediated HDACi leads to dramatic OPC cytotoxicity both in vitro and in vivo.

We selected a SAHA dose range that was previously shown to either be effective in reducing cell viability of transformed cells lines and/or beneficial in a neurological disease model in vitro (Bertrand, 2010; Colussi et al., 2010; Kidd and Schneider, 2010; Lee et al., 2010; Chen et al., 2011). Surprisingly, at a concentration as low as $0.5 \mu \mathrm{M}$ SAHA demonstrates cell death up to 72 hours of treatment. It was not necessary to attempt concentrations greater than $2.5 \mu \mathrm{M}$ as a large extent of the cell population had already been killed.

As OPCs are proliferative, it became necessary to determine if this cytotoxicity extended to their terminally differentiated phenotypes. The bipotentiality of this cell population made it necessary to investigate whether this toxicity extended to OPC-derived astrocytes and OPC-derived OLs separately (Fig. 17). Though a $20-30 \%$ cell death following SAHA treatment for 72 hours is observed, this effect is likely due to undifferentiated OPCs that maintained some proliferative capacity that were still susceptible to SAHA mediated cytotoxicity. 
Though astrocyte and OL death was significant at the higher SAHA concentrations relative to their vehicle treated counterparts, we sought to further determine the cause of the more robust death observed at multiple concentrations in OPCs.

Caspase activation was evident as early as 24 hours following SAHA administration and increased dramatically up to 48 hours following treatment as determined by Western analysis (data not shown). We only observed a partial increase in cell survival at the two highest tested SAHA concentrations following treatment with the general caspase inhibitor Q-VD-OPH (Fig. 18). Therefore, caspase independent pathways are also likely involved in SAHA mediated induction of apoptosis such as caspase-independent release of apoptosisinducing factor (AIF) which has been previously correlated to SAHA-mediated potentiation of transformed human leukemia and multiple myeloma cell lines when combined with doxorubicin (Cheriyath et al., 2011). Similar to other HDAC inhibitors, SAHA can induce both G1 and G2/M cell cycle arrest (Marin-Husstege et al., 2002; Bolden et al., 2006). Additional differences between MTT values may be representative of vehicle treated cells maintaining their proliferative capacity relative to SAHA treated OPCs over 72 hours of treatment. As our study examined SAHA mediated cell death, we did not direct our investigation on its effect on induction of cell cycle arrest.

As clinical trials have been initiated for administration of SAHA to pediatric patient populations suffering from a variety of hematological cancers and solid tumors, potential oligodendrotoxicity in mammalian models have yet to be 
assessed. In light of this, we were surprised to see the more than 2-fold increase in OPC death in vivo following transient SAHA administration. As SAHA treatment is typically proposed to extend over a course of $1-2$ months, the increased cell death following only 48 hours of treatment needs to be emphasized. It is important to note that although we used CC3 as our marker for in vivo cell death, this does not include OPCs that have already died, undergone caspase-independent apoptosis, or experienced extensive injury/stress but have not yet initiated caspase-dependent apoptosis. Therefore, the potential in vivo cytotoxicity is likely even greater than that measured in our study.

Though still limited in scope, a well-defined cellular and molecular basis remains elusive in $\mathrm{ClCl}$ in adults and children. A large majority of the data $\mathrm{ClCl}$ in adults comes from breast cancer survivors where decline in cognitive function is evident years following treatment. However, it still remains unclear if this decline is reversible. Cranial radiation is shown to reduce hippocampal neurogenesis in both rodent models and children undergoing focal radiotherapy for CNS tumors (Monje, 2008). Furthermore, intrathecal methotrexate for the treatment of medulloblastoma resulted in increased cognitive deficits in children (Butler et al., 1994). Therefore, chemotherapeutic agent induced CNS toxicity is dependent on the agent in question, its route of administration, ability to penetrate the bloodbrain-barrier if given peripherally, the length of treatment, and the patient's age.

We did not determine the mechanism by which SAHA-mediated HDACi causes direct OPC toxicity. It was recently demonstrated that SAHA exposure in vitro resulted in increased DNA double-strand breaks (DSBs) secondary to 
increased reactive oxygen species (ROS) generation. Furthermore, they indicated that specific transformed cell cytotoxicity by SAHA is due to their reduced capacity for DNA DSB repair compared to normal, non-transformed cells (Lee et al., 2010). However, this study used fibroblasts and, with the present evidence in mind, may call for a need to assess chemotherapeutic agent mediated toxicities on other more susceptible CNS cellular populations, such as cells of the OL lineage.

Often in assessing $\mathrm{ClCl}$, a majority of work emphasizes effects on neurotoxicity or reduction of neurogenesis. Unfortunately, work on oligodendrotoxicity following treatment with chemotherapeutic agents is limited. Carmustine, cisplatin, and cytosine arabinoside, have been demonstrated to be toxic to CNS progenitors as well as OLs (Dietrich et al., 2006). Furthermore, 5fluorouracil administration in adult rats result in delayed myelin destruction in CNS tracts (Han et al., 2008). Ultimately, additional work is necessary to determine if cognitive decline observed in $\mathrm{ClCl}$ is, at least in part, secondary to reduced OPC and/or OL survival, leading to reduced or delayed CNS myelination.

Although recent evidence suggests a putative protective role for HDAC inhibition on white matter sparing, the body of evidence implicates HDACi to be detrimental to by induction of OPC cell cycle arrest and prevention of OPC maturation (Shen and Casaccia-Bonnefil, 2008; Dietz and Casaccia, 2010). Our work adds to this by demonstrating that pan-HDAC inhibition, as mediated by the FDA approved small molecule HDAC inhibitor SAHA, induces OPC apoptosis in 
vitro and in vivo. With current use of this drug in cancer treatment, continued development of HDACi's, discussion of small molecule HDACi use in the treatment of neuropsychiatric illnesses, and over 180 clinical trials using SAHA, it is necessary to reassess the clinical use and CNS toxicity associated with $\mathrm{HDACi}$, particularly as it relates to the susceptible oligodendrocyte lineage of cells. 


\section{CHAPTER 4}

\section{SPINAL CORD-DERIVED RAT OLIGODENDROCYTE SUSUCEPTIBILITY TO HISTONE DEACETYLASE INHIBITION}

\section{INTRODUCTION}

Myelination of the central nervous system (CNS) is mediated by mature oligodendrocytes (OLs). During mammalian development oligodendrocyte precursor cells (OPCs) differentiate into OLs with subsequent CNS myelination occurring in rodents 1-3 weeks postnatally (Knobler et al., 1974; Wolswijk and Noble, 1989; Noble et al., 1992; Nishiyama, 1998). OPCs are also present in the adult mammalian CNS and may be involved in myelin turnover and remyelination in normal or diseased/injured CNS states, respectively (Thuret et al., 2006; McTigue and Tripathi, 2008). Therefore, it is important to identify mechanisms that govern OPC and OL survival and death to better understand putative disease processes that involve oligodendrocyte pathology such as multiple sclerosis, traumatic CNS injury, and leukodystrophies.

Post-translational protein deacetylation mediated by the 11 member mammalian superfamily of histone deacetylase complexes (HDACs) regulates chromatin remodeling, repression of gene transcription, cell cycle progression, 
microtubule dynamics, and cellular aging (Kazantsev and Thompson, 2008). Several studies indicate that HDAC-mediated histone deacetylation is necessary for OL differentiation (Marin-Husstege et al., 2002; Shen et al., 2008). Valproic acid (VPA)-mediated HDAC inhibition (HDACi) in neonatal rats resulted in hypomyelination of the corpus callosum due to reduction of OPC differentiation into OLs (Shen et al., 2005). Furthermore, HDAC1/2-mediated disruption of the Wnt signaling pathway is necessary for OL maturation (Li and Richardson, 2009; Ye et al., 2009). VPA treatment in cuprizone-exposed mice prevented remyelination of the corpus callosum due to reduced OPC differentiation (Shen et al., 2008). Recently, studies showing increased histone acetylation in chronic multiple sclerosis lesions in the frontal lobe white matter suggest reduced efficiency of HDAC activity which may be responsible for reduced remyelinating efficiency (Shen et al., 2008; Pedre et al., 2011).

Despite these findings, HDAC inhibitors continue to be developed and tested in numerous clinical trials in combinatorial therapies with DNA damaging chemotherapeutic agents as there is a large body of evidence implicating their efficacy as anti-cancer agents (Marks, 2010). Additionally, the previously described effects of HDAC inhibition on OL differentiation were largely on class I HDAC isoforms rather than the FDA approved and clinically used pan-HDAC inhibitor suberoylanilide hydroxamic acid (SAHA).

This has immediate implication in the understanding of chemotherapy induced cognitive impairment $(\mathrm{ClCl})$. Loss of CNS progenitors, OLs, and myelinopathy in adult mice secondary to chemotherapeutic agents as previously 
discussed and extensively reviewed (Chapter 1 ). However, as a whole, $\mathrm{ClCl}$ pathogenesis remains largely undefined. Therefore, we investigated whether SAHA mediated susceptibility is evident in rat OPCs and putative mechanisms that may be involved. Present data show both rat OPC and OL susceptibility to pan-HDACi which suggest that current clinical treatment paradigms that involve pan-HDACi may prove as toxic to the myelinating or remyelinating CNS- as they are to transformed cells, their intended target.

\section{MATERIAL AND METHODS}

\section{Rat Adult Spinal Cord OPC culture}

Rat OPCs were isolated as previously described (Talbott et al., 2006;

Cheng et al., 2007). Briefly, dissected spinal cords from adult Fischer 344 rats were minced into $1 \mathrm{~mm}^{3}$ pieces and incubated in HBSS (Invitrogen, Carlsbad, CA) containing $0.1 \%$ papain, $0.1 \%$ neutral protease, and $0.01 \%$ DNase (all from Worthington Biochemical, Lakewood, $\mathrm{NJ}$ ) for $30 \mathrm{~min}$ at $37^{\circ} \mathrm{C}$. Tissue digestion was stopped with the addition of an equal volume of DMEM containing $20 \%$ fetal bovine serum (FBS, Invitrogen). Tissue was further dissociated by repeated trituration with fire-polished Pasteur pipettes and filtered through $70 \mu \mathrm{m}$ nylon mesh. Cells were first incubated on an anti-RAN-2 antibody-coated dish for 30 min to deplete type 1 astrocytes and meningeal cells then transferred to an O4coated dish for 45 min to select OPCs. Purified OPCs on the dish were removed with trypsin (Invitrogen) and cultured in poly-d-lysine/laminin-coated dishes with DMEM/F12 medium containing Penicillin/Streptomycin (1\%), N2 (1\%) and B27 
(2\%) supplements (all from Invitrogen), fibroblast growth factor 2 (FGF2) (20 $\mathrm{ng} / \mathrm{ml}$; Millipore, Billerica, MA), platelet-derived growth factor aa (PDGFaa) (10 $\mathrm{ng} / \mathrm{ml}$; Sigma, St. Louis, MO), insulin $(5 \mu \mathrm{g} / \mathrm{ml})$, and BSA (0.1\%). Cells were fed with half-fresh growth medium every other day. In all cases, an aliquot of cells was analyzed the next day to determine the efficiency of the immunopanning and only cell preparations with $>95 \%$ of the bound cells expressed 04 were used.

\section{OPC Immunocytochemistry}

Immunocytochemical analysis of OPC cultures were done as previously described (Talbott et al., 2006; Cheng et al., 2007). Briefly, cells were washed in DPBS and then incubated with hybridoma for 45 minutes at $4^{\circ} \mathrm{C}$. Afterwards cells were fixed in $4 \%$ paraformaldehyde at room temperature for 10 minutes. Cells were blocked and incubated overnight with antibody in $4^{\circ} \mathrm{C}$ with $10 \%$ normal donkey serum (NDS, Jackson ImmunoResearch, West Grove, PA), 0.5\% BSA (Sigma), and $0.1 \%$ Triton $\mathrm{X}-100$ in DPBS. The following day, cells were incubated in secondary antibody at room temperature for one hour in $5 \%$ NDS, $0.5 \% \mathrm{BSA}$, and $0.1 \%$ triton in DPBS. Antibodies used include mouse anti-A2B5 IgM, mouse anti-O4 IgM, and mouse anti-O1 IgM (a gift from Patrick Wood, University of Miami, 70\% v/v in DPBS), rabbit anti-NG2 (1:500, Millipore), rat antiMBP (1:100, Millipore), and rabbit anti-GFAP (1:500, Dako, Glostrup, Denmark). Cells were incubated with FITC, Texas Red, and Rhodamine Red conjugated $F\left(a b^{\prime}\right)_{2}$ fragment antibodies (Jackson Immunoresearch). Negative controls for antibody staining included the appropriate species-specific non-immune IgG or IgM antibodies. 10X and 20X photomicrographs were captured with a Nikon TiE 
300 inverted microscope equipped with a DXM-1200C coded digital camera and NIS Elements software (Nikon, Melville, NY). 63X photomicrographs were captured with the Zeiss AxioObserver inverted microscopes with AxioVision software (Carl Zeiss Microlmaging, Thornwood, NY).

\section{Drug Treatments and MTT Analysis}

P1 OPCs were plated into 96-well plates and allowed to expand for five days prior to treatment. OPC survival was assayed by measuring the conversion of the yellow, water-soluble tetrazolium, MTT (3-(4,5-dimethylthiazol-2-yl)-2,5diphenyltetrazolium bromide; Sigma) to the blue, water-insoluble formazan. This conversion is catalyzed by cellular mitochondrial dehydrogenases. As the rate of this reaction is proportional to the number of surviving cells, the MTT assay is widely used to quantify viable cells (Ohri et al., 2011). Data are presented as the percentage of survival relative to vehicle-treated controls. SAHA ( $75 \mathrm{mM}$ in

DMSO; Cayman Chemical, Ann Arbor, MI), Q-VD-OPH (20 mM in DMSO; R\&D Systems, Minneapolis, MN), and Pifithrin- $\alpha$ (50 mM in DMSO; EMD Chemicals, Rockland, MA) stocks were prepared and diluted in appropriate media (OPC or differentiation) to final treatment concentrations.

\section{Protein Extraction and Western Analyses}

Cells were washed once in DPBS and then lysed either in SDS-PAGE sample buffer or NTEN buffer for separate nuclear lysates. Following transfer onto nitrocellulose membranes, Western blots were blocked in 5\% milk in TBS with $0.1 \%$ Tween-20 (TBST) for one hour at room temperature. Primary antibodies were incubated overnight in $4^{\circ} \mathrm{C}$ TBST with $5 \%$ BSA followed by 3 
washes for 10 minutes in TBST, then addition of secondary antibodies in TBST with $5 \%$ BSA for one hour at room temperature. Primary antibodies used were rabbit anti-cleaved caspase 3 (1:1000 Cell Signaling, Beverly, MA), mouse-anti $\beta$-actin (1:2000), rabbit anti-acetylated histone H3 (1:1000, Millipore, Billerica, MA), mouse anti-total histone $\mathrm{H} 3$ (1:1000, Millipore, Billerica, MA), rabbit antihistone $\mathrm{YH} 2 \mathrm{~A} . \mathrm{X}(1: 1000$, Abcam, Cambridge, MA), and rabbit antiphosphorylated P53 (1:1000). Secondary antibodies used were horseradish peroxidase (HRP) anti-rabbit and HRP anti-mouse $(1: 10,000)$.

\section{RESULTS}

\section{SAHA mediated cytotoxicity of adult rat OPCs}

Adult rat spinal cord-derived OPCs display similar susceptibility to SAHAmediated HDAC inhibition as previously demonstrated in postnatal mouse cortical OPCs (Chapter 3). Adult rat OPC primary cultures ranging from P6-P10 are entirely $\mathrm{A}_{2} \mathrm{B5}^{+}$but largely NG2 (Fig. 23A). Furthermore, they are $\mathrm{O}^{+}$and do not have any $\mathrm{GFAP}^{+}$astrocytes, indicating the purity of this primary culture of adult rat OPCs (Fig. 23B). $\mathrm{O} 1$ and myelin basic protein (MBP) immunostaining are entirely absent demonstrating a continued proliferative capacity and a lack of spontaneous differentiation by OPCs in vitro (Fig. 23C). 3 hours after SAHA treatment, OPC cultures display an increase in acetylated histone $\mathrm{H} 3$ relative to vehicle treated OPCs indicating HDAC inhibition as acetylated histone subunits are a common HDAC substrate (Fig. 23E). Following SAHA addition, cell death is seen as early as 24 hours of treatment (Fig. 23D). Cell survival ranges 
approximately from $57 \%(2.5 \mu \mathrm{M})$ to just less than $76 \%$ (250 nM) following 24

hours of SAHA treatment. 48-72 hours of SAHA treatment results in continued

cell death with OPC survival being as low as $14 \%(2.5 \mu \mathrm{M})$ following 72 hours of treatment relative to vehicle treated OPCs (Fig. 23E). It is interesting to note that cell death nearly plateaus after 24 hours of exposure to $250 \mathrm{nM}$ SAHA as there is only a $7-8 \%$ difference in cell survival between 72 hours and 24 hours of treatment (Fig. 23E).
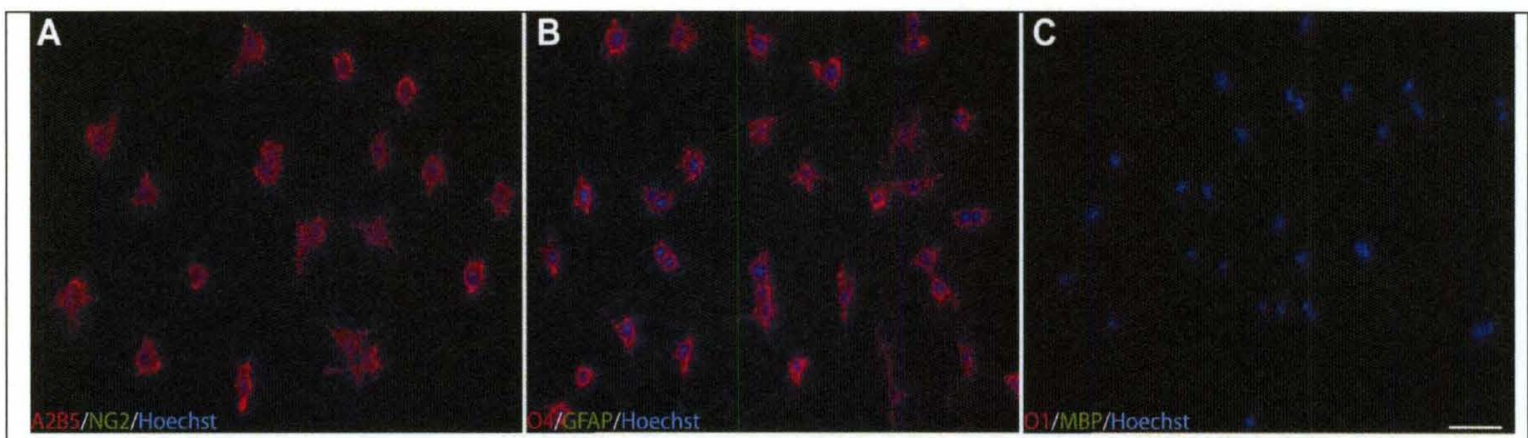

D

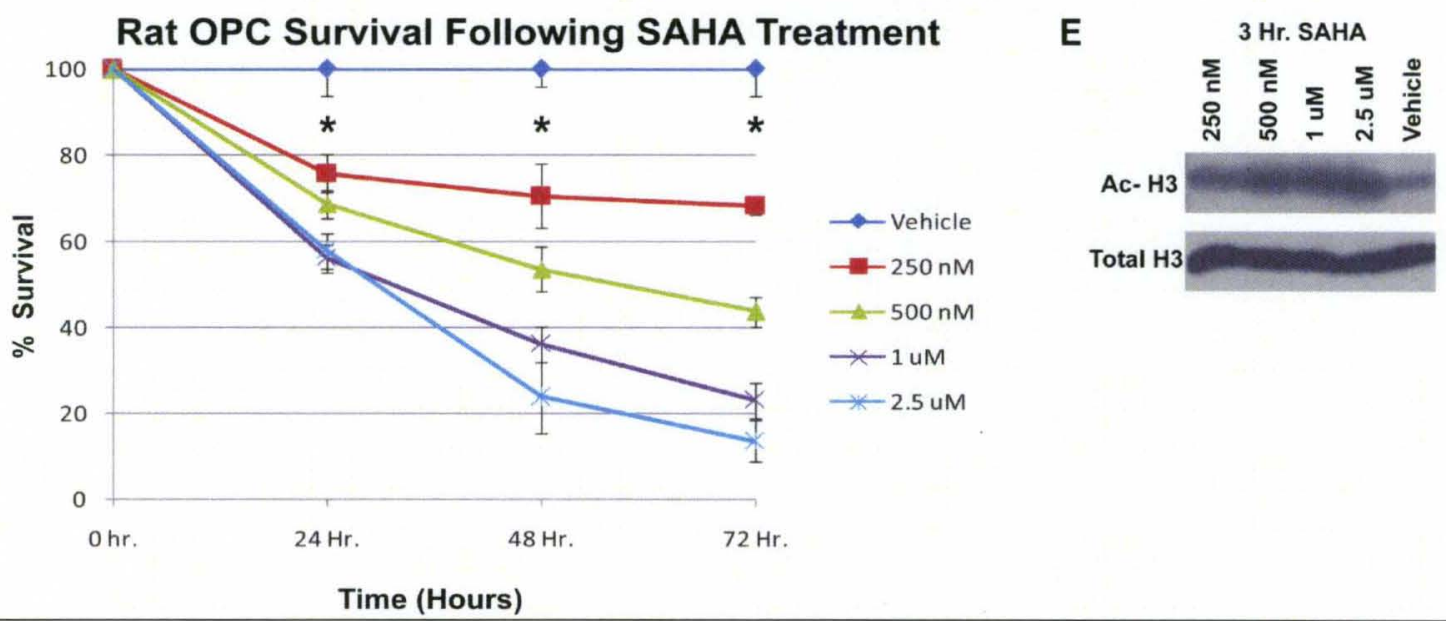

Figure 23: HDACi-mediated cytotoxicity of rat OPCs. Spinal cord- derived rat OPCs are (A) $\mathrm{A}^{2} \mathrm{~B}^{+} / \mathrm{NG}^{-}$, (B) $\mathrm{O}^{+} / \mathrm{GFAP}^{-}$and (C) $\mathrm{O}^{-} / \mathrm{MBP}^{-}$indicating a purified population of OPCs that can be maintained at least through $\mathrm{P} 10$ without phenotypic drift. (D) From 24-72 hrs. following SAHA administration, significant decreases in cell survival are observed by MTT analyses. Cell survival is at a minimum at $72 \mathrm{hr} .1 \mu \mathrm{M}$ and $2.5 \mu \mathrm{M}$ SAHA treatment where $23.20 \%$ and $13.75 \%$ of OPCs remain, respectively. (E) SAHA administration results in appropriate drug activity in vitro as evidenced by an increase in the amount of acetylated histone $\mathrm{H} 3$ across all SAHA concentrations as early as $3 \mathrm{hrs}$. following treatment. ${ }^{*} \mathrm{p}<0.05, \mathrm{n}=3$ for all treatments. 


\section{HDACi-induced DNA damage in rat OPCs}

Multiple transformed cell lines have been demonstrated to undergo DNA damage following small molecule HDAC inhibition (Bolden et al., 2006; Lane and Chabner, 2009; Bertrand, 2010). Therefore, we investigated whether this was present in rat OPCs as well. Using an antibody against histone $\mathrm{yH} 2 \mathrm{AX}$, a marker highly suggestive of DNA double strand breaks (DSBs), multiple $\mathrm{yH}^{\mathrm{A} A X^{+}}$foci are evident following rat OPC exposure to SAHA (Fig. 24). As early as 6 hours, $\mathrm{YH}_{2} \mathrm{AX}{ }^{+}$immunostaining is observed in OPC nuclei following treatment with $1 \mu \mathrm{M}$ and $2.5 \mu \mathrm{M}$ SAHA (Fig. 24A, D). By 12 hours following treatment, both SAHA drug concentrations show increased $\mathrm{pH} 2 \mathrm{AX}$ immunostaining (Fig. 24B, E). 24 hours following SAHA treatment, increased $\mathrm{YH} 2 \mathrm{AX}$ staining indicates accumulation of DNA damage in rat OPCs over the treatment period (Fig. 24C, F). 24 hour treatment of OPCs with vehicle $(0.003 \%$ DMSO) displayed minimal $\mathrm{YH}_{2} \mathrm{AX}^{+}$immunostaining relative to all SAHA-treated OPCs (Fig. 24H). Western analysis of nuclear lysates 18 hours following SAHA addition show a dose dependant accumulation of DNA damage relative to vehicle treated OPCs (Fig. 24G). This evidence strongly suggests accumulation of DNA damage, likely to be DNA DSBs, following SAHA treatment of OPCs. 


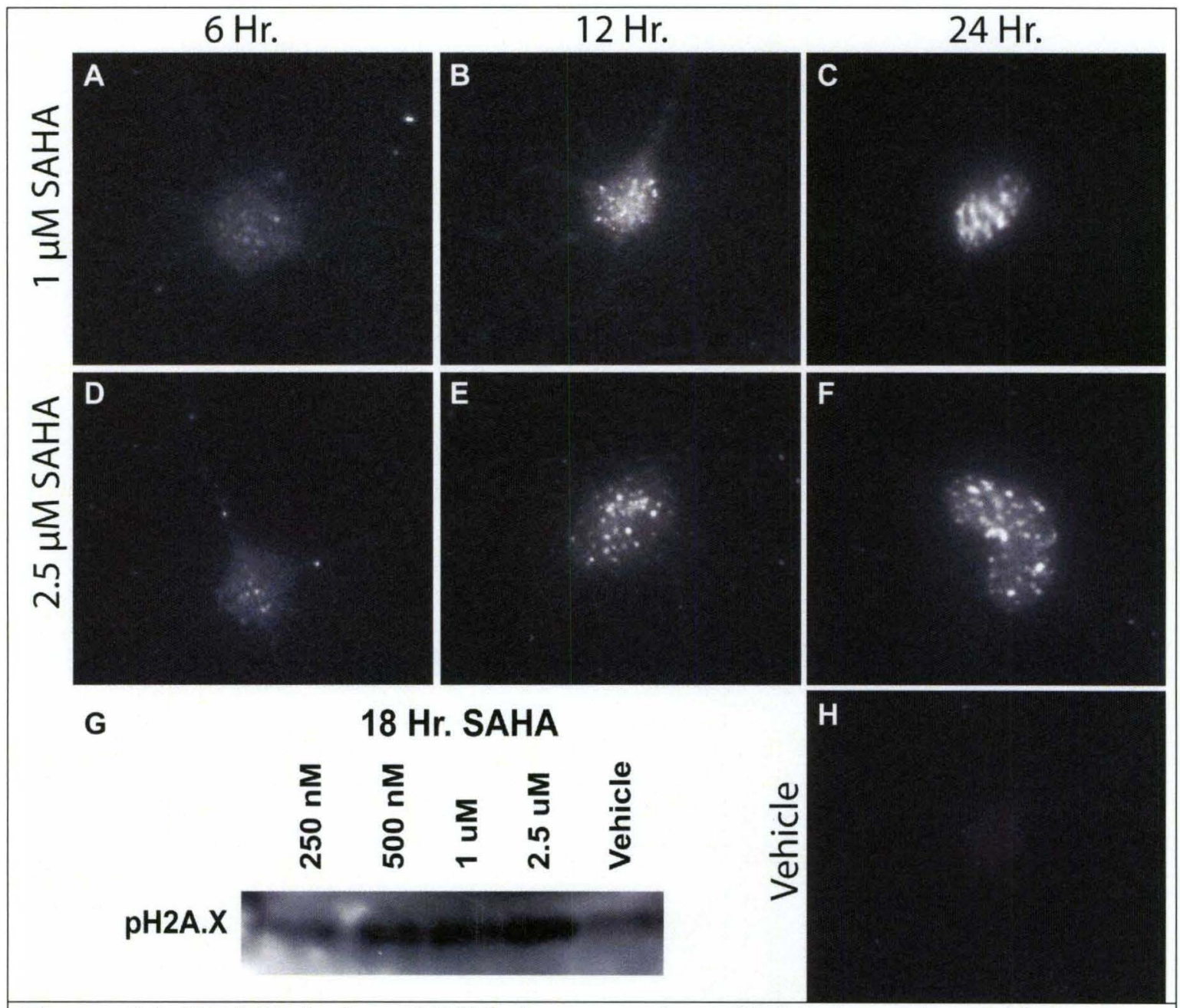

Figure 24: HDACi- induced DNA damage of rat OPCs. As early as $6 \mathrm{hrs}$. following SAHA administration, rat OPCs display evidence of DNA damage with numerous $\mathrm{yH} 2 \mathrm{AX}$ nuclear foci at (A) $1 \mu \mathrm{M}$ and (B) $2.5 \mu \mathrm{M}$ SAHA. These foci continue to accumulate in number through $(B, E) 12$ hrs. and $(C, F) 24$ hrs. following SAHA treatment. The foci are considerably greater in intensity and number compared to $(\mathrm{H})$ vehicle treated OPCs. $(\mathrm{G})$ Western blot analysis shows an increase in $\mathrm{YH} 2 \mathrm{AX}$ immunoreactivity from nuclear lysates of OPCs isolated 18 hrs. following SAHA administration relative to vehicle treated OPCs.

\section{p53 and caspase activation following HDAC inhibition}

Previously, we demonstrated HDACi-mediated apoptosis in mouse OPCs is partly a caspase-dependant process. Caspase activation is also observed following SAHA treatment in rat OPCs. A dose dependant increase in cleaved caspase 3 is seen by 24 hours of treatment with SAHA relative to vehicle treated 
controls (Fig. 25A). As early as 6 hours following SAHA addition, increased levels of phosphorylated p53 (pP53) is observed by Western blot analysis (Fig. 25B).

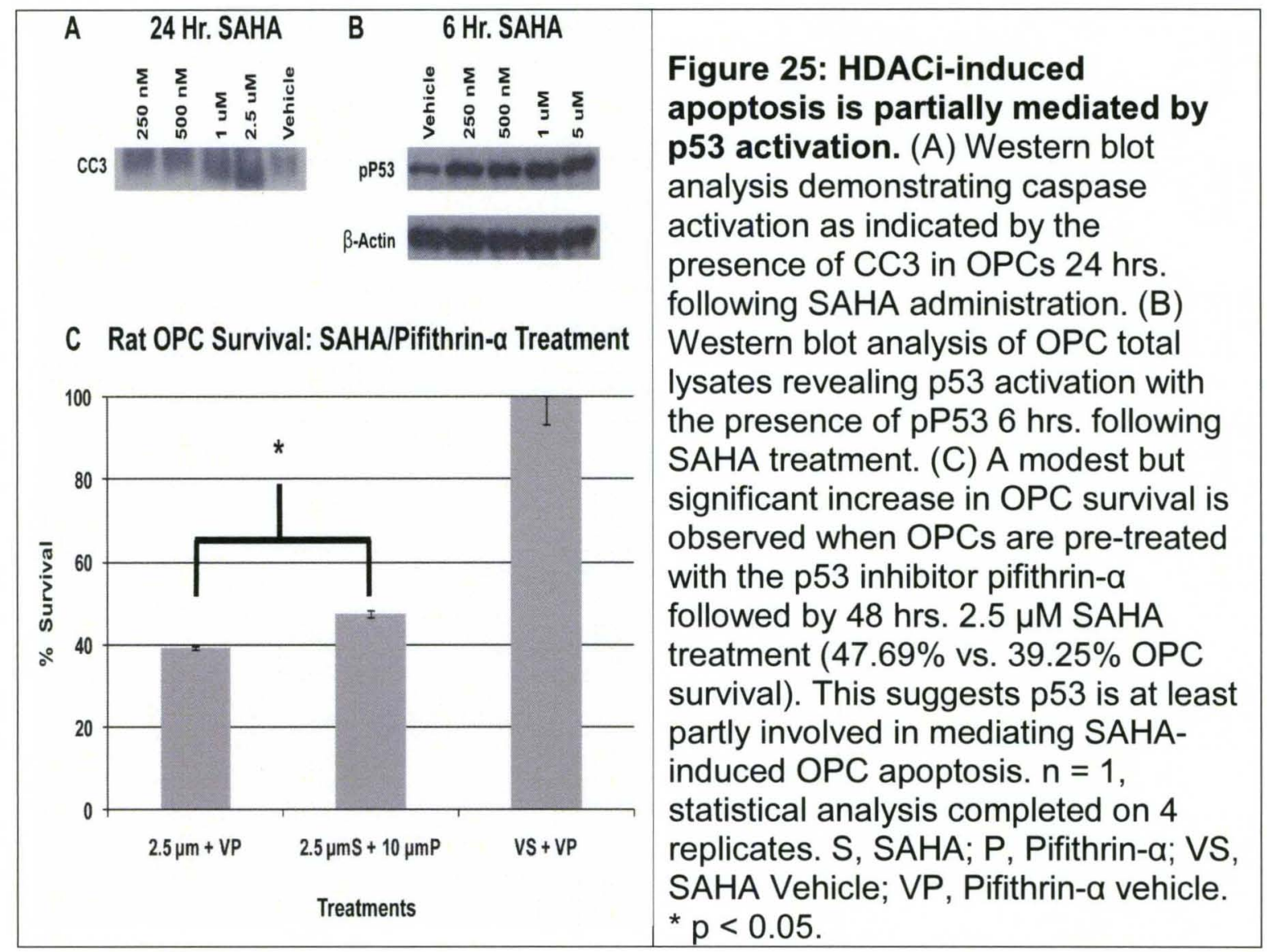

Combined, these data suggest p53 activation may be responsible for caspase activation followed by apoptotic induction. To test this, we combined SAHA treatment of rat OPCs with the well known p53 inhibitor pifithrin- $\alpha$. However, following 48 hours of $2.5 \mu \mathrm{M}$ SAHA $(2.5 \mu \mathrm{M}) /$ pifithrin- $\alpha(10 \mu \mathrm{M})$ treatment, only a modest, yet significant $8 \%$ increase in cell survival is observed (Fig. 25C). This increase in cell survival is also observed at a lower $1 \mu \mathrm{M}$ SAHA treatment (data not shown). These results suggest that although caspase mediated apoptosis in 
SAHA treated OPCs is likely secondary to p53 activation, inhibiting p53 activity is insufficient to dramatically improve cell survival.

\section{HDACi does not enhance etoposide mediated OPC death}

FDA approved and clinical trial use of HDACi against malignancies typically involves combinatorial therapy with DNA damaging agents (Bolden et al., 2006; Frew et al., 2009). Etoposide, a topoisomerase II inhibitor, is one such agent and results in DNA DSBs in proliferative transformed cells. Though no direct effects on topoisomerase II by SAHA have been previously noted, SAHA has been demonstrated to work syngertistically with topoisomerase II inhibitors by promoting chromatin decondensation and permitting increased DNA binding of the topoisomerase II inhibitor potentiating DNA damage in breast cancer cell lines (Marchion et al., 2004). However, it was recently demonstrated that etoposide, when combined with $\mathrm{HDACi}$, is cytotoxic to normal postnatal rat cortical neurons (Michal Hetman, unpublished results).

Therefore, we determined whether this was the case in rat OPCs as well. 24 hour treatment of rat OPCs with etoposide, using drug ceontrations from 0.25 $\mu \mathrm{M}$ to $2.5 \mu \mathrm{M}$, results in OPC death ranging from $32-55 \%$, respectively (Fig. 26A). By 48 hours of exposure to etoposide, nearly all rat OPCs are dead across all tested concentrations (data not shown). When $0.5 \mu \mathrm{M}$ or $1 \mu \mathrm{M}$ etoposide treatment is combined with $0.5 \mu \mathrm{M}$ or $1 \mu \mathrm{M}$ SAHA, a synergistic effect on rat OPC death is not observed (Fig. 26B-C). As etoposide induces p53-mediated, caspase-dependant apoptosis, the absence of synergism between etoposide and 
SAHA suggest these compounds may result in cell death via separate DNA damaging pathways in rat OPCs.

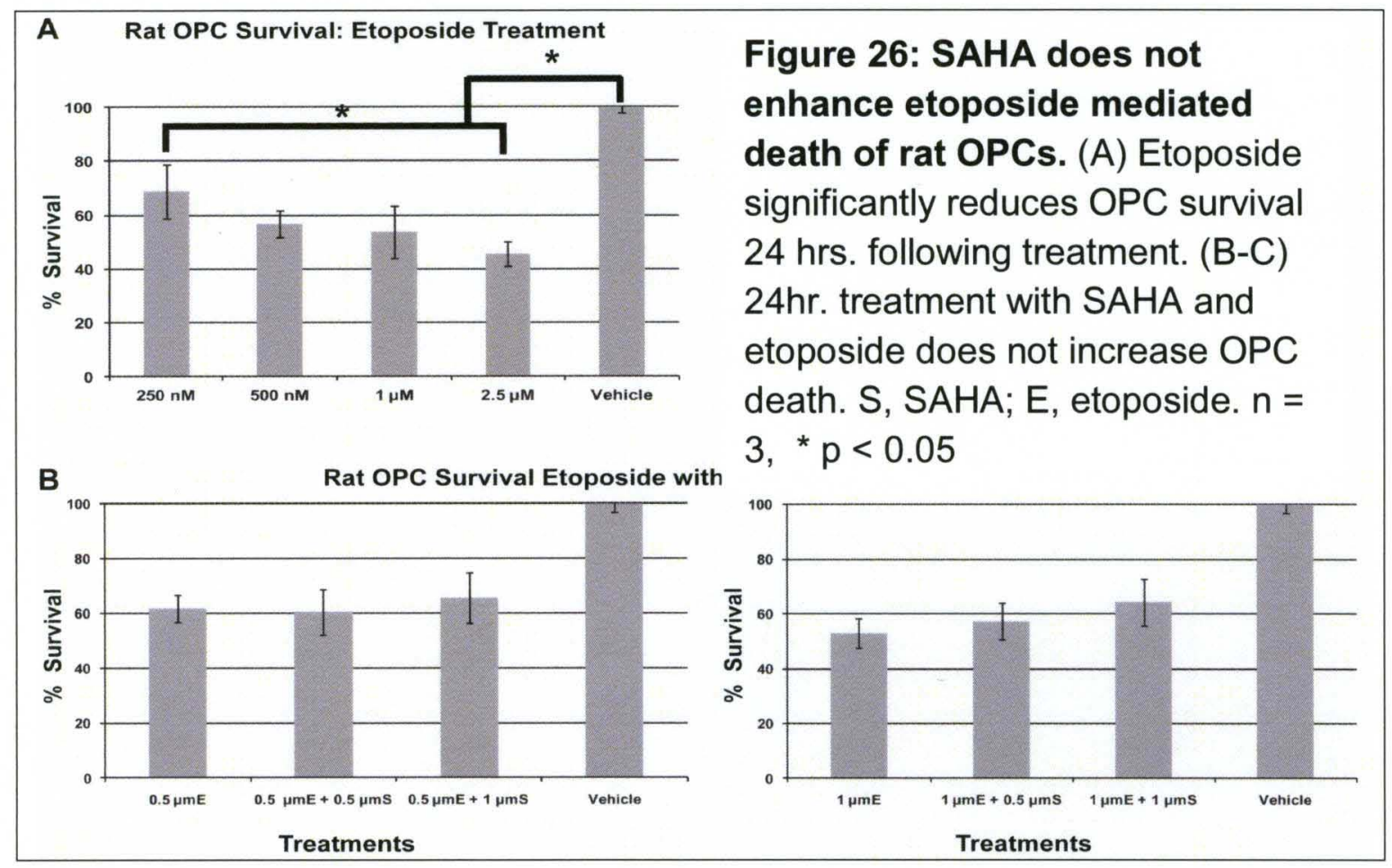

\section{HDACi mediated cytotoxicity of rat OLs}

Previously, mouse OPCs differentiated to either astrocytes or OLs in vitro resulted in a statistically significant but small reduction in cell survival following 72 hrs. SAHA treatment (Chapter 3). In rat OPC-derived OLs, withdrawal of growth factors and transfer to differentiation media for 7 days, results in differentiated OPCs with phenotypic characteristics of mature OLs (Fig. 27). Differentiated OLs maintain their A2B5-and O4-positive immunostaining, but display a more complex arborization pattern of processes than the typically biand tri-polar OPCs (Fig. 27A-B). Rat OLs are $\mathrm{O}^{+}$, a cell surface marker associated with more mature OLs (Fig. 27C). Additionally, $\mathrm{O}^{+}$OLs co-express MBP indicating full differentiation into mature OLs (Fig. 27D-E). All cells are 
GFAP', therefore it is a pure population of mature OLs (data not shown). In contrast to mature mouse OLs, rat OLs display the same susceptibility to SAHAmediated HDAC inhibition as OPCs. 24 to 72 hours of $250 \mathrm{nM}$ to $2.5 \mu \mathrm{M}$ SAHA treatment resulted in significantly reduced cell survival (Fig. 27F).

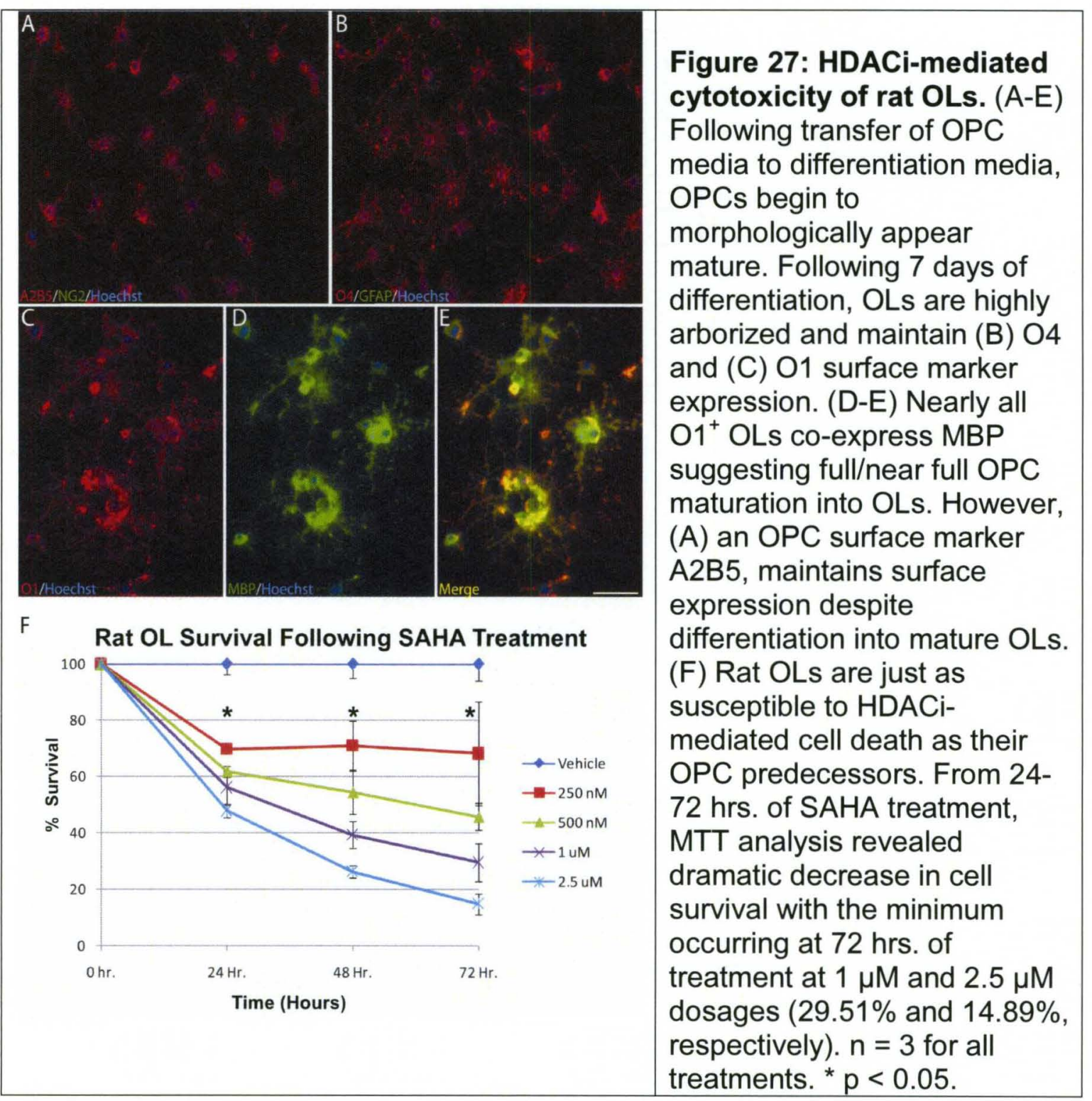

To determine whether rat $\mathrm{OL}$ death is dependent on caspase activation as in rat OPCs we treated rat OLs with $1 \mu \mathrm{M}$ or $2.5 \mu \mathrm{M}$ SAHA accompanied by the 
potent general caspase inhibitor q-vd-oph. $10 \mu \mathrm{M}$ q-vd-oph resulted in a modest increase in cell survival in 24-48 hour $1 \mu \mathrm{M}$ SAHA-treated rat OLs (Fig. 28A). An increase in rat OL survival is also seen with $2.5 \mu \mathrm{M}$ SAHA treatment for 24-48 hours (Fig. 28B). Overall, there is a $10-17 \%$ increase in rat $\mathrm{OL}$ survival when either $1 \mu \mathrm{M}$ or $2.5 \mu \mathrm{M}$ SAHA treatment is combined with $10 \mu \mathrm{M}$ q-vd-oph relative to SAHA alone suggesting that cell death is at least in part due to caspasemediated apoptosis. To establish whether this may be secondary to activated p53, $1 \mu \mathrm{M}$ and $2.5 \mu \mathrm{M}$ SAHA treatment of rat OLs was combined with pifithrin-a for 24-48 hours. At 24 hours following combined treatment, SAHA/pifithrin- $\alpha$ treated cells showed an increase of $17-27 \%$ cell survival at $2.5 \mu \mathrm{M}$ and $1 \mu \mathrm{M}$ relative to SAHA only controls, respectively (Fig. 28C-D). However, this protective effect was transient as rat OLs with the combined treatment show no difference in cell survival relative to SAHA only treated cells following 48 hours of treatment (Fig. 28C-D). These data suggest that SAHA mediated cytotoxicity of rat OLs is also in part a p53 activation mediated caspase-dependent apoptosis. 


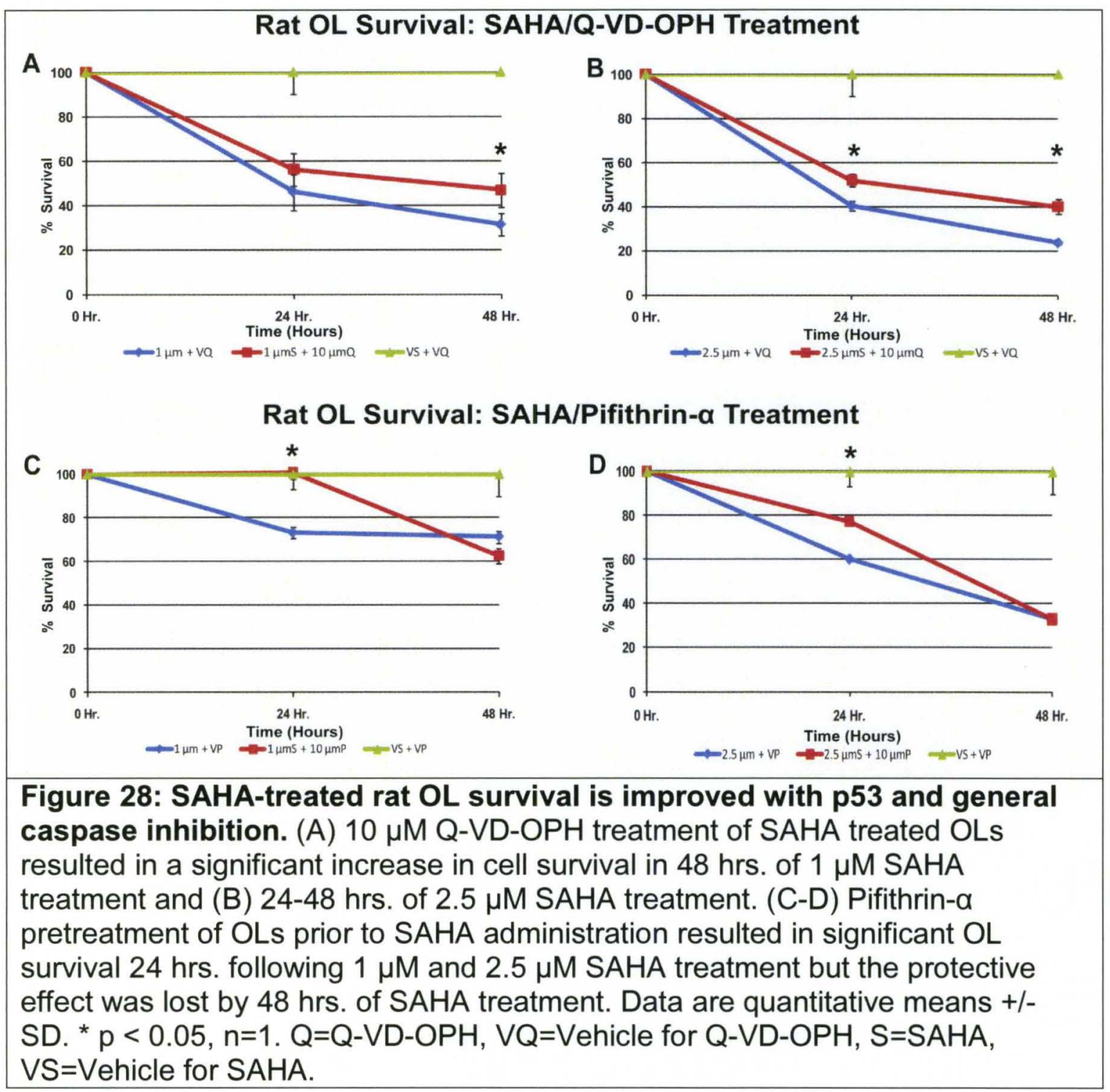

\section{DISCUSSION}

Clinical use of laboratory and commercially developed HDAC inhibitors is likely to expand quickly given their considerable anti-neoplastic effects.

Furthermore, with recent evidence suggesting improvement of survival in various models of neuronal death, use may include non-cancerous conditions as well (Abel and Zukin, 2008; Thomas, 2009). Although previously HDACi has been shown to result in OPC cell cycle arrest, direct HDACi mediated rat OPC 
apoptosis had yet to be demonstrated (Marin-Husstege et al., 2002; Shen and Casaccia-Bonnefil, 2008; Shen et al., 2008). Here we show the FDA approved specific pan-HDAC inhibitor, SAHA, causes DNA damage, results in p53 activation, and induces caspase-dependent apoptosis. Lastly, we demonstrate that pan-HDACi susceptibility is also present in rat OLs, which is in slight contrast to the modest cytotoxicity present in HDAC inhibitor treated mouse OLs and astrocytes in vitro.

Using SAHA concentrations used to test transformed cell susceptibility and even show putative neuroprotective effects, considerable cell death was evident in adult rat OPCs as early as 24 hours following treatment. This was earlier than observed at the identical drugs doses in mouse OPCs (Chapter 3). Furthermore, following 72 hours of treatment, rat OPCs had approximately $20 \%$ less cell survival than mouse OPCs (Chapter 3). Taken together, these data suggest that adult rat spinal cord OPCs are more susceptible to SAHA mediated cell death than mouse OPCs. As proliferative cells are susceptible to HDACi mediated cell death, the increased rat OPC cell death seen following SAHA administration may be due to the increased in vitro proliferative potential of rat OPCs relative mouse OPCs (data not shown).

HDACi has been previously described to cause DNA double strand breaks in both transformed cells and non-transformed human fibroblasts and is a common finding in HDACi treated cells (Lee et al., 2010). Combinatorial treatment of an HDACi with a DNA damaging chemotherapeutic agent is typically seen in order to enhance transformed cell death (Bolden et al., 2006; Marks, 
2010). We do not see a synergistic increase in rat OPC death when SAHA treatment is combined with etoposide. However, with SAHA alone, as early as 6 to 24 hours following treatment rat OPCs have numerous $\mathrm{yH} 2 \mathrm{AX}^{+}$foci, indicating extensive DNA damage likely in the form of DNA double strand breaks.

Furthermore, due to the lack of synergism between etoposide and SAHA mediated OPC cell death, the mechanism of DNA DSB induction mediated by SAHA is likely different than that of etoposide.

One possibility is increased oxidative stress secondary to HDACi resulting in accumulated DNA damage over time. This has been previously described where HDACi mediated by multiple HDAC inhibitors, including SAHA, led to increased reactive oxygen species (ROS) generation (Bolden et al., 2006; Frew et al., 2009). As HDACi may change global gene expression patterns, such as the previously demonstrated reduction in the ROS scavenger thioredoxin, this could lead to ROS accumulation and subsequent DNA damage (Butler et al., 2002; Frew et al., 2009; Marks, 2010). Although oxidized bases typically undergo DNA repair that generates DNA single strand breaks (SSB) rather than DSBs, it is still possible that many SSBs may saturate DNA repair mechanisms with remaining SSBs converted to DSBs during DNA duplex unwinding if OPCs are undergoing replication or transcription (Jackson and Bartek, 2009; Hill and Lee, 2010).

A more likely scenario involves HDACi mediated changes on the standard DNA DSB repair machinery in response to endogenous formation of DNA DSBs during DNA replication. Upon DNA DSB induction, Mre11, RAD50, and NBS1 
(MRN Complex) recognize the site(s) of damage and catalyze the activation of ataxia-telangiectasia mutated (ATM) following by $\mathrm{yH} 2 \mathrm{AX}$ foci formation (Abraham and Tibbetts, 2005; Lombard et al., 2005). ATM induces either cell cycle arrest and/or apoptosis via phosphorylation of $\mathrm{CHK} 1 / \mathrm{CHK} 2$ or $\mathrm{p} 53$, respectively (Lombard et al., 2005; Cimprich and Cortez, 2008). Repair mechanisms are then directed into one of two repair pathways: homologous recombination or nonhomologous end-joining (NHEJ) (Lieber, 2008; San Filippo et al., 2008; Jackson and Bartek, 2009). Recent evidence shows reduced expression of members of the MRN complex following HDACi in transformed cells putatively leading to reduced DNA DSB repair capacity and subsequent apoptosis (Lee et al., 2010). However, the same study indicated this was not the case in normal cells (Lee et al., 2010). Though we did not examine changes in MRN complex expression patterns in our rat OPCs, it remains possible that following HDACi there is a reduced capacity to repair spontaneously formed DNA DSBs. These would accumulate over time and with inefficient DNA DSB repair, apoptosis would then follow. We certainly see induction of apoptosis following HDACi as evidenced by p53 stabilization followed by caspase activation. Subsequent p53 activation may be secondary to ATM activation as the presence of $\mathrm{YH} 2 \mathrm{AX}$ is suggestive of active ATM (Goodarzi et al., 2008; Jackson and Bartek, 2009).

Additionally, HDACi prevents post-translational deacetylation of multiple target proteins (Haberland et al., 2009; Marks, 2010). Here we demonstrate drug activity by increased acetylation of histone $H 3$. Successful DSB repair relies on open, accessible chromatin (euchromatin) in order for repair enzymes to load 
adjacent to DSBs (Misteli and Soutoglou, 2009). This should not present as a problem in an environment of hyperacetylated histones as this is indicative of chromatin in a "relaxed" state. However, HDAC substrates are many and may include specific DNA DSB repair machinery (Misteli and Soutoglou, 2009; Miller et al., 2010). In human cell lines, HDAC4 has been shown to be a critical component of the DNA DSB repair in response to yirradiation or etoposide (Karagiannis et al., 2007). Additionally, SMRT-HDAC3 complex was shown to be necessary for proper DSB repair in mammalian cell lines (Bhaskara et al., 2010). Furthermore, in saccharomyces cerevisiae, though valproic acid-mediated HDACi was not toxic to cells on its own, when combined with an ultravioletmimetic drug, HDAC inhibition prevented deacetylation of Sae2 (human ortholog to CtIP). This leads to accumulation of an acetylated form of Sae2 preventing its interaction and subsequent displacement of Mre11 from the DSB region for proper DSB repair and promoting cell autophagy (Robert et al., 2011). Therefore, it remains possible HDACi in OPCs results in inefficient DNA DSB repair in response to spontaneous DSBs, ultimately leading to cell death.

Unexpectedly, cell death was seen in differentiated rat OLs as well. To our knowledge, this is the first description of extensive HDACi-alone mediated cell death of fully differentiated cells. The in vitro differentiation paradigm we employed results in cell cycle exit and full differentiation of primary rat OPCs (Cao et al., 2010). Furthermore, they were positive for multiple mature $\mathrm{OL}$ markers and had not expanded in culture during the 7 days in differentiation media. Therefore, there is no reason to indicate they continued to retain any form 
of proliferative capacity. We further show OL death to be due in part to p53 mediated induction of caspase-dependent apoptosis as is the case in OPCs as well. Though we do not have direct evidence, the similarity of cell death suggests this p53 mediated induction of apoptosis may be due to DNA damage. As DNA replication is not occurring in rat OLs, inefficient repair of endogenous DNA DSB accumulation during transcription may promote $\mathrm{OL}$ apoptosis. However, the specific mechanism(s) remain to be fully elucidated.

Overall, these results necessitate strict caution in the clinical use of HDAC inhibitors. Though many positive results suggest great therapeutic beneficence from HDACi therapy, these appear to be a model and subsequent end-point driven conclusions. OPCs are absolutely necessary for the normal development of the myelinated mammalian CNS (Knobler et al., 1974; Wolswijk and Noble, 1989; Noble et al., 1992; Miller and Mi, 2007). Furthermore, OL and myelination turnover in adult mammals are only beginning to become better understood (Fields, 2008; Ullen, 2009). As this cytotoxicity also extends to adult OPCs, we have yet to determine if depletion of this cellular population could be detrimental to the diseased, injured, or aging CNS. Furthermore, these data present an immediate concern for patients who are currently undergoing this form of treatment. SAHA is blood-brain-barrier (BBB) penetrable and may contribute to an as of yet unknown cognitive or motor impairment. Therefore, further study is necessary to define HDACi-mediated normal cell death and increase awareness of OPC and OL susceptibility to current and developing chemotherapeutic agents. 


\section{CHAPTER 5}

\section{DISCUSSION}

\section{HDAC INHIBITOR TOXICITY AND CICI}

\section{Clinical profile of HDAC inhibitor induced toxicity}

Current clinical use of HDAC inhibitors as chemotherapeutic agents have revealed a toxicity profile that are somewhat common among other chemotherapeutic drugs, particularly as it relates to detrimental effects on normal, non-transformed proliferative cells. These toxicities examined thus far are short and mainly reversible as widespread use of HDAC inhibitors are still somewhat limited to advanced malignancies that often are accompanied by a reduced time of patient survival (Bruserud et al., 2007). However, with current studies suggesting small molecule HDAC inhibitor therapy may extend to cancers that may have a longer period of patient survival and to non-cancer treatment such as use in neurological disorders, it becomes critical to investigate as of yet undefined toxicities that may exist with HDAC inhibitor treatment.

The two most common toxicities observed with HDAC inhibitor therapy are thrompocytopenia and neutropenia. This appears to be regardless of the chemical structure of the HDAC inhibitor as this is seen with SAHA, depsipeptide, 
and MS-275 (Marshall et al., 2002; Sandor et al., 2002; Kelly et al., 2003; Kelly et al., 2005; Ryan et al., 2005; Bruserud et al., 2007). Nonetheless, both thrombocytopenia and neutropenia secondary to HDAC inhibitor therapy are reversible and short-lived as recovery to normal values occurs quickly following cessation of therapy or may be accompanied following putative dose reduction (Bruserud et al., 2007). Additionally, HDAC inhibitor effects on normal immunocompetent cells account for small molecule HDACi induced immunomodulatory effects (Bruserud et al., 2007). An extensive review discusses the importance of regulation of histone acetylation status in $\mathrm{T}$ cell differentiation and rearrangement of the T cell receptor chains. HDAC inhibitor induced deviations in this regulation that can lead to immunosuppresion may not only influence risk of infection concurrent with chemotherapy but also increase risk of cancer relapse or progression following chemotherapy if lymphoid reconstitution is delayed (Bruserud et al., 2007).

Gastrointestinal side effects (as are typical with many chemotherapeutic agents) are also observed with HDAC inhibitor therapy. These include nausea, diarrhea, and dyspepsia depending on the HDAC inhibitor (i.e. diarrhea reported with depsipeptide and MS-275) and therapeutic dose used (Marshall et al., 2002; Sandor et al., 2002; Kelly et al., 2003; Kelly et al., 2005; Ryan et al., 2005; Bruserud et al., 2007). Among the reported adverse effects following HDAC inhibitor treatment, cardiac effects have also been observed. Some patients display electrocardiogram (ECG) abnormalities as well as dyspnea, though the latter was seen without the presence of other cardiopulmonary symptoms (Kelly 
et al., 2003; Kelly et al., 2005; Bruserud et al., 2007). Though not understood and severely limited in study, neurological toxicity has also been observed following HDAC inhibitor treatment. This includes decreased concentration, coordination, and confusion with treated patients not returning to baseline performance until several weeks following therapy cessation (Gilbert et al., 2001; Bruserud et al., 2007).

\section{Chemotherapy induced cognitive impairment $(\mathrm{CICl})$}

The potential for neurological toxicity following small molecule-mediated $\mathrm{HDACi}$ is of considerable importance. As discussed in Chapter $1, \mathrm{ClCl}$ is only recently beginning to be appreciated clinically although mechanisms involved in its pathogenesis still remain unclear. Of the putative mechanisms that may be involved in $\mathrm{ClCl}$ pathogenesis, direct or indirect DNA damage may be the most relevant due to the importance of maintaining genomic integrity and the number of chemotherapeutic agents that induce DNA damage (Ahles and Saykin, 2007). Normal, non-transformed cells have numerous mechanisms to repair DNA depending on the form of DNA damage that is present. Oxidative stress is a regular source for DNA damage to all cells, particularly those of the CNS (Abner and McKinnon, 2004; Caldecott, 2004; Ahles and Saykin, 2007; Fishel et al., 2007). Chemotherapeutic drugs, such as alkylating agents, induce DNA damage in a number of transformed cell types, however, a number of studies have found this damage extends to the DNA of healthy cells. Peripheral blood lymphocytes show evidence of DNA damage and reduced DNA repair efficacy in patients relative to controls, however, this was found to be the true before and after 
chemotherapy treatment (Blasiak et al., 2004; Nadin et al., 2006; Ahles and Saykin, 2007). As chemotherapy is associated with an attenuated antioxidant capacity and increased production of free oxygen radicals, DNA damage secondary to oxidative stress in normal healthy cells should be anticipated and is likely a strong candidate for $\mathrm{ClCl}$ development secondary to DNA damage in post-mitotic neurons (Kaya et al., 2005; Papageorgiou et al., 2005; Ahles and Saykin, 2007; Fishel et al., 2007).

\section{HDAC inhibitor mediated cytotoxicity in normal cells}

In addition to the previously discussed downstream biological effects following $\mathrm{HDACi}$, two are of great relevance in the context of DNA damage and genomic integrity (Chapter 1). Treatment with various HDAC inhibitors results in increased reactive oxygen species (ROS) generation with the addition of freeradical scavengers attenuating tumor cell death (Bolden et al., 2006). Increased cellular ROS precedes mitochondrial disruption suggesting that increased ROS generation is not merely an event secondary to mitochondrial damage (Bolden et al., 2006; Frew et al., 2009; Marks, 2010). Though still not entirely defined, increase intracellular ROS appears to be secondary to increased expression of thioredoxin binding protein- 2 (TBP-2), a negative regulator of thioredoxin activity and expression. Increased TBP-2 leads to increased inhibition of the ROS antioxidant scavenger reduced Thioredoxin resulting in increased ROS (Butler et al., 2002; Ungerstedt et al., 2005; Frew et al., 2009; Marks, 2010). Ultimately, increased intracellular ROS will lead to DNA damage, and protein and lipid oxidation ultimately leading to apoptosis. 
HDAC inhibitors are often co-administered with other chemotherapeutic agents in order to enhance transformed cell death. For instance, when administered in conjunction with chemotherapeutic DNA damaging agents, such as cisplatin or etoposide in various human cancer cell lines, they act synergistically to enhance tumor cell death compared to any of the agents used alone (Kim et al., 2003; Frew et al., 2009; Shiozawa et al., 2009). Nonetheless, small molecule-mediated HDACi on its own has been demonstrated to cause DNA damage as well. HDACi's do not appear to do this directly, but it has been suggested HDACi mediated chromatin structural changes result in greater DNA exposure to damaging agents such as HDACi induced ROS (Marks, 2010). However, HDACi mediated disruption of DNA repair pathways leading to accumulation of putatively ROS induced DNA damage and subsequent apoptosis, is more likely to contribute to HDACi mediated DNA damage rather than the increased the euchromatin modification that ensues HDACi treatment. HDACi treatment results in the accumulation of $\mathrm{yH} 2 \mathrm{AX}$, a marker for DNA DSBs (Chapter 1). Furthermore, regulation of post-translation deacetylation of DNA repair proteins by HDACs play pivotal role in the successful execution of DNA repair (Chapter 1). Additionally, HDACi mediated transcriptional changes may play a role in DSB accrual (Chapter 1). HDACi reduces expression of genes encoding DNA repair proteins involved in HR and NHEJ mediated DNA DSB repair, such as RAD51, BRCA1, BRCA2, Ku70, Ku86, and DNA-PK s, RAD50, and MRE11 (Frew et al., 2009; Lee et al., 2010; Marks, 2010). DNA DSB accumulation ovenwhelms repair pathways resulting in cell cycle arrest and/or 
apoptosis, implicating perturbation of DNA DSB repair pathways as a critical component of HDACi mediated cell death.

Though extensive work has gone into identifying therapeutic uses of HDAC inhibitors in cancer treatment, particularly as it relates to mechanisms of cytotoxicity towards a wide range of transformed human cell lines, studies investigating their toxicity towards normal cells with the potential of explaining clinically observed adverse effects are relatively limited (Fig. 29). Nonetheless, they are highly relevant, effect a variety of normal proliferative cells, and require further investigation to reexamine the therapeutic benefits and limits of HDACi therapy. Among the first studies of this nature, small molecule mediated HDAC inhibition by SAHA and TSA demonstrated normal renal tubular cell toxicity. At in vitro SAHA concentrations that have previously been extrapolated to have toxicity towards cancer cell lines induced apoptosis in rat renal proximal tubular cells in both caspase-dependent and independent mechanisms. Furthermore, this appears to be via a p53-independent mechanism and related to a reduction in anti-apoptotic protein levels (Dong et al., 2008). This provides a putative mechanism for renal cell toxicity and the clinically observed, though uncommon, renal insufficiency following HDAC inhibitor treatment (Bruserud et al., 2007; Dong et al., 2008).

This was followed by a number studies demonstrating HDAC inhibitor cytotoxicity of human mesenchymal lineage stem cells (MSCs). The pan-HDAC inhibitor, SAHA, and the HDAC1/class I HDAC inhibitor, MS-275 resulted in cell cycle arrest and subsequent apoptotic induction following treatment of human 
MSCs isolated from healthy bone marrow donors (Di Bernardo et al., 2009). Furthermore, adipose and cord blood derived human MSCs treated with HDAC inhibitors valproic acid (VPA) and sodium butyrate resulted in cell cycle arrest and reduced their efficiency to differentiate into multiple cell types such as adipocytes, chondrocytes, and neurons (Lee et al., 2009). Lastly, multiple studies have shown decreased bone density and increased fracture risk in animal models treated with SAHA or VPA (Nissen-Meyer et al., 2007; Pratap et al., 2010; McGee-Lawrence et al., 2011). In fact, prolonged treatment in humans with VPA reduced bone density in children and adults (Sheth et al., 1995; Boluk et al., 2004; McGee-Lawrence et al., 2011). To delve closer into these findings, a recent study of HDAC inhibitor-induced toxicity examined the effect $\mathrm{HDACi}$ has on maintenance of bone mass. As reported previously, in vivo administration of SAHA into adult mice significantly reduced bone density. Corroborative in vitro studies strongly suggest this loss is due to SAHA-mediated reduction of immature osteoblast number (McGee-Lawrence et al., 2011). Overall, these findings demonstrate small molecule-mediated HDACi cytotoxicity may deplete the reservoir of bone marrow derived MSCs and furthermore, lead to detrimental changes in skeletal development and maintenance (Di Bernardo et al., 2009; Lee et al., 2009; McGee-Lawrence et al., 2011).

As it became clear that HDACi may have off-target effects towards normal, non-transformed cells, the question remained as to how this cytoxicity is mediated. Additional work revealed that it is considerably similar to small molecule-mediated HDACi on cancerous cells. In vitro studies demonstrated an 
increase in cell death and/or senescence when HDAC inhibitor treatment was combined with UV irradiation or $\mathrm{H}_{2} \mathrm{O}_{2}$ of MSCs (Di Bernardo et al., 2010). Immunocytochemical analyses of $\mathrm{H}_{2} \mathrm{O}_{2}$ exposed MSCs when combined with SAHA or MS-275 treatment showed a significant increase in the number of cells immunpositive for the DNA oxidative damage marker 8-oxo-7,8-dihydroguanine (8oxodG) relative to $\mathrm{H}_{2} \mathrm{O}_{2}$ alone (Di Bernardo et al., 2010). The aforementioned study regarding increased bone loss due to reduced osteoblast number showed SAHA treatment alone of bone marrow stromal cells (BMSCs) was sufficient to cause DNA damage as demonstrated by an increase in the percentage of YH2AX-positive nuclei (McGee-Lawrence et al., 2011). Lastly, though using relatively higher concentrations, a recent study demonstrated increased cell death following an increased induction of DNA double-strand breaks (DSBs) as indicated by the number of $\mathrm{yH} 2 \mathrm{AX}$-foci per cell when primary human skin fibroblasts were pre-treated with HDAC inhibitors followed by irradiation versus irradiation alone (Purrucker et al., 2010). However, seemingly conflicting with the Purrucker et al. study, another investigation demonstrated normal human fibroblasts are able to repair DSBs following SAHA treatment that transformed cell lines cannot repair, hence establishing a preferential susceptibility of transformed cell lines to SAHA- mediated HDACi (Lee et al., 2010). Nonetheless, there is sufficient evidence to suggest small molecule mediated HDACi may result in cytotoxicity towards healthy cells such as MSCs and cell cycle arrest of BMSCs, it remains to be seen whether it is sufficient to overcome normal cellular DNA repair mechanisms in many non-transformed cell types (Fig. 29). 


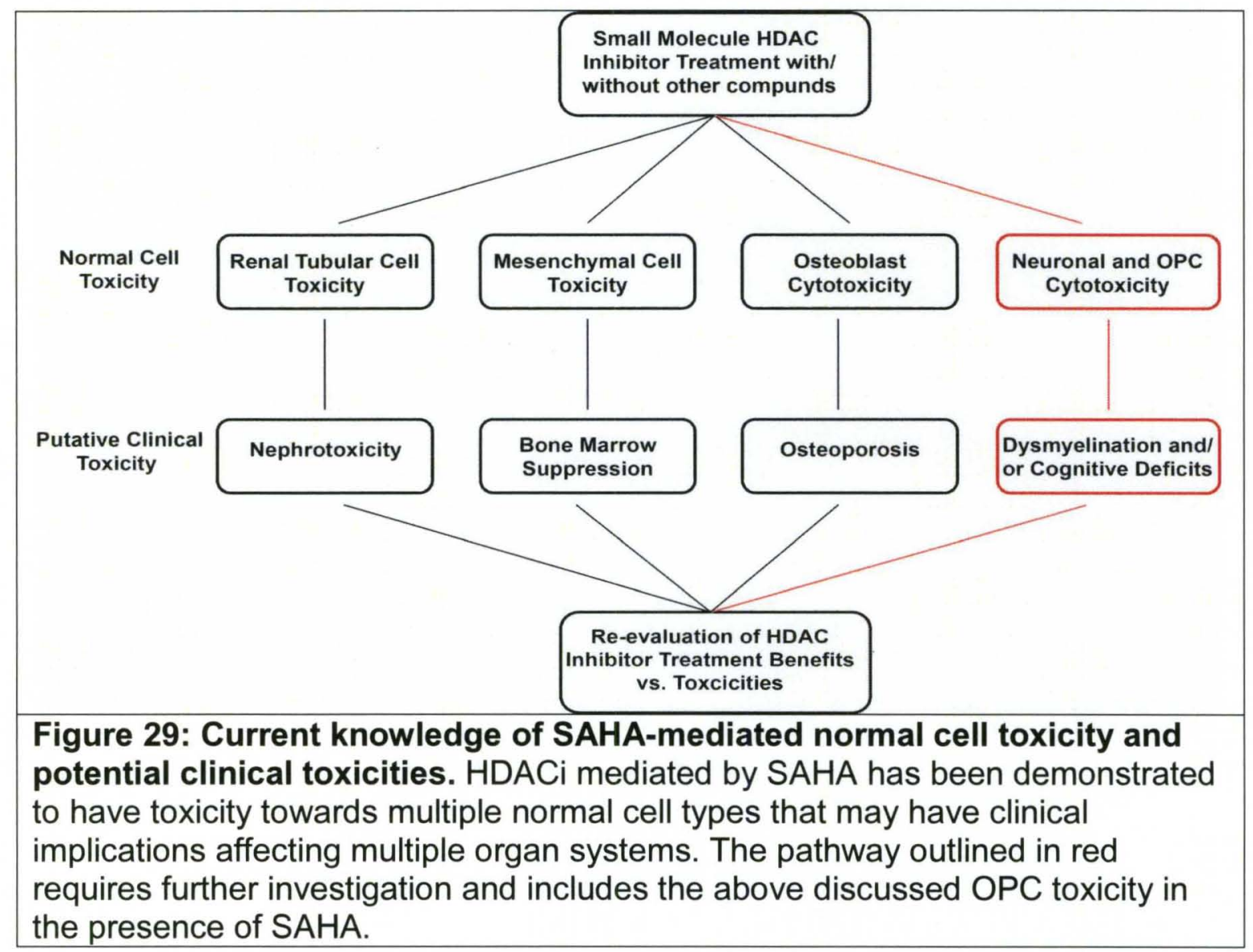

\section{Cytotoxic effects of small molecule-mediated HDACi on OLs}

As discussed in chapter 1, HDAC activity plays a critical role in regulating OPC maturation into mature myelinating OLs, whereby HDACi prevents OPC maturation into OLs and may even promote their differentiation towards a neuronal lineage (Shen and Casaccia-Bonnefil, 2008; Copray et al., 2009; Dietz and Casaccia, 2010). The experimental evidence described herein go a step further and demonstrate small molecule-mediated HDACi alone at concentrations at and below those extrapolated for use against a variety of cancers causes increased cortically-derived mouse postnatal OPC cell death both in vitro and in vivo. Furthermore, this is at least in part due to increased caspase-dependent 
apoptosis and is secondary to an induction of widespread DNA DSBs observed as early as six hours following SAHA treatment in adult spinal cord-derived rat OPCs. However, the effect of SAHA administration in vitro on mature OLs is not entirely clear. Differentiated mouse OPCs did show significant increase in cell death following SAHA treatment but these may have been cells that had yet to fully mature and therefore, may have still been susceptible to SAHA-mediated cytotoxocity. Despite this, rat OPCs that were permitted to differentiate into fully mature OLs remained susceptible to SAHA-induced cytotoxicity (Chapter 4).

Though, as far as we are aware, this is the first study that demonstrates HDACi treatment resulting in accumulation of DNA DSBs with subsequent cell death described in the oligodendroglial lineage, detrimental effects of chemotherapy induced dysmyelination and $\mathrm{OL}$ death have been previously described. Systemic administration of chemotherapeutic agents carmustine, cytosine arabinoside, and cisplatin were shown to illicit cytotoxicity towards CNS progenitor cells, including OPCs, in multiple regions of the mouse brain (Dietrich et al., 2006). Furthermore, intraperitoneal administration of 5-flurorouracil (5-FU), a chemotherapeutic agent used comprehensively in the treatment of colorectal cancer as well as an adjuvant therapy in a wide range of other cancers, caused considerable OL cell death in vitro and in vivo. Additionally, as late as seven weeks following 5-FU administration, extensive myelinopathy accompanied by reduced cellularity were observed by ultrastructural and immunohistochemical analyses of the corpus callosi of adult mice (Han et al., 2008). These ultimately corroborated clinical data demonstrating white matter abnormalities and cognitive 
impairments in patients receiving treatment with these chemotherapeutic agents providing strong evidence of the involvement of $\mathrm{OL}$ loss in $\mathrm{ClCl}$ pathogenesis.

\section{DUALISM OF HDAC INHIBITION}

As extensively reviewed and previously discussed in chapter 1 , small molecule-mediated HDACi have also been recently identified as being beneficial in a number of neurological disease models. Briefly, these include neurodegenerative diseases such as Parkinson's and Huntington's disease, Rubenstein-Taybi syndrome, Rett syndrome and ischemic stroke models (Abel and Zukin, 2008; Kazantsev and Thompson, 2008; Colussi et al., 2010). Additionally, studies investigating the use of HDAC inhibitors for the treatment of psychiatric disorders such as schizophrenia, bipolar disorder, and depression have also suggested their use to improve disease outcome in animal models (Abel and Zukin, 2008; Grayson et al., 2010). More specifically, it was recently demonstrated that mouse intra-hippocampal administration of the HDAC inhibitor MS-275 promoted anti-depressant like activity such as reversal of defeat-induced deficit of sucrose preference and lessening in social avoidant behavior (Covington et al., 2011). Lastly, animal models of neuromuscular diseases such as amyotropic lateral sclerosis (ALS), spinal muscular atrophy (SMA), and Friedreich's ataxia treated with HDAC inhibitors have also demonstrated some degree of therapeutic beneficence (Abel and Zukin, 2008; Kazantsev and Thompson, 2008; Colussi et al., 2010). 
However, a relevant question necessarily follows: How do we consolidate the extensive experimental evidence demonstrating the cytotoxic effects of HDAC inhibitors with its neuroprotective and neurotrophic effects in a multitude of disease models, most notably of those being HDAC inhibitor treatment of neurological disorders? Often times these differences are attributed to drug dosage and/or delivery variability. However, in the case of HDAC inhibitors experimental treatment paradigms showing both cytotoxic and cytoprotective effects have been relatively similar. Differences may also be explained by the inability of transformed cells to overcome the induction of DNA damage by HDAC inhibitors, whereas normal cells are either resistant and/or have effective DNA reparative processes in place thereby allowing the demonstration of HDAC inhibitors cytoprotective properties. However, as discussed above, normal cells are susceptible to small molecule-mediated HDACi cytotoxicity at concentrations comparable, if not less than those of cancerous cells (Bruserud et al., 2007; Dong et al., 2008; Di Bernardo et al., 2009; Lee et al., 2010; McGee-Lawrence et al., 2011). Perhaps the variation of HDACi on proliferative versus differentiated cells may explain this difference in cellular effect. Actively dividing cells may be more susceptible to DNA damaging agents such as HDAC inhibitors and thereby are overcome by its cytotoxic induction whereas differentiated cells, such as neurons, can withstand this. However, preliminary evidence suggests that at least rat mature OLs are as susceptible to HDACi mediated cytotoxicity as their proliferative OPC counterparts (Chapter 4). Additionally, rat cortical neurons treated with both an HDAC inhibitor and a DNA damaging agent topoisomerase II 
inhibitor, etoposide, result in extensive cytotoxicity (unpublished data, privileged communication with Dr. Michal Hetman).

Rather than searching for differences in the status of their targets, emphasis needs to placed on the direct downstream effects that follow HDACi that are likely similar amongst many different cell types, normal or transformed. Similar mechanisms have been described to explain both the cytotoxic and cytoprotective effects of HDAC inhibitors. They involve changes in gene transcription and post-translational modification of protein acetylation status (Colussi et al., 2010; Marks, 2010). These two mechanisms are also linked as post-translational modification of trans-acting factors and/or of histones lead to changes in chromatin structure that may directly alter gene transcription. Such changes include increased expression of pro-apoptotic genes and death receptors and ligands in transformed cells, but also increased expression of target disease genes normally silenced in the disease phenotype, such as the frataxin gene in Friedreich's ataxia (Kazantsev and Thompson, 2008; Marks, 2010). However, changes in acetylation status of proteins also affect proteins that are not involved in transcription but are critical to normal cell function and survival. These, for example, include regulation of microtubule dynamics specifically by HDAC6, stabilization of $p 53$, and modification of DNA repair pathways (Kazantsev and Thompson, 2008; Misteli and Soutoglou, 2009; Miller et al., 2010). Yet, often studies demonstrating protective effects following $\mathrm{HDACi}$ are largely endpoint driven whereby they may not experimentally investigate known downstream cytotoxic effects of HDACi therapy. 
Therefore, to further understand this incongruity in the outcome of HDAC inhibitor treatment it is perhaps important to appreciate a critical concept: Compounds that modify global cellular processes invariably have global cellular consequences. However, the term global does not refer to transcriptional changes as numerous studies indicates that $\mathrm{HDACi}$ results in transcriptional expression changes that effect subsets of genes (Kazantsev and Thompson, 2008; Thomas, 2009; Colussi et al., 2010). Rather, although HDAC inhibitors alter the balance of expression between pro-survival and pro-apoptotic genes seemingly in favor of one or the other depending on the question being investigated, they too are likely affecting other downstream processes that have been previously described in other studies such as DNA repair efficiency, reactive oxygen species (ROS) generation, and/or cell cycle arrest that may likewise be cytotoxic to otherwise healthy cells not central to the experimental question.

Furthermore, the effects of HDACi will depend on the specific cell population being investigated as systemic HDAC inhibitor administration in experimental animal models and humans exposes the majority, if not all cell types- normal, diseased, or transformed- to their putative downstream effects. As discussed above, normal cell types such as renal tubular cells and MSCs are susceptible to HDAC inhibitor induced apoptosis. Also as discussed above, this furthermore extends to OPCs, where either cell cycle arrest may occur preventing differentiation into mature OLs or induction of apoptosis following DNA DSB formation (Chapters 1, 3, \& 4). 
Additionally and likely driving some degree of the cell specific $\mathrm{HDACi}$ responses observed are that the variety of effects mediated by HDAC inhibitors may simply be a consequence of which HDAC isoforms are normally expressed in different tissues and cell types. In fact, many studies as discussed by a recently published review have revealed that even in the CNS, HDAC isoform expression patterns vary considerably amongst many brain regions (i.e. cerebellum, striatum, amygdala) and the spinal cord (Thomas, 2009). Needless to say, this offers another level of complexity of HDACi treatment that could explain its divergent effects. A recent study demonstrated that primary ventral midbrain neurons are susceptible to caspase-independent apoptosis following HDAC inhibitor treatment. They also found that this toxicity did not extend to primary cortical neurons (Forgione and Tropepe, 2011). This difference in cytotoxicity could putatively be explained either by varying HDAC isoform expression patterns between the two neuronal populations, though this possibility has yet to be investigated.

Lastly, differences in HDAC expression, even within the same cell type, also likely depend on the cell context- whether it is functioning normally, stressed, or dying. This likely depends on the cellular environment at that time of HDAC inhibitor administration. To illustrate this point, there are a number of studies largely originating from one group that have defined OPC cell cycle arrest or perhaps even loss of glial lineage restriction upon differentiation with $\mathrm{HDACi}$ (Chapter 1). We took this story a step further with experimental evidence demonstrating HDACi results in OPC DNA damage with subsequent cell death in 
vitro and in vivo. However, a recent publication clearly shows white matter preservation in an ex vivo prep under ischemic conditions with small moleculemediated HDACi (Baltan et al., 2011). We can reconcile the two contrasting results by considering the cellular context at the time of HDACi- where cytotoxicity is present following HDACi in healthy, normal functioning OPCs whereas cytotoxicity may be attenuated if OPCs are in the presence of a greater stress than $\mathrm{HDACi}$, such as ischemia.

Overall, non-transformed cell types are just as susceptible, if not more, to HDACi mediated cytotoxicity relative to cancer cells as evidenced by its effect at relatively low drug concentrations on MSCs, osteoblasts, and OPCs (Di Bernardo et al., 2009; McGee-Lawrence et al., 2011). Yet, a large number of studies and reviews have clearly demonstrated the neuroprotective effects of HDACi despite a minority of findings suggesting HDAC inhibitors may be neurotoxic. What drives these differing susceptibilities and responses to HDACi, particularly in healthy non-transformed CNS cells are likely to be multifactorial and require much needed investigation to both limit normal cell cytotoxicity while specifically enhancing HDAC inhibitors' putative protective effects.

\section{FUTURE HDAC INHIBITOR DEVELOPMENT AND THERAPY}

As the evidence for therapeutic use of HDAC inhibitors increases with a concomitant increase in the number of clinical trials using these drugs in an increasing variety of diseases, fully understanding toxicities affiliated with $\mathrm{HDACi}$ is of great importance. The general direction of HDAC inhibitor development is 
towards discovery and synthesis of HDAC isoform selective compounds (Thomas, 2009; Bertrand, 2010). Though this is proposed to likely reduce nonspecific effects, particularly towards healthy cells, greater evidence is still needs to be demonstrated that isoform selective inhibition is sufficient.

With the data presented in the above chapters as well as slowly increasing evidence of HDAC inhibitor cytotoxicity towards normal, non-transformed cells, I propose two additional measures need to be taken as well. Assess clinical use, safety, and promote future development of HDAC inhibitors that have little to no BBB penetrability. Currently, HDAC inhibitor development is trying to increase BBB penetrability for use against CNS tumors and neurodegenerative conditions. However, with the possibility that the scientific community is being a somewhat hasty in progressing HDAC inhibitors into the clinical trials for use in neurodegenerative conditions and traumas, HDAC inhibitors in use against a variety of malignancies that only remains in the periphery would spare any as of yet fully defined CNS toxicities while still maintaining their well documented efficacy against a peripheral malignancy. This would allow us time to understand the limits of HDAC inhibitor therapy in the context of CNS disease while providing oncologists and cancer patients with a potential new drug that may increase survivability.

Secondly, there needs to be a greater appreciation amongst the basic neuroscience community regarding both the implications and relevance of investigating CNS neurotoxicity secondary to chemotherapeutic agents. Cognitive impairment prior to, during, and following chemotherapy is being better 
assessed clinically than in years past (Argyriou et al., 2010; Janelsins et al., 2011). Yet it appears neuroscientists investigating this phenomenon are still considerably limited. Due in large part to the amount of funding and societal need, cancer research is expansive leading to a seemingly uninterrupted flow of rapid developments, synthesis of new drugs, and novel treatment strategies. With now an improved appreciation for the significance of $\mathrm{ClCl}$ development, I would like to see the neuroscience community dramatically increase its efforts investigating CNS toxicities of new chemotherapies as they are developed in basic science labs.

As cancer therapies improve and the number of cancer survivors continues to increase in the ensuing years, we need to no longer rely solely on patient's cognitive tests and imaging studies after drug treatment as effects may be irreversible. Rather, putative neurotoxicity and oligodendrotoxicity profiles of old and new chemotherapeutic drugs will be determined prior to clinical trials which would limit or entirely prevent exposure to CNS toxic agents of which may not have sufficient benefit to warrant clinical use. This too would have the added benefit of increasing our knowledge on what biological processes (as they are altered by chemotherapeutic agents) CNS cells may either be tolerant/ resistant or susceptible to. With the potential of being applicable to other subspecialties of neuroscience research, such information may greatly further our understanding of other neurological disorder models. 


\section{SUMMARY}

\section{$\mathrm{HDACi}, \mathrm{CICl}$ pathogenesis, and concluding remarks}

Despite future directions of HDAC inhibitor drug development that have greater HDAC isoform selectivity resulting in cellular specific affects that decrease potential toxicities, previously developed HDAC inhibitors, most notably SAHA, are currently in use as a chemotherapeutic agent and may have unknown adverse CNS toxicities (Fig. 30). For SAHA alone (trade name Vorinostat $\circledast$, Zolinza () , over 180 clinical trials are listed-and the number increases dramatically when including clinical trials for other HDAC inhibitors such as MS275 (trade name Entinostat $\AA$ ). Furthermore, with extensive experimental evidence indicating cytoprotective effects of HDACi therapy in disease models that include neurological and psychiatric disease, cardiovascular disease, and autoimmune disorders, there already is an endorsement for administration of HDAC inhibitors for treatment in non-cancer related illnesses. Therefore, it becomes more pressing than ever to fully understand the CNS toxicities that are associated with small-molecule- mediated HDACi. 


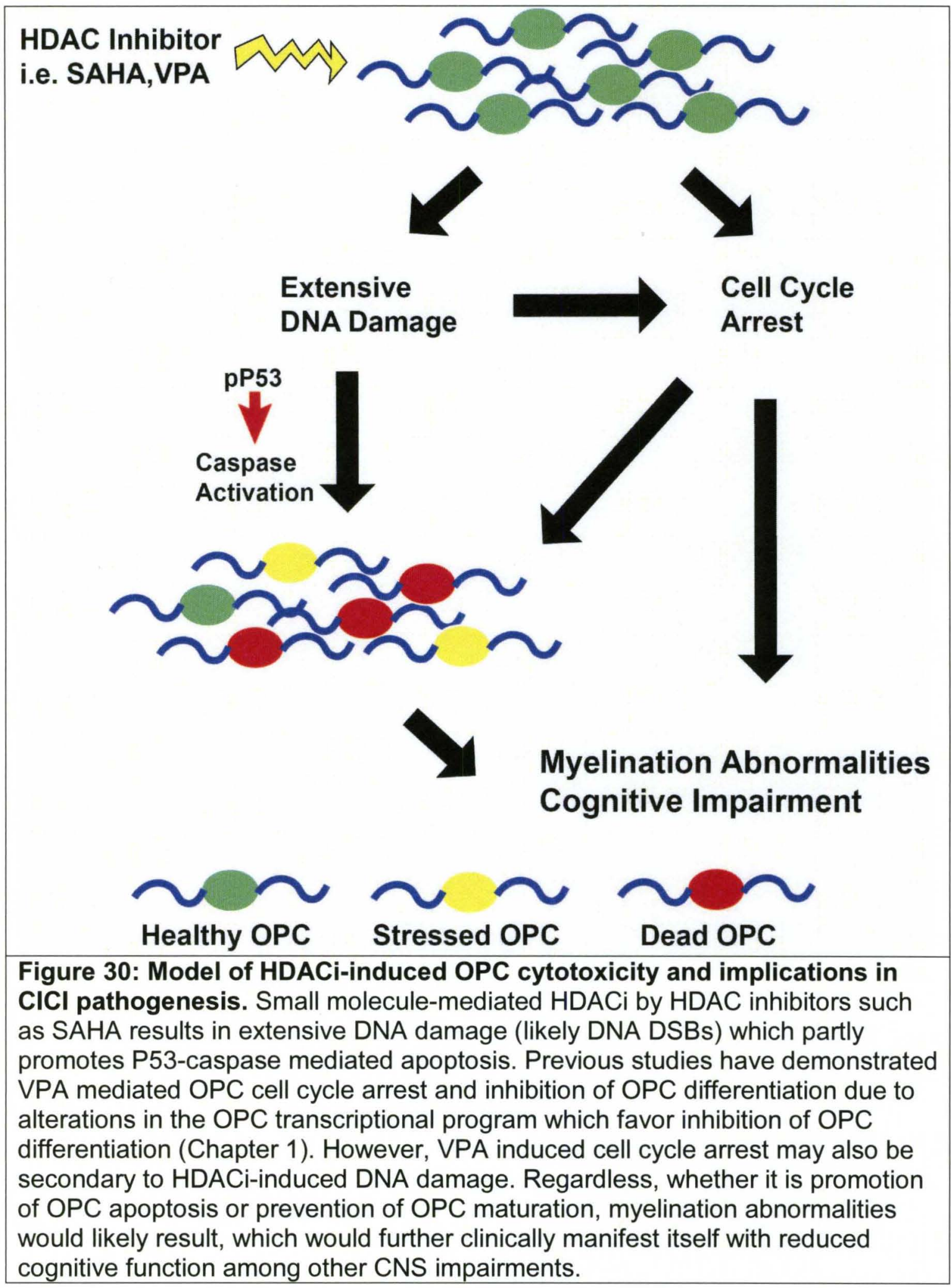


As HDACi-mediated cytotoxicities are clearly evident in neurons as well as OLs, and more specifically OPCs, it's necessary to assess if HDACi in patients currently receiving therapeutic $\mathrm{HDAC}$ inhibitors result in any $\mathrm{ClCl}$ induction (Fig. 30). Though not given nearly as much attention as their neuronal counterparts, OPCs and mature OLs have a critical role in $\mathrm{CICl}$ pathogenesis that has yet to be fully defined and appreciated. Many studies and thorough reviews have illustrated the importance of myelination and white matter normality as it relates to axonal integrity, learning, memory, and overall cognitive function (Fields, 2008; Nave, 2010). Furthermore, the studies described herein in addition to the previously discussed studies assessing the affects of a number of chemotherapeutic agents such as cisplatin and 5-FU reveal that $\mathrm{OL}$ death and white matter abnormalities may manifest either acutely or in a delayed fashion following chemotherapeutic drug administration and therefore contribute to progressive $\mathrm{ClCl}$ development over a large time course (Han et al., 2008; Meyers, 2008).

As the use of HDAC inhibitors for a large variety of malignancies will likely increase over the coming years, it becomes necessary to clearly characterize all cytotoxicities that occur due to its use- particularly its effects on the CNS. However, concerns over CNS toxicity have remained largely marginalized by the numerous reports demonstrating the neuroprotective potential of HDAC inhibitors in an assortment of neurological disorders. Consolidating the two roles these class of drugs play- cytotoxicity versus cytoprotection- when drug concentration, administration, and duration methods are equal is a relatively complex 
undertaking and depends on a number of factors such as cell lineage, cell context and environment, and whether the cell is proliferative or fully differentiated. Therefore, thoroughly investigating the role HDACi has on survival of cells within the oligodendroglial lineage will not only shed insight on new drugs and mechanisms involved in $\mathrm{ClCl}$ pathogenesis but further our understanding on the significant role OL survival and myelin maintenance have on cognitive function. 


\section{REFERENCES}

Abel T, Zukin RS (2008) Epigenetic targets of HDAC inhibition in neurodegenerative and psychiatric disorders. CurrOpinPharmacol 8:5764.

Abner CW, McKinnon PJ (2004) The DNA double-strand break response in the nervous system. DNA Repair (Amst) 3:1141-1147.

Abraham J, Haut MW, Moran MT, Filburn S, Lemiuex S, Kuwabara H (2008) Adjuvant chemotherapy for breast cancer: effects on cerebral white matter seen in diffusion tensor imaging. Clin Breast Cancer 8:88-91.

Abraham RT, Tibbetts RS (2005) Cell biology. Guiding ATM to broken DNA. Science 308:510-511.

Ahles TA, Saykin AJ (2002) Breast cancer chemotherapy-related cognitive dysfunction. Clin Breast Cancer 3 Suppl 3:S84-90.

Ahles TA, Saykin AJ (2007) Candidate mechanisms for chemotherapy-induced cognitive changes. Nat Rev Cancer 7:192-201.

Anderson-Hanley C, Sherman ML, Riggs R, Agocha VB, Compas BE (2003) Neuropsychological effects of treatments for adults with cancer: a metaanalysis and review of the literature. J Int Neuropsychol Soc 9:967-982.

Ankeny DP, Popovich PG (2009) Mechanisms and implications of adaptive immune responses after traumatic spinal cord injury. Neuroscience 158:1112-1121.

Argyriou AA, Assimakopoulos K, Iconomou G, Giannakopoulou F, Kalofonos HP (2010) Either Called "Chemobrain" or "Chemofog," the Long-Term Chemotherapy-Induced Cognitive Decline in Cancer Survivors Is Real. J Pain Symptom Manage.

Avila AM, Burnett BG, Taye AA, Gabanella F, Knight MA, Hartenstein P, Cizman Z, Di Prospero NA, Pellizzoni L, Fischbeck KH, Sumner CJ (2007) Trichostatin $A$ increases SMN expression and survival in a mouse model of spinal muscular atrophy. JClinlnvest 117:659-671. 
Awatramani R, Scherer S, Grinspan J, Collarini E, Skoff R, O'Hagan D, Garbern $\mathrm{J}$, Kamholz J (1997) Evidence that the homeodomain protein Gtx is involved in the regulation of oligodendrocyte myelination. $J$ Neurosci 17:6657-6668.

Baltan S, Murphy SP, Danilov CA, Bachleda A, Morrison RS (2011) Histone deacetylase inhibitors preserve white matter structure and function during ischemia by conserving ATP and reducing excitotoxicity. J Neurosci 31:3990-3999.

Barres BA, Koroshetz WJ, Swartz KJ, Chun LL, Corey DP (1990) lon channel expression by white matter glia: the O-2A glial progenitor cell. Neuron 4:507-524.

Bates EA, Victor M, Jones AK, Shi Y, Hart AC (2006) Differential contributions of Caenorhabditis elegans histone deacetylases to huntingtin polyglutamine toxicity. JNeurosci 26:2830-2838.

Bertrand P (2010) Inside HDAC with HDAC inhibitors. Eur J Med Chem 45:20952116.

Bhaskara S, Knutson SK, Jiang G, Chandrasekharan MB, Wilson AJ, Zheng S, Yenamandra A, Locke K, Yuan JL, Bonine-Summers AR, Wells CE, Kaiser JF, Washington MK, Zhao Z, Wagner FF, Sun ZW, Xia F, Holson EB, Khabele D, Hiebert SW (2010) Hdac3 is essential for the maintenance of chromatin structure and genome stability. Cancer Cell 18:436-447.

Billiards SS, Haynes RL, Folkerth RD, Borenstein NS, Trachtenberg FL, Rowitch DH, Ligon KL, Volpe JJ, Kinney HC (2008) Myelin abnormalities without oligodendrocyte loss in periventricular leukomalacia. Brain Pathol 18:153163.

Billon N, Jolicoeur C, Tokumoto Y, Vennstrom B, Raff M (2002) Normal timing of oligodendrocyte development depends on thyroid hormone receptor alpha 1 (TRalpha1). EMBO J 21:6452-6460.

Blasiak J, Arabski M, Krupa R, Wozniak K, Rykala J, Kolacinska A, Morawiec Z, Drzewoski J, Zadrozny M (2004) Basal, oxidative and alkylative DNA damage, DNA repair efficacy and mutagen sensitivity in breast cancer. Mutat Res 554:139-148.

Bolden JE, Peart MJ, Johnstone RW (2006) Anticancer activities of histone deacetylase inhibitors. Nat Rev Drug Discov 5:769-784. 
Boluk A, Guzelipek M, Savli H, Temel I, Ozisik HI, Kaygusuz A (2004) The effect of valproate on bone mineral density in adult epileptic patients. Pharmacol Res 50:93-97.

Brinkmann BG, Agarwal A, Sereda MW, Garratt AN, Muller T, Wende H, Stassart RM, Nawaz S, Humml C, Velanac V, Radyushkin K, Goebbels S, Fischer TM, Franklin RJ, Lai C, Ehrenreich H, Birchmeier C, Schwab MH, Nave KA (2008) Neuregulin-1/ErbB signaling serves distinct functions in myelination of the peripheral and central nervous system. Neuron 59:581595.

Brown MS, Stemmer SM, Simon JH, Stears JC, Jones RB, Cagnoni PJ, Sheeder JL (1998) White matter disease induced by high-dose chemotherapy: longitudinal study with MR imaging and proton spectroscopy. AJNR Am J Neuroradiol 19:217-221.

Bruserud O, Stapnes C, Ersvaer E, Gjertsen BT, Ryningen A (2007) Histone deacetylase inhibitors in cancer treatment: a review of the clinical toxicity and the modulation of gene expression in cancer cell. Curr Pharm Biotechnol 8:388-400.

Butler LM, Zhou X, Xu WS, Scher HI, Rifkind RA, Marks PA, Richon VM (2002) The histone deacetylase inhibitor SAHA arrests cancer cell growth, upregulates thioredoxin-binding protein-2, and down-regulates thioredoxin. Proc Natl Acad Sci U S A 99:11700-11705.

Butler R, Bates GP (2006) Histone deacetylase inhibitors as therapeutics for polyglutamine disorders. NatRevNeurosci 7:784-796.

Butler RW, Hill JM, Steinherz PG, Meyers PA, Finlay JL (1994) Neuropsychologic effects of cranial irradiation, intrathecal methotrexate, and systemic methotrexate in childhood cancer. J Clin Oncol 12:26212629.

Cahoy JD, Emery B, Kaushal A, Foo LC, Zamanian JL, Christopherson KS, Xing Y, Lubischer JL, Krieg PA, Krupenko SA, Thompson WJ, Barres BA (2008) A transcriptome database for astrocytes, neurons, and oligodendrocytes: a new resource for understanding brain development and function. J Neurosci 28:264-278.

Cai J, Zhu Q, Zheng K, Li H, Qi Y, Cao Q, Qiu M (2010) Co-localization of Nkx6.2 and $\mathrm{NkX2.2}$ homeodomain proteins in differentiated myelinating oligodendrocytes. Glia 58:458-468.

Calaora V, Rogister B, Bismuth K, Murray K, Brandt H, Leprince P, Marchionni $M$, Dubois-Dalcq M (2001) Neuregulin signaling regulates neural precursor 
growth and the generation of oligodendrocytes in vitro. J Neurosci $21: 4740-4751$.

Caldecott KW (2004) DNA single-strand breaks and neurodegeneration. DNA Repair (Amst) 3:875-882.

Cao Q, Xu XM, Devries WH, Enzmann GU, Ping P, Tsoulfas P, Wood PM, Bunge MB, Whittemore SR (2005) Functional recovery in traumatic spinal cord injury after transplantation of multineurotrophin-expressing glialrestricted precursor cells. JNeurosci 25:6947-6957.

Cao Q, He Q, Wang Y, Cheng X, Howard RM, Zhang Y, DeVries WH, Shields CB, Magnuson DS, Xu XM, Kim DH, Whittemore SR (2010) Transplantation of ciliary neurotrophic factor-expressing adult oligodendrocyte precursor cells promotes remyelination and functional recovery after spinal cord injury. J Neurosci 30:2989-3001.

Chen CS, Wang YC, Yang HC, Huang PH, Kulp SK, Yang CC, Lu YS, Matsuyama S, Chen CY (2007a) Histone deacetylase inhibitors sensitize prostate cancer cells to agents that produce DNA double-strand breaks by targeting Ku70 acetylation. Cancer Res 67:5318-5327.

Chen S, Wu H, Ossola B, Schendzielorz N, Wilson B, Chu C, Wang Q, Zhang D, Qian L, Li X, Hong J, Lu R (2011) Suberoylanilide Hydroxamic Acid (SAHA), a Histone Deacetylase Inhibitor, Protects Dopaminergic Neurons from Neurotoxin-Induced Damage. Br J Pharmacol.

Chen Y, Balasubramaniyan V, Peng J, Hurlock EC, Tallquist M, Li J, Lu QR (2007b) Isolation and culture of rat and mouse oligodendrocyte precursor cells: Nat Protoc 2:1044-1051.

Cheng X, Wang Y, He Q, Qiu M, Whittemore SR, Cao Q (2007) Bone morphogenetic protein signaling and olig1/2 interact to regulate the differentiation and maturation of adult oligodendrocyte precursor cells. Stem Cells 25:3204-3214.

Cheriyath V, Kuhns MA, Kalaycio ME, Borden EC (2011) Potentiation of apoptosis by histone deacetylase inhibitors and doxorubicin combination: cytoplasmic cathepsin B as a mediator of apoptosis in multiple myeloma. Br J Cancer 104:957-967.

Cheung WL, Briggs SD, Allis CD (2000) Acetylation and chromosomal functions. CurrOpinCell Biol 12:326-333. 
Chiurazzi P, Pomponi MG, Pietrobono R, Bakker CE, Neri G, Oostra BA (1999) Synergistic effect of histone hyperacetylation and DNA demethylation in the reactivation of the FMR1 gene. HumMolGenet 8:2317-2323.

Cimprich KA, Cortez D (2008) ATR: an essential regulator of genome integrity. Nat Rev Mol Cell Biol 9:616-627.

Colussi C, Illi B, Rosati J, Spallotta F, Farsetti A, Grasselli A, Mai A, Capogrossi MC, Gaetano C (2010) Histone deacetylase inhibitors: keeping momentum for neuromuscular and cardiovascular diseases treatment. Pharmacol Res 62:3-10.

Connor JR, Menzies SL (1996) Relationship of iron to oligodendrocytes and myelination. Glia 17:83-93.

Copray S, Huynh JL, Sher F, Casaccia-Bonnefil P, Boddeke E (2009) Epigenetic mechanisms facilitating oligodendrocyte development, maturation, and aging. Glia 57:1579-1587.

Covington HE, 3rd, Vialou VF, LaPlant Q, Ohnishi YN, Nestler EJ (2011) Hippocampal-dependent antidepressant-like activity of histone deacetylase inhibition. Neurosci Lett 493:122-126.

Dewar D, Underhill SM, Goldberg MP (2003) Oligodendrocytes and ischemic brain injury. J Cereb Blood Flow Metab 23:263-274.

Di Bernardo G, Squillaro T, Dell'Aversana C, Miceli M, Cipollaro M, Cascino A, Altucci L, Galderisi U (2009) Histone deacetylase inhibitors promote apoptosis and senescence in human mesenchymal stem cells. Stem Cells Dev 18:573-581.

Di Bernardo G, Alessio N, Dell'Aversana C, Casale F, Teti D, Cipollaro M, Altucci L, Galderisi U (2010) Impact of histone deacetylase inhibitors SAHA and MS-275 on DNA repair pathways in human mesenchymal stem cells. J Cell Physiol 225:537-544.

Dietrich J, Monje M, Wefel J, Meyers C (2008) Clinical patterns and biological correlates of cognitive dysfunction associated with cancer therapy. Oncologist 13:1285-1295.

Dietrich J, Han R, Yang Y, Mayer-Proschel M, Noble M (2006) CNS progenitor cells and oligodendrocytes are targets of chemotherapeutic agents in vitro and in vivo. J Biol 5:22.

Dietz KC, Casaccia P (2010) HDAC inhibitors and neurodegeneration: at the edge between protection and damage. Pharmacol Res 62:11-17. 
Dong G, Wang L, Wang CY, Yang T, Kumar MV, Dong Z (2008) Induction of apoptosis in renal tubular cells by histone deacetylase inhibitors, a family of anticancer agents. J Pharmacol Exp Ther 325:978-984.

Dugas JC, Tai YC, Speed TP, Ngai J, Barres BA (2006) Functional genomic analysis of oligodendrocyte differentiation. J Neurosci 26:10967-10983.

Dugas JC, Cuellar TL, Scholze A, Ason B, Ibrahim A, Emery B, Zamanian JL, Foo LC, McManus MT, Barres BA (2010) Dicer1 and miR-219 Are required for normal oligodendrocyte differentiation and myelination. Neuron 65:597-611.

Emery B (2010) Regulation of oligodendrocyte differentiation and myelination. Science 330:779-782.

Fancy SP, Kotter MR, Harrington EP, Huang JK, Zhao C, Rowitch DH, Franklin RJ (2010) Overcoming remyelination failure in multiple sclerosis and other myelin disorders. Exp Neurol 225:18-23.

Fennema-Notestine C, Archibald SL, Jacobson MW, Corey-Bloom J, Paulsen JS, Peavy GM, Gamst AC, Hamilton JM, Salmon DP, Jernigan TL (2004) In vivo evidence of cerebellar atrophy and cerebral white matter loss in Huntington disease. Neurology 63:989-995.

Ferguson RJ, Ahles TA (2003) Low neuropsychologic performance among adult cancer survivors treated with chemotherapy. Curr Neurol Neurosci Rep 3:215-222.

Ferreira AA, Pereira MJ, Manhaes AC, Barradas PC (2007) Ultrastructural identification of oligodendrocyte/myelin proteins in corpus callosum of hypothyroid animals. Int J Dev Neurosci 25:87-94.

Fields RD (2008) White matter in learning, cognition and psychiatric disorders. Trends Neurosci 31:361-370.

Fischer A, Sananbenesi F, Wang X, Dobbin M, Tsai LH (2007) Recovery of learning and memory is associated with chromatin remodelling. Nature 447:178-182.

Fischle W, Dequiedt F, Hendzel MJ, Guenther MG, Lazar MA, Voelter W, Verdin E (2002) Enzymatic activity associated with class II HDACs is dependent on a multiprotein complex containing HDAC3 and SMRT/N-CoR. MolCell 9:45-57.

Fishel ML, Vasko MR, Kelley MR (2007) DNA repair in neurons: so if they don't divide what's to repair? Mutat Res 614:24-36. 
Forgione N, Tropepe V (2011) Histone deacetylase inhibition promotes caspaseindependent cell death of ventral midbrain neurons. Mol Cell Neurosci.

Franco PG, Silvestroff L, Soto EF, Pasquini JM (2008) Thyroid hormones promote differentiation of oligodendrocyte progenitor cells and improve remyelination after cuprizone-induced demyelination. Exp Neurol 212:458467.

Frew AJ, Johnstone RW, Bolden JE (2009) Enhancing the apoptotic and therapeutic effects of HDAC inhibitors. Cancer Lett 280:125-133.

Fu H, Qi Y, Tan M, Cai J, Takebayashi H, Nakafuku M, Richardson W, Qiu M (2002) Dual origin of spinal oligodendrocyte progenitors and evidence for the cooperative role of Olig2 and Nkx2.2 in the control of oligodendrocyte differentiation. Development 129:681-693.

Gallo V, Zhou JM, McBain CJ, Wright P, Knutson PL, Armstrong RC (1996) Oligodendrocyte progenitor cell proliferation and lineage progression are regulated by glutamate receptor-mediated $\mathrm{K}+$ channel block. J Neurosci 16:2659-2670.

Genoud S, Lappe-Siefke C, Goebbels S, Radtke F, Aguet M, Scherer SS, Suter U, Nave KA, Mantei N (2002) Notch1 control of oligodendrocyte differentiation in the spinal cord. J Cell Biol 158:709-718.

Gilbert J, Baker SD, Bowling MK, Grochow L, Figg WD, Zabelina Y, Donehower RC, Carducci MA (2001) A phase I dose escalation and bioavailability study of oral sodium phenylbutyrate in patients with refractory solid tumor malignancies. Clin Cancer Res 7:2292-2300.

Givogri MI, Costa RM, Schonmann V, Silva AJ, Campagnoni AT, Bongarzone ER (2002) Central nervous system myelination in mice with deficient expression of Notch1 receptor. J Neurosci Res 67:309-320.

Gokhan S, Marin-Husstege M, Yung SY, Fontanez D, Casaccia-Bonnefil P, Mehler MF (2005) Combinatorial profiles of oligodendrocyte-selective classes of transcriptional regulators differentially modulate myelin basic protein gene expression. J Neurosci 25:8311-8321.

Gomes WA, Mehler MF, Kessler JA (2003) Transgenic overexpression of BMP4 increases astroglial and decreases oligodendroglial lineage commitment. Dev Biol 255:164-177.

Goodarzi AA, Noon AT, Deckbar D, Ziv Y, Shiloh Y, Lobrich M, Jeggo PA (2008) ATM signaling facilitates repair of DNA double-strand breaks associated with heterochromatin. Mol Cell 31:167-177. 
Grayson DR, Kundakovic M, Sharma RP (2010) Is there a future for histone deacetylase inhibitors in the pharmacotherapy of psychiatric disorders? Mol Pharmacol 77:126-135.

Grunstein M (1997) Histone acetylation in chromatin structure and transcription. Nature 389:349-352.

Gu W, Roeder RG (1997) Activation of p53 sequence-specific DNA binding by acetylation of the p53 C-terminal domain. Cell 90:595-606.

Haberland M, Montgomery RL, Olson EN (2009) The many roles of histone deacetylases in development and physiology: implications for disease and therapy. NatRevGenet 10:32-42.

Hahnen E, Hauke J, Trankle C, Eyupoglu IY, Wirth B, Blumcke I (2008) Histone deacetylase inhibitors: possible implications for neurodegenerative disorders. ExpertOpinInvestigDrugs 17:169-184.

Hahnen E, Eyupoglu IY, Brichta L, Haastert K, Trankle C, Siebzehnrubl FA, Riessland M, Holker I, Claus P, Romstock J, Buslei R, Wirth B, Blumcke I (2006) In vitro and ex vivo evaluation of second-generation histone deacetylase inhibitors for the treatment of spinal muscular atrophy. JNeurochem 98:193-202.

Hall ED, Springer JE (2004) Neuroprotection and acute spinal cord injury: a reappraisal. NeuroRx 1:80-100.

Hamanoue M, Matsuzaki Y, Sato K, Okano HJ, Shibata S, Sato I, Suzuki S, Ogawara M, Takamatsu K, Okano H (2009) Cell surface N-glycans mediated isolation of mouse neural stem cells. J Neurochem 110:15751584.

Han R, Yang YM, Dietrich J, Luebke A, Mayer-Proschel M, Noble M (2008) Systemic 5-fluorouracil treatment causes a syndrome of delayed myelin destruction in the central nervous system. J Biol 7:12.

Harikrishnan KN, Karagiannis TC, Chow MZ, El-Osta A (2008) Effect of valproic acid on radiation-induced DNA damage in euchromatic and heterochromatic compartments. Cell Cycle 7:468-476.

Harsan LA, Steibel J, Zaremba A, Agin A, Sapin R, Poulet P, Guignard B, Parizel N, Grucker D, Boehm N, Miller RH, Ghandour MS (2008) Recovery from chronic demyelination by thyroid hormone therapy: myelinogenesis induction and assessment by diffusion tensor magnetic resonance imaging. J Neurosci 28:14189-14201. 
Herman D, Jenssen K, Burnett R, Soragni E, Perlman SL, Gottesfeld JM (2006) Histone deacetylase inhibitors reverse gene silencing in Friedreich's ataxia. NatChemBiol 2:551-558.

Hill R, Lee PW (2010) The DNA-dependent protein kinase (DNA-PK): More than just a case of making ends meet? Cell Cycle 9:3460-3469.

Hirtz D, lannaccone S, Heemskerk J, Gwinn-Hardy K, Moxley R, III, Rowland LP (2005) Challenges and opportunities in clinical trials for spinal muscular atrophy. Neurology 65:1352-1357.

Hoshino M, Tagawa K, Okuda T, Murata M, Oyanagi K, Arai N, Mizutani T, Kanazawa I, Wanker EE, Okazawa $\mathrm{H}$ (2003) Histone deacetylase activity is retained in primary neurons expressing mutant huntingtin protein. JNeurochem 87:257-267.

Inagaki M, Yoshikawa E, Matsuoka Y, Sugawara Y, Nakano T, Akechi T, Wada $\mathrm{N}$, Imoto S, Murakami K, Uchitomi Y (2007) Smaller regional volumes of brain gray and white matter demonstrated in breast cancer survivors exposed to adjuvant chemotherapy. Cancer 109:146-156.

Insinga A, Monestiroli S, Ronzoni S, Gelmetti V, Marchesi F, Viale A, Altucci L, Nervi C, Minucci S, Pelicci PG (2005) Inhibitors of histone deacetylases induce tumor-selective apoptosis through activation of the death receptor pathway. Nat Med 11:71-76.

Ishibashi T, Dakin KA, Stevens B, Lee PR, Kozlov SV, Stewart CL, Fields RD (2006) Astrocytes promote myelination in response to electrical impulses. Neuron 49:823-832.

Itoh K, Stevens B, Schachner M, Fields RD (1995) Regulated expression of the neural cell adhesion molecule L1 by specific patterns of neural impulses. Science 270:1369-1372.

Jackson SP, Bartek J (2009) The DNA-damage response in human biology and disease. Nature 461:1071-1078.

Janelsins MC, Kohli S, Mohile SG, Usuki K, Ahles TA, Morrow GR (2011) An update on cancer- and chemotherapy-related cognitive dysfunction: current status. Semin Oncol 38:431-438.

Jansen CE, Dodd MJ, Miaskowski CA, Dowling GA, Kramer J (2008) Preliminary results of a longitudinal study of changes in cognitive function in breast cancer patients undergoing chemotherapy with doxorubicin and cyclophosphamide. Psychooncology 17:1189-1195. 
Jessen KR, Mirsky R (2008) Negative regulation of myelination: relevance for development, injury, and demyelinating disease. Glia 56:1552-1565.

Jones LL, Yamaguchi Y, Stallcup WB, Tuszynski MH (2002) NG2 is a major chondroitin sulfate proteoglycan produced after spinal cord injury and is expressed by macrophages and oligodendrocyte progenitors. J Neurosci 22:2792-2803.

Joshi S, Wang M, Etu JJ, Suckow RF, Cooper TB, Feinmark SJ, Bruce JN, Fine $R L$ (2008) Transient cerebral hypoperfusion enhances intraarterial carmustine deposition into brain tissue. J Neurooncol 86:123-132.

Kao GD, McKenna WG, Guenther MG, Muschel RJ, Lazar MA, Yen TJ (2003) Histone deacetylase 4 interacts with 53BP1 to mediate the DNA damage response. J Cell Biol 160:1017-1027.

Karadottir R, Hamilton NB, Bakiri Y, Attwell D (2008) Spiking and nonspiking classes of oligodendrocyte precursor glia in CNS white matter. Nat Neurosci 11:450-456.

Karagiannis TC, Harikrishnan KN, El-Osta A (2007) Disparity of histone deacetylase inhibition on repair of radiation-induced DNA damage on euchromatin and constitutive heterochromatin compartments. Oncogene 26:3963-3971.

Kaya E, Keskin L, Aydogdu I, Kuku I, Bayraktar N, Erkut MA (2005) Oxidant/antioxidant parameters and their relationship with chemotherapy in Hodgkin's lymphoma. J Int Med Res 33:687-692.

Kazantsev AG, Thompson LM (2008) Therapeutic application of histone deacetylase inhibitors for central nervous system disorders. NatRevDrug Discov 7:854-868.

Kelly WK, Richon VM, O'Connor O, Curley T, MacGregor-Curtelli B, Tong W, Klang M, Schwartz L, Richardson S, Rosa E, Drobnjak M, Cordon-Cordo C, Chiao JH, Rifkind R, Marks PA, Scher H (2003) Phase I clinical trial of histone deacetylase inhibitor: suberoylanilide hydroxamic acid administered intravenously. Clin Cancer Res 9:3578-3588.

Kelly WK, O'Connor OA, Krug LM, Chiao JH, Heaney M, Curley T, MacGregoreCortelli B, Tong W, Secrist JP, Schwartz L, Richardson S, Chu E, Olgac S, Marks PA, Scher H, Richon VM (2005) Phase I study of an oral histone deacetylase inhibitor, suberoylanilide hydroxamic acid, in patients with advanced cancer. J Clin Oncol 23:3923-3931. 
Kernochan LE, Russo ML, Woodling NS, Huynh TN, Avila AM, Fischbeck KH, Sumner CJ (2005) The role of histone acetylation in SMN gene expression. HumMolGenet 14:1171-1182.

Kidd SK, Schneider JS (2010) Protection of dopaminergic cells from MPP+mediated toxicity by histone deacetylase inhibition. Brain Res 1354:172178.

Kim MS, Blake M, Baek JH, Kohlhagen G, Pommier Y, Carrier F (2003) Inhibition of histone deacetylase increases cytotoxicity to anticancer drugs targeting DNA. Cancer Res 63:7291-7300.

Knobler RL, Stempak JG, Laurencin M (1974) Oligodendroglial ensheathment of axons during myelination in the developing rat central nervous system. A serial section electron microscopical sttudy. JUltrastructRes 49:34-49.

Konat GW, Kraszpulski M, James I, Zhang HT, Abraham J (2008) Cognitive dysfunction induced by chronic administration of common cancer chemotherapeutics in rats. Metab Brain Dis 23:325-333.

Kondo T, Raff M (2000) The Id4 HLH protein and the timing of oligodendrocyte differentiation. EMBO J 19:1998-2007.

Kremer D, Aktas O, Hartung HP, Kury P (2011) The complex world of oligodendroglial differentiation inhibitors. Ann Neurol 69:602-618.

Ladiwala U, Li H, Antel JP, Nalbantoglu J (1999) p53 induction by tumor necrosis factor-alpha and involvement of p53 in cell death of human oligodendrocytes. J Neurochem 73:605-611.

Lane AA, Chabner BA (2009) Histone deacetylase inhibitors in cancer therapy. J Clin Oncol 27:5459-5468.

Larsen PH, Yong VW (2004) The expression of matrix metalloproteinase-12 by oligodendrocytes regulates their maturation and morphological differentiation. J Neurosci 24:7597-7603.

Ledoux SP, Shen CC, Grishko VI, Fields PA, Gard AL, Wilson GL (1998) Glial cell-specific differences in response to alkylation damage. Glia 24:304312.

LeDoux SP, Williams BA, Hollensworth BS, Shen C, Thomale J, Rajewsky MF, Brent TP, Wilson GL (1996) Glial cell-specific differences in repair of O6methylguanine. Cancer Res 56:5615-5619. 
Lee J, Wu Y, Qi Y, Xue H, Liu Y, Scheel D, German M, Qiu M, Guillemot F, Rao M, Gradwohl G (2003) Neurogenin3 participates in gliogenesis in the developing vertebrate spinal cord. Dev Biol 253:84-98.

Lee JH, Choy ML, Ngo L, Foster SS, Marks PA (2010) Histone deacetylase inhibitor induces DNA damage, which normal but not transformed cells can repair. Proc Natl Acad Sci U S A 107:14639-14644.

Lee S, Park JR, Seo MS, Roh KH, Park SB, Hwang JW, Sun B, Seo K, Lee YS, Kang SK, Jung JW, Kang KS (2009) Histone deacetylase inhibitors decrease proliferation potential and multilineage differentiation capability of human mesenchymal stem cells. Cell Prolif 42:711-720.

Li H, Richardson WD (2009) Genetics meets epigenetics: HDACs and Wnt signaling in myelin development and regeneration. NatNeurosci 12:815817.

Lieber MR (2008) The mechanism of human nonhomologous DNA end joining. J Biol Chem 283:1-5.

Ligon KL, Kesari S, Kitada M, Sun T, Arnett HA, Alberta JA, Anderson DJ, Stiles CD, Rowitch DH (2006) Development of NG2 neural progenitor cells requires Olig gene function. Proc Natl Acad Sci U S A 103:7853-7858.

Lin T, Xiang Z, Cui L, Stallcup W, Reeves SA (2006) New mouse oligodendrocyte precursor (mOP) cells for studies on oligodendrocyte maturation and function. J Neurosci Methods 157:187-194.

Liu A, Li J, Marin-Husstege M, Kageyama R, Fan Y, Gelinas C, CasacciaBonnefil P (2006) A molecular insight of Hes5-dependent inhibition of myelin gene expression: old partners and new players. EMBO J 25:48334842.

Liu A, Han YR, Li J, Sun D, Ouyang M, Plummer MR, Casaccia-Bonnefil P (2007a) The glial or neuronal fate choice of oligodendrocyte progenitors is modulated by their ability to acquire an epigenetic memory. JNeurosci 27:7339-7343.

Liu H, Hu Q, D'Ercole AJ, Ye P (2009) Histone deacetylase 11 regulates oligodendrocyte-specific gene expression and cell development in OL-1 oligodendroglia cells. Glia 57:1-12.

Liu Z, Hu X, Cai J, Liu B, Peng X, Wegner M, Qiu M (2007b) Induction of oligodendrocyte differentiation by Olig2 and Sox10: evidence for reciprocal interactions and dosage-dependent mechanisms. Dev Biol 302:683-693. 
Lombard DB, Chua KF, Mostoslavsky R, Franco S, Gostissa M, Alt FW (2005) DNA repair, genome stability, and aging. Cell 120:497-512.

Lyssiotis CA, Walker J, Wu C, Kondo T, Schultz PG, Wu X (2007) Inhibition of histone deacetylase activity induces developmental plasticity in oligodendrocyte precursor cells. ProcNatIAcadSciUSA 104:14982-14987.

Lytle JM, Chittajallu R, Wrathall JR, Gallo V (2009) NG2 cell response in the CNP-EGFP mouse after contusive spinal cord injury. Glia 57:270-285.

Mabie PC, Mehler MF, Kessler JA (1999) Multiple roles of bone morphogenetic protein signaling in the regulation of cortical cell number and phenotype. $J$ Neurosci 19:7077-7088.

Mangin JM, Gallo V (2011) The curious case of NG2 cells: transient trend or game changer? ASN Neuro 3.

Marchion DC, Bicaku E, Daud Al, Richon V, Sullivan DM, Munster PN (2004) Sequence-specific potentiation of topoisomerase II inhibitors by the histone deacetylase inhibitor suberoylanilide hydroxamic acid. J Cell Biochem 92:223-237.

Marin-Husstege M, Muggironi M, Liu A, Casaccia-Bonnefil P (2002) Histone deacetylase activity is necessary for oligodendrocyte lineage progression. JNeurosci 22:10333-10345.

Marks PA (2007) Discovery and development of SAHA as an anticancer agent. Oncogene 26:1351-1356.

Marks PA (2010) The clinical development of histone deacetylase inhibitors as targeted anticancer drugs. Expert Opin Investig Drugs 19:1049-1066.

Marshall JL, Rizvi N, Kauh J, Dahut W, Figuera M, Kang MH, Figg WD, Wainer I, Chaissang C, Li MZ, Hawkins MJ (2002) A phase I trial of depsipeptide (FR901228) in patients with advanced cancer. J Exp Ther Oncol 2:325332.

McGee-Lawrence ME, McCleary-Wheeler AL, Secreto FJ, Razidlo DF, Zhang M, Stensgard BA, Li X, Stein GS, Lian JB, Westendorf JJ (2011) Suberoylanilide hydroxamic acid (SAHA; vorinostat) causes bone loss by inhibiting immature osteoblasts. Bone 48:1117-1126.

McIntosh AM, Hall J, Lymer GK, Sussmann JE, Lawrie SM (2009) Genetic risk for white matter abnormalities in bipolar disorder. Int Rev Psychiatry 21:387-393. 
McTigue DM, Tripathi RB (2008) The life, death, and replacement of oligodendrocytes in the adult CNS. J Neurochem 107:1-19.

Meattini I, Scotti V, Pescini F, Livi L, Sulprizio S, Palumbo V, Sarti C, Biti G (2010) Ischemic stroke during cisplatin-based chemotherapy for testicular germ cell tumor: case report and review of the literature. J Chemother 22:134-136.

Meyers CA (2008) How chemotherapy damages the central nervous system. J Biol 7:11.

Miller KM, Tjeertes JV, Coates J, Legube G, Polo SE, Britton S, Jackson SP (2010) Human HDAC1 and HDAC2 function in the DNA-damage response to promote DNA nonhomologous end-joining. Nat Struct Mol Biol 17:11441151.

Miller RH, Mi S (2007) Dissecting demyelination. Nat Neurosci 10:1351-1354.

Misteli T, Soutoglou E (2009) The emerging role of nuclear architecture in DNA repair and genome maintenance. Nat Rev Mol Cell Biol 10:243-254.

Mock DJ, Strathmann F, Blumberg BM, Mayer-Proschel M (2006) Infection of murine oligodendroglial precursor cells with Human Herpesvirus 6 (HHV6)--establishment of a murine in vitro model. J Clin Virol 37 Suppl 1:S1723.

Mok TS, Lam KC, Lee C, Zhang L, Wong H, Chan AT, Yeo W, Yim AP, Chak K, Zee $B(2005)$ Phase II randomized study comparing the toxicity profile of gemcitabine plus cisplatin with gemcitabine plus oral etoposide in the treatment of advanced non-small cell lung cancer. Oncology 68:485-492.

Monje M (2008) Cranial radiation therapy and damage to hippocampal neurogenesis. Dev Disabil Res Rev 14:238-242.

Mouzannar R, Miric SJ, Wiggins RC, Konat GW (2001) Hydrogen peroxide induces rapid digestion of oligodendrocyte chromatin into high molecular weight fragments. Neurochem Int 38:9-15.

Nadin SB, Vargas-Roig LM, Drago G, Ibarra J, Ciocca DR (2006) DNA damage and repair in peripheral blood lymphocytes from healthy individuals and cancer patients: a pilot study on the implications in the clinical response to chemotherapy. Cancer Lett 239:84-97.

Nave KA (2010) Myelination and support of axonal integrity by glia. Nature 468:244-252. 
Nebbioso A, Clarke N, Voltz E, Germain E, Ambrosino C, Bontempo P, Alvarez $R$, Schiavone EM, Ferrara F, Bresciani F, Weisz A, de Lera AR, Gronemeyer H, Altucci L (2005) Tumor-selective action of HDAC inhibitors involves TRAIL induction in acute myeloid leukemia cells. Nat Med 11:7784.

Nicolay DJ, Doucette JR, Nazarali AJ (2007) Transcriptional control of oligodendrogenesis. Glia 55:1287-1299.

Nishiyama A (1998) Glial progenitor cells in normàl and pathological states. Keio J Med 47:205-208.

Nishiyama A, Komitova M, Suzuki R, Zhu X (2009) Polydendrocytes (NG2 cells): multifunctional cells with lineage plasticity. Nat Rev Neurosci 10:9-22.

Nissen-Meyer LS, Svalheim S, Tauboll E, Reppe S, Lekva T, Solberg LB, Melhus G, Reinholt FP, Gjerstad L, Jemtland R (2007) Levetiracetam, phenytoin, and valproate act differently on rat bone mass, structure, and metabolism. Epilepsia 48:1850-1860.

Noble M, Wren D, Wolswijk G (1992) The O-2A(adult) progenitor cell: a glial stem cell of the adult central nervous system. Semin Cell Biol 3:413-422.

Ohri SS, Maddie MA, Zhao Y, Qiu MS, Hetman M, Whittemore SR (2011) Attenuating the endoplasmic reticulum stress response improves functional recovery after spinal cord injury. Glia 59:1489-1502.

Papageorgiou M, Stiakaki E, Dimitriou H, Malliaraki N, Notas G, Castanas E, Kalmanti M (2005) Cancer chemotherapy reduces plasma total antioxidant capacity in children with malignancies. Leuk Res 29:11-16.

Park SK, Solomon D, Vartanian T (2001) Growth factor control of CNS myelination. Dev Neurosci 23:327-337.

Pedre X, Mastronardi F, Bruck W, Lopez-Rodas G, Kuhlmann T, Casaccia P (2011) Changed histone acetylation patterns in normal-appearing white matter and early multiple sclerosis lesions. J Neurosci 31:3435-3445.

Pietrzak M, Smith SC, Geralds JT, Hagg T, Gomes C, Hetman M (2011) Nucleolar disruption and apoptosis are distinct neuronal responses to etoposide-induced DNA damage. J Neurochem 117:1033-1046.

Plant GW, Currier PF, Cuervo EP, Bates ML, Pressman Y, Bunge MB, Wood PM (2002) Purified adult ensheathing glia fail to myelinate axons under culture conditions that enable Schwann cells to form myelin. JNeurosci 22:60836091. 
Pratap J, Akech J, Wixted JJ, Szabo G, Hussain S, McGee-Lawrence ME, Li X, Bedard K, Dhillon RJ, van Wijnen AJ, Stein JL, Stein GS, Westendorf JJ, Lian JB (2010) The histone deacetylase inhibitor, vorinostat, reduces tumor growth at the metastatic bone site and associated osteolysis, but promotes normal bone loss. Mol Cancer Ther 9:3210-3220.

Pringproa K, Kumnok J, Ulrich R, Baumgartner W, Wewetzer K (2008) In vitro characterization of a murine oligodendrocyte precursor cell line (BO-1) following spontaneous immortalization. Int J Dev Neurosci 26:283-291.

Purrucker JC, Fricke A, Ong MF, Rube C, Rube CE, Mahlknecht U (2010) HDAC inhibition radiosensitizes human normal tissue cells and reduces DNA Double-Strand Break repair capacity. Oncol Rep 23:263-269.

Qi Y, Cai J, Wu Y, Wu R, Lee J, Fu H, Rao M, Sussel L, Rubenstein J, Qiu M (2001) Control of oligodendrocyte differentiation by the Nkx2.2 homeodomain transcription factor. Development 128:2723-2733.

Richon VM, Sandhoff TW, Rifkind RA, Marks PA (2000) Histone deacetylase inhibitor selectively induces P21WAF1 expression and gene-associated histone acetylation. Proc Natl Acad Sci U S A 97:10014-10019.

Robert T, Vanoli F, Chiolo I, Shubassi G, Bernstein KA, Rothstein R, Botrugno OA, Parazzoli D, Oldani A, Minucci S, Foiani M (2011) HDACs link the DNA damage response, processing of double-strand breaks and autophagy. Nature 471:74-79.

Roth AD, Ramirez G, Alarcon R, Von Bernhardi R (2005) Oligodendrocytes damage in Alzheimer's disease: beta amyloid toxicity and inflammation. Biol Res 38:381-387.

Ryan QC, Headlee D, Acharya M, Sparreboom A, Trepel JB, Ye J, Figg WD, Hwang K, Chung EJ, Murgo A, Melillo G, Elsayed Y, Monga M, Kalnitskiy M, Zwiebel J, Sausville EA (2005) Phase I and pharmacokinetic study of MS-275, a histone deacetylase inhibitor, in patients with advanced and refractory solid tumors or lymphoma. J Clin Oncol 23:3912-3922.

Rzeski W, Pruskil S, Macke A, Felderhoff-Mueser U, Reiher AK, Hoerster F, Jansma C, Jarosz B, Stefovska V, Bittigau P, Ikonomidou C (2004) Anticancer agents are potent neurotoxins in vitro and in vivo. Ann Neurol $56: 351-360$.

Sahni V, Kessler JA (2010) Stem cell therapies for spinal cord injury. Nat Rev Neurol 6:363-372. 
Samanta J, Kessler JA (2004) Interactions between ID and OLIG proteins mediate the inhibitory effects of BMP4 on oligodendroglial differentiation. Development 131:4131-4142.

San Filippo J, Sung P, Klein H (2008) Mechanism of eukaryotic homologous recombination. Annu Rev Biochem 77:229-257.

Sandor V, Senderowicz A, Mertins S, Sackett D, Sausville E, Blagosklonny MV, Bates SE (2000) P21-dependent g(1)arrest with downregulation of cyclin D1 and upregulation of cyclin $E$ by the histone deacetylase inhibitor FR901228. Br J Cancer 83:817-825.

Sandor V, Bakke S, Robey RW, Kang MH, Blagosklonny MV, Bender J, Brooks R, Piekarz RL, Tucker E, Figg WD, Chan KK, Goldspiel B, Fojo AT, Balcerzak SP, Bates SE (2002) Phase I trial of the histone deacetylase inhibitor, depsipeptide (FR901228, NSC 630176), in patients with refractory neoplasms. Clin Cancer Res 8:718-728.

Saykin AJ, Ahles TA, McDonald BC (2003) Mechanisms of chemotherapyinduced cognitive disorders: neuropsychological, pathophysiological, and neuroimaging perspectives. Semin Clin Neuropsychiatry 8:201-216.

Schroeder FA, Lin CL, Crusio WE, Akbarian S (2007) Antidepressant-like effects of the histone deacetylase inhibitor, sodium butyrate, in the mouse. BiolPsychiatry 62:55-64.

Seigers R, Fardell JE (2011) Neurobiological basis of chemotherapy-induced cognitive impairment: a review of rodent research. Neurosci Biobehav Rev 35:729-741.

Seigers R, Schagen SB, Coppens CM, van der Most PJ, van Dam FS, Koolhaas JM, Buwalda B (2009) Methotrexate decreases hippocampal cell proliferation and induces memory deficits in rats. Behav Brain Res 201:279-284.

Seigers R, Schagen SB, Beerling W, Boogerd W, van Tellingen O, van Dam FS, Koolhaas JM, Buwalda B (2008) Long-lasting suppression of hippocampal cell proliferation and impaired cognitive performance by methotrexate in the rat. Behav Brain Res 186:168-175.

Shahbazian MD, Grunstein M (2007) Functions of site-specific histone acetylation and deacetylation. AnnuRevBiochem 76:75-100.

Shen S, Casaccia-Bonnefil P (2008) Post-translational modifications of nucleosomal histones in oligodendrocyte lineage cells in development and disease. JMolNeurosci 35:13-22. 
Shen S, Li J, Casaccia-Bonnefil P (2005) Histone modifications affect timing of oligodendrocyte progenitor differentiation in the developing rat brain. JCell Biol 169:577-589.

Shen S, Sandoval J, Swiss VA, Li J, Dupree J, Franklin RJ, Casaccia-Bonnefil P (2008) Age-dependent epigenetic control of differentiation inhibitors is critical for remyelination efficiency. NatNeurosci 11:1024-1034.

Sheth RD, Wesolowski CA, Jacob JC, Penney S, Hobbs GR, Riggs JE, Bodensteiner JB (1995) Effect of carbamazepine and valproate on bone mineral density. J Pediatr 127:256-262.

Shiozawa K, Nakanishi T, Tan M, Fang HB, Wang WC, Edelman MJ, Carlton D, Gojo I, Sausville EA, Ross DD (2009) Preclinical studies of vorinostat (suberoylanilide hydroxamic acid) combined with cytosine arabinoside and etoposide for treatment of acute leukemias. Clin Cancer Res 15:16981707.

Smith GC, Jackson SP (1999) The DNA-dependent protein kinase. Genes Dev 13:916-934.

Southwood C, He C, Garbern J, Kamholz J, Arroyo E, Gow A (2004) CNS myelin paranodes require Nkx6-2 homeoprotein transcriptional activity for normal structure. J Neurosci 24:11215-11225.

Stemmer SM, Stears JC, Burton BS, Jones RB, Simon JH (1994) White matter changes in patients with breast cancer treated with high-dose chemotherapy and autologous bone marrow support. AJNR Am J Neuroradiol 15:1267-1273.

Takahashi N, Sakurai T, Davis KL, Buxbaum JD (2011) Linking oligodendrocyte and myelin dysfunction to neurocircuitry abnormalities in schizophrenia. Prog Neurobiol 93:13-24.

Talbott JF, Loy DN, Liu Y, Qiu MS, Bunge MB, Rao MS, Whittemore SR (2005) Endogenous Nkx2.2+/Olig2+ oligodendrocyte precursor cells fail to remyelinate the demyelinated adult rat spinal cord in the absence of astrocytes. ExpNeurol 192:11-24.

Talbott JF, Cao Q, Enzmann GU, Benton RL, Achim V, Cheng XX, Mills MD, Rao MS, Whittemore SR (2006) Schwann cell-like differentiation by adult oligodendrocyte precursor cells following engraftment into the demyelinated spinal cord is BMP-dependent. Glia 54:147-159. 
Tannock IF, Ahles TA, Ganz PA, Van Dam FS (2004) Cognitive impairment associated with chemotherapy for cancer: report of a workshop. J Clin Oncol 22:2233-2239.

Thomas EA (2009) Focal nature of neurological disorders necessitates isotypeselective histone deacetylase (HDAC) inhibitors. Mol Neurobiol 40:33-45.

Thorburne SK, Juurlink BH (1996) Low glutathione and high iron govern the susceptibility of oligodendroglial precursors to oxidative stress. J Neurochem 67:1014-1022.

Thuret S, Moon LD, Gage FH (2006) Therapeutic interventions after spinal cord injury. NatRevNeurosci 7:628-643.

Tjeertes JV, Miller KM, Jackson SP (2009) Screen for DNA-damage-responsive histone modifications identifies H3K9Ac and H3K56Ac in human cells. EMBO J 28:1878-1889.

Totoiu MO, Keirstead HS (2005) Spinal cord injury is accompanied by chronic progressive demyelination. JComp Neurol 486:373-383.

Trotter J, Karram K, Nishiyama A (2010) NG2 cells: Properties, progeny and origin. Brain Res Rev 63:72-82.

Tsankova N, Renthal W, Kumar A, Nestler EJ (2007) Epigenetic regulation in psychiatric disorders. NatRevNeurosci 8:355-367.

Tsankova NM, Berton O, Renthal W, Kumar A, Neve RL, Nestler EJ (2006) Sustained hippocampal chromatin regulation in a mouse model of depression and antidepressant action. NatNeurosci 9:519-525.

Uberti D, Yavin E, Gil S, Ayasola KR, Goldfinger N, Rotter V (1999) Hydrogen peroxide induces nuclear translocation of p53 and apoptosis in cells of oligodendroglia origin. Brain Res Mol Brain Res 65:167-175.

Ullen $F(2009)$ Is activity regulation of late myelination a plastic mechanism in the human nervous system? Neuron Glia Biol 5:29-34.

Ungerstedt JS, Sowa Y, Xu WS, Shao Y, Dokmanovic M, Perez G, Ngo L, Holmgren A, Jiang X, Marks PA (2005) Role of thioredoxin in the response of normal and transformed cells to histone deacetylase inhibitors. Proc Natl Acad Sci U S A 102:673-678.

Vega RB, Matsuda K, Oh J, Barbosa AC, Yang X, Meadows E, McAnally J, Pomajzl C, Shelton JM, Richardson JA, Karsenty G, Olson EN (2004) 
Histone deacetylase 4 controls chondrocyte hypertrophy during skeletogenesis. Cell 119:555-566.

Wang S, Sdrulla A, Johnson JE, Yokota Y, Barres BA (2001) A role for the helixloop-helix protein Id2 in the control of oligodendrocyte development. Neuron 29:603-614.

Wang S, Sdrulla AD, diSibio G, Bush G, Nofziger D, Hicks C, Weinmaster G, Barres BA (1998) Notch receptor activation inhibits oligodendrocyte differentiation. Neuron 21:63-75.

Wang Y, Cheng X, He Q, Zheng Y, Kim DH, Whittemore SR, Cao QL (2011) Astrocytes from the contused spinal cord inhibit oligodendrocyte differentiation of adult oligodendrocyte precursor cells by increasing the expression of bone morphogenetic proteins. J Neurosci 31:6053-6058.

Wenning GK, Stefanova N, Jellinger KA, Poewe W, Schlossmacher MG (2008) Multiple system atrophy: a primary oligodendrogliopathy. Ann Neurol 64:239-246.

Whittemore SR, Morassutti DJ, Walters WM, Liu RH, Magnuson DS (1999) Mitogen and substrate differentially affect the lineage restriction of adult rat subventricular zone neural precursor cell populations. Exp Cell Res 252:75-95.

Wick A, Wick W, Hirrlinger J, Gerhardt E, Dringen R, Dichgans J, Weller M, Schulz JB (2004) Chemotherapy-induced cell death in primary cerebellar granule neurons but not in astrocytes: in vitro paradigm of differential neurotoxicity. J Neurochem 91:1067-1074.

Wolswijk G, Noble M (1989) Identification of an adult-specific glial progenitor cell. Development 105:387-400.

Wosik K, Antel J, Kuhlmann T, Bruck W, Massie B, Nalbantoglu J (2003) Oligodendrocyte injury in multiple sclerosis: a role for p53. J Neurochem 85:635-644.

Wu Y, Liu Y, Levine EM, Rao MS (2003) Hes1 but not Hes5 regulates an astrocyte versus oligodendrocyte fate choice in glial restricted precursors. Dev Dyn 226:675-689.

Xu J, Chen S, Ahmed SH, Chen H, Ku G, Goldberg MP, Hsu CY (2001) Amyloidbeta peptides are cytotoxic to oligodendrocytes. J Neurosci 21:RC118.

Yang M, Kim JS, Song MS, Kim SH, Kang SS, Bae CS, Kim JC, Wang H, Shin T, Moon C (2010) Cyclophosphamide impairs hippocampus-dependent 
learning and memory in adult mice: Possible involvement of hippocampal neurogenesis in chemotherapy-induced memory deficits. Neurobiol Learn Mem 93:487-494.

Yang XJ, Seto E (2003) Collaborative spirit of histone deacetylases in regulating chromatin structure and gene expression. CurrOpinGenetDev 13:143-153.

Ye F, Chen Y, Hoang T, Montgomery RL, Zhao XH, Bu H, Hu T, Taketo MM, van Es JH, Clevers H, Hsieh J, Bassel-Duby R, Olson EN, Lu QR (2009) HDAC1 and HDAC2 regulate oligodendrocyte differentiation by disrupting the beta-catenin-TCF interaction. NatNeurosci 12:829-838.

Yu Y, Casaccia P, Lu QR (2010) Shaping the oligodendrocyte identity by epigenetic control. Epigenetics 5:124-128.

Yuan X, Chittajallu R, Belachew S, Anderson S, McBain CJ, Gallo V (2002) Expression of the green fluorescent protein in the oligodendrocyte lineage: a transgenic mouse for developmental and physiological studies. JNeurosciRes 70:529-545.

Zhang CL, McKinsey TA, Chang S, Antos CL, Hill JA, Olson EN (2002) Class II histone deacetylases act as signal-responsive repressors of cardiac hypertrophy. Cell 110:479-488.

Zhang Y, Li N, Caron C, Matthias G, Hess D, Khochbin S, Matthias P (2003) HDAC-6 interacts with and deacetylates tubulin and microtubules in vivo. EMBO J 22:1168-1179.

Zhang Y, Kwon S, Yamaguchi T, Cubizolles F, Rousseaux S, Kneissel M, Cao C, Li N, Cheng HL, Chua K, Lombard D, Mizeracki A, Matthias G, Alt FW, Khochbin S, Matthias P (2008) Mice lacking histone deacetylase 6 have hyperacetylated tubulin but are viable and develop normally. MolCell Biol 28:1688-1701.

Zhang ZG, Chopp M (2009) Neurorestorative therapies for stroke: underlying mechanisms and translation to the clinic. Lancet Neurol 8:491-500.

Zhao X, He X, Han X, Yu Y, Ye F, Chen Y, Hoang T, Xu X, Mi QS, Xin M, Wang F, Appel B, Lu QR (2010) MicroRNA-mediated control of oligodendrocyte differentiation. Neuron 65:612-626.

Zhou Q, Anderson DJ (2002) The bHLH transcription factors OLIG2 and OLIG1 couple neuronal and glial subtype specification. Cell 109:61-73. 
Ziskin JL, Nishiyama A, Rubio M, Fukaya M, Bergles DE (2007) Vesicular release of glutamate from unmyelinated axons in white matter. Nat Neurosci 10:321-330. 


\section{CURRICULUM VITAE}

NAME: $\quad$ Toros Ali Dincman

ADDRESS: Department of Anatomical Sciences and Neurobiology University of Louisville, School of Medicine

500 S. Preston St., Room 916A

Louisville, KY 40202

DOB: $\quad$ Miami, Florida- January 1, 1982

EDUCATION

\& TRAINING: $\quad$ A.B. with Honors, Biological Sciences University of Chicago

2000-04

MD/PhD Dual Degree Program

University of Louisville School of Medicine 2006-Present

\section{PUBLICATIONS:}

Strauss B., Kelly K., Dincman T., Ekiert D., Biesieda T., Song R. Cell death in Escherichia coli dnaE(Ts) mutants incubated at a nonpermissive temperature is prevented by mutation in the cydA gene. J Bacteriol. 2004 Apr;186:2147-55.

Zhang Y.P., Burke D.A., Shields L.B., Chekmenev S.Y., Dincman T., Zhang Y., Zheng Y., Smith R.R., Benton R.L, DeVries W.H., Hu X., Magnuson D.S., Whittemore S.R., Shields C.B. Spinal cord contusion based on precise vertebral stabilization and tissue displacement measured by combined assessment to discriminate small functional differences. J Neurotrauma. 2008 Oct;25:1227-40.

Benton R.L., Maddie M.A., Dincman T.A., Hagg T., Whittemore S.R. Transcriptional activation of endothelial cells by TGF $\beta$ coincides with acute microvascular plasticity following focal spinal cord ischemia- reperfusion injury. ASN Neuro. 2009 Aug 26;1(3). pii: e00015. doi: 10.1042/AN20090008.

Carasquillo M.M., Belbin O., Zou F., Allen M., Ertekin-Taner N., Ansari M., Wilcox S.L., Kashino M.R., Ma L., Younkin L.H., Younkin S.G., Younkin C.S., Dincman T.A., Howard M.E., Howell C.C., Stanton C.M., Watson C.M., Crump M., Vitart V., Hayward C., Hastie N.D., Rudan I., Campbell H., Polasek O., Brown K., 
Passmore P., Craig D., McGuiness B., Todd S., Kehoe P.G., Mann D.M., Smith A.D., Beaumont H., Warden D., Homes C., Heun R., Kölsch H., Kalsheker N., Pankratz V.S., Dickson D.W., Graff-Radford N.R., Petersen R.C., Wright A.F., Younkin S.G., Morgan K. Concordent Association of insulin degrading enzyme gene (IDE) variants with IDE mRNA, A $\beta$, and Alzheimer's disease. Submitted to Plos One.

Dincman T.A., Beare J.E., Ohri S.S., Whittemore S.R. Isolation of cortically derived mouse oligodendrocyte precursor cells. Submitted.

Dincman, T.A., Beare J.E., Gallo V., Hetman M., Whittemore S.R. Histone deacetylase inhibition mediated cytotoxicity of developing oligodendrocytes. In Preparation.

POSTERS:

Dincman T., Benton R.L., Maddie M., Merchant M., Whittemore S.R. 2007. Transcriptomic and Proteomic Analysis of Spinal Microvascular Endothelial Plasticity Following Focal Ischemic Spinal Cord Injury. The $26^{\text {th }}$ Annual National Neurotrauma Symposium, Orlando, FL.

Dincman T.A., Saraswat S., Whittemore S.R. 2009. Dysregulation of Chromatin Modifying Enzymes Following Contusive Spinal Cord Injury in the Adult Mouse. The $15^{\text {th }}$ Annual Kentucky Spinal Cord and Head Injury Research Trust (KSCHIRT) Symposium, Louisville, KY.

Dincman T.A., Beare J.E., Ohri S.S., Kuypers N.J., Gallo V., Whittemore S.R. 2010. Histone Deacetylase Responses to Contusive Spinal Cord Injury and ER Stress in Mouse Oligodendrocyte Precursor Cells. The $40^{\text {th }}$ Annual Neuroscience Meeting, San Diego, CA.

Dincman T.A., Beare J.E., Ohri S.S., Yaddanapuddi K., Gallo V., Whittemore S.R. 2011. Histone Deacetylase Inhibition Mediated Cytotoxicity of Oligodendrocyte Precursor Cells. Neuroscience Day, Louisville, KY.

Dincman T.A., Beare J.E., Ohri S.S., Gallo V., Hetman M., Whittemore S.R. 2011. Histone Deacetylase Inhibition Mediated Cytotoxicity of Oligodendrocyte Precursor Cells. The $41^{\text {st }}$ Annual Neuroscience Meeting, Washington D.C.

AWARDS: First Place Award Winner for Poster Presentation, Research! Louisville, Professional Students; $2^{\text {nd }}-4^{\text {th }}$ Year Medical Student Category, University of Louisville, 2007

Summer Research Scholars Program Award Recipient, University of Louisville School of Medicine, Kentucky Spinal Cord Injury Research Center, Dr. Richard Benton, 06/2007-08/2007 
Summer Research Scholars Program Award Recipient, University of Louisville School of Medicine, Kentucky Spinal Cord Injury Research Center, Dr. Christopher Shields, 06/2006-08/2006

MD/PhD Scholarship, University of Louisville, 2006- Present

A.B. in Biological Sciences (Cellular and Molecular Biology Specialization) with Honors, 2004

Dean's List, University of Chicago, $2001-2004$

Robert B. and Joseph Meyer Scholarship, University of Chicago, $2000-2004$

Summer Undergraduate Research Fellow (SURF), Mayo Clinic, Dr. Steven G. Younkin, 07/2003-09/2003

PROFESSIONAL SOCIETIES:

\author{
American Medical Association (AMA) \\ American Medical Student Association (AMSA) \\ Society for Neuroscience \\ Sigma Xi
}

